\title{
Engineering Bacillus subtilis for the formation of a durable living biocomposite material
}

Sun-Young Kang ${ }^{1,3^{*}}$, Anaya Pokhrel ${ }^{1,3^{*}}$, Sara Bratsch ${ }^{1,3^{*}}$, Joey J. Benson ${ }^{2}$, Seung-Oh Seo ${ }^{1,3}$, Maureen B. Quin ${ }^{1,3}$, Alptekin Aksan ${ }^{2,3}$, Claudia Schmidt-Dannert ${ }^{1,3^{* *}}$

1 Department of Biochemistry, Molecular Biology \& Biochemistry, University of Minnesota, Minneapolis, MN 55455, USA

${ }^{2}$ Department of Mechanical Engineering, University of Minnesota, Minneapolis, MN 55455, USA

${ }^{3}$ BioTechnology Institute, University of Minnesota, St. Paul, MN 55108, USA

* These authors contributed equally to this work.

\section{${ }^{\star *}$ Corresponding author:}

Prof. Claudia Schmidt-Dannert

140 Gortner Laboratory

University of Minnesota

1479 Gortner Avenue

St. Paul, MN 55108

E-mail: schmi232@umn.edu

Phone: +1 6126255782 


\begin{abstract}
Engineered living materials (ELMs) are a fast-growing area of research that combine approaches in synthetic biology and material science. Here, we engineer $B$. subtilis to become a living component of a silica material composed of self-assembling protein scaffolds for functionalization and cross-linking of cells. B. subtilis was engineered to display SpyTags on polar flagella for cell attachment and cross-linking of SpyCatcher modified secreted scaffolds. Through deletion of the autolysis LytC, endospore limited $B$. subtilis cells become a structural component of the material with spores for long-term storage of genetic programming. Known silica biomineralization peptides were screened and scaffolds designed for silica polymerization to fabricate biocomposite materials with enhanced mechanical properties. We show that the resulting ELM can be regenerated from a piece of silica material and that new functions can be readily incorporated by co-cultivation of engineered $B$. subtilis strains. We believe that this work will serve as a framework for the future design of resilient ELMs as functional, self-healing materials for use as responsive coatings and plasters.
\end{abstract}

\title{
Keywords
}

Engineered Living Materials, biocomposite, self-assembly, protein scaffolds, silica, Bacillus. 


\section{Introduction}

The design of cells capable of producing self-organizing, biocomposite materials has the potential to enable the fabrication of new types of functional living materials with the ability of self-fabrication and self-repair. Engineered living materials (ELMs) are therefore a new and fast-growing area of research that combines approaches in synthetic biology and material sciences ${ }^{1-4}$. The fabrication of most ELMs, however, has so far largely relied on physical methods for incorporating a living component in an external material ${ }^{4-7}$. Engineering of "truly" living materials where the living component actively facilitates material fabrication and organization is much more challenging. True ELMs have been created by engineering $E$. coli to produce an extracellular matrix from curli fibers ${ }^{8-25}$. Other types of extracellular matrices for ELM fabrication were created from secreted bacterial cellulose to embed microbial cells ${ }^{26,27}$ or from elastin-like polypeptides to attach Caulobacter cells via their protein S-layers ${ }^{28}$. Among these examples, the secretion of a polypeptide-based scaffolding system or matrix offers greater control over material assembly and functionalization due to the genetic programmability of polypeptide structures and functions.

Despite the advances in ELM design, the diversity of different ELM types capable of autonomous self-fabrication and regeneration is still small. In addition, ELM fabrication is currently limited to common chassis organisms that lack the resilience and long-term viability capabilities to withstand conditions outside of the laboratory. To broaden the ELM landscape, we therefore sought to engineer a resilient ELM biocomposite that uses the spore-forming bacteria $B$. subtilis as its living component for the secretion of protein scaffolds for cell cross-linking and silica biomineralization.

B. subtilis is an industrially used GRAS (Generally Recognized as Safe) bacteria known to have excellent protein secretion capabilities. It forms spores that remain viable for a long time ${ }^{29-31}$, allowing it to persist in our ELM under unfavorable conditions until revival. This was recently demonstrated by bioprinting of Bacillus spores in an agarose matrix ${ }^{6}$. For the secretion of a selfassembling protein matrix, we chose a highly robust scaffolding system that we have previously characterized and engineered for the attachment of different cargo proteins ${ }^{32-35}$. Scaffolds selfassemble from hexameric units of the bacterial microcompartment shell protein EutM. EutM scaffold building blocks are highly amenable to engineering and tolerate $\mathrm{N}$ - and C-terminal fusions, including a C-terminal SpyCatcher domain for the covalent linkage of SpyTag-modified proteins to scaffolds ${ }^{34,35}$. We therefore rationalized that $B$. subtilis could be engineered to secrete EutM-SpyCatcher (EutM-SpyC) building blocks and attach itself via the SpyTag-SpyCatcher system to the formed scaffolds to become both a structural and the living component of the formed material. Further, by engineering the scaffolds to display a biomineralization peptide (EutM-BM), 
scaffold-silicification could then yield a durable biocomposite material for future development into functional coatings or plasters (illustrated in Fig. 1).

In this work, we first optimized secretion by $B$. subtilis of our EutM scaffold building blocks which are not normally secreted and in addition, rapidly self-assemble both in vivo and in vitro into large structures ${ }^{32-38}$. We then show that $B$. subtilis can be engineered to display a SpyTag on cluster, polar flagellar for cell attachment and cross-linking of EutM scaffolds that constitute the protein matrix of our ELM. To allow our production strain to remain a structural component of the formed material even in its dormant state, we created an endospore forming strain. Screening of known silica biomineralization peptides then allowed us to design scaffolds for silica polymerization to fabricate biocomposite materials with enhanced mechanical properties. Finally, we confirmed that our ELM can be regenerated from a piece of silica material and that new functions can be incorporated into the material simply by co-cultivation of engineered $B$. subtilis strains. We believe that this work will serve as a framework for the future design of resilient ELMs as functional, selfhealing materials such as responsive coatings and plasters.

\section{Results and Discussion}

\section{Identification of growth conditions for ELM fabrication}

Several factors need to be considered for the fabrication of a living silica biocomposite material. Bacillus growth conditions must be identified that are permissible for silica biomineralization and preferably, do not create an overly discolored material. These conditions must also support scaffold building block expression and secretion during exponential to early stationary phase growth when cells are flagellated and have not yet initiated sporulation. Growth conditions for $B$. subtilis 168 (referred to as WT) were therefore evaluated using two different media; a Luria Bertani (LB) and a clear, buffered Spizizen minimal medium (SMM). Bacillus cells were transformed with a plasmid ( $\mathrm{pCT}$-empty) derived from our previously developed cumate ( $p$-isopropyl benzoate) inducible Bacillus expression vector (Table S1) ${ }^{39}$. Bacterial growth, media $\mathrm{pH}$, flagella presence, and sporulation were then analyzed for 4 days in $24 \mathrm{~h}$ intervals at 20 and $30^{\circ} \mathrm{C} \mathrm{(Fig.} \mathrm{S1,} \mathrm{S2} \mathrm{and}$ S3). Although $\mathrm{pH}$ is not typically considered in culture conditions, silica polymerization is optimal between $\mathrm{pH}$ 6.0-8.0 and at $\mathrm{pH}$ levels above and below this range polymerization is inhibited ${ }^{40}$. During growth, $B$. subtilis increasingly alkalifies the culture medium by switching from sugar to amino acid catabolism - releasing ammonia from tryptophan (SMM) or hydrolyzed amino acids from tryptone and yeast extract (LB) (along with the secretion of alkaline proteases) ${ }^{41}$. In buffered $\mathrm{SMM}$ at $20^{\circ} \mathrm{C}$, the $\mathrm{pH}$ was maintained around 7.0 until $48 \mathrm{~h}$ of growth, while under all other conditions tested and during longer growth, media pH quickly increased above 7.0 (Fig. S1). 
Peritrichous flagella were observed through $72 \mathrm{~h}$ of growth without significant sporulation (Fig. S2). Buffered SMM and growth for $48 \mathrm{~h}$ at $20^{\circ} \mathrm{C}$ therefore appeared to be optimal for our ELM production by engineered $B$. subtilis.

We then repeated these growth experiments with $B$. subtilis WT transformed with a plasmid (pCTEutMSpyC) for the secretion of the scaffolding protein EutM-SpyCatcher modified with the $\mathrm{N}$ terminal secretion peptide SacB from B. subtilis (Fig. 2a). As before, pH increased above 7.5 regardless of medium at $30^{\circ} \mathrm{C}$ after $24 \mathrm{~h}$ in LB and after $48 \mathrm{~h}$ in SMM. (Fig. S1). Again, only cultures grown in $\mathrm{SMM}$ at $20^{\circ} \mathrm{C}$ maintained a stable $\mathrm{pH}$ around 7.0 for $48 \mathrm{~h}$ and even until $96 \mathrm{~h}$ after induction of protein expression. Notably, growth was significantly reduced at $20^{\circ} \mathrm{C}$ in $\mathrm{SMM}$ and LB during $48 \mathrm{~h}$ after induction compared to the empty vector control cultures. This was most notable in SMM at $20^{\circ} \mathrm{C}$ (Fig. 2b), suggesting that EutM-SpyCatcher protein overexpression and secretion may burden cells, slowing cell growth. Like the empty vector cultures above, EutMSpyCatcher expressing cultures had peritrichous flagella through $72 \mathrm{~h}$ of growth, with the most flagella present until $48 \mathrm{~h}$ (Fig. S3a). However, spores were already observed after $48 \mathrm{~h}$ of growth at $20^{\circ} \mathrm{C}$ in LB media, indicating cells were stressed under this condition (Fig. S3b). Based on these results, we chose to perform all subsequent cultivations for ELM production in SMM at $20^{\circ} \mathrm{C}$ for $48 \mathrm{~h}$ as our standard condition.

\section{Establishing EutM scaffold building block secretion}

The majority of industrially produced recombinant proteins by $B$. subtilis are extracellular enzymes (e.g. hydrolases) and achieving high-level secretion typically requires the identification of efficient secretion signal peptides to replace their native secretion signals ${ }^{42-44}$. In contrast, our scaffolding protein EutM is cytoplasmic that also rapidly self-assembles into scaffolds when overexpressed in E. coli ${ }^{32-38}$. We therefore screened different signal peptides and investigated secretion of EutMSpyCatcher by Bacillus. In addition to SacB, we tested four other, commonly used secretion peptides (XynA, YngK, CelA and LipA) ${ }^{45-49}$ by fusing them to the $N$-terminus of our scaffold protein (Fig. S4a).

Each recombinant protein was expressed in $B$. subtilis WT in SMM, at $20^{\circ} \mathrm{C}$ for $48 \mathrm{~h}$ as the optimal condition confirmed above. Protein secretion levels were then compared between the different cultures by SDS-PAGE analysis. We analyzed total protein in the culture, and after centrifugation of cultures, protein in the resulting culture supernatant and pellet. However, a very low amount of secreted protein was observed in the supernatant compared to visible bands in the pellet, and we suspected that secreted and scaffolded EutM proteins were pelleting together with the cells. We therefore solubilized scaffold proteins from pelleted cells with $4 \mathrm{M}$ urea, which we have used 
previously to solubilize EutM scaffolds produced by E. coli ${ }^{33,35}$. At this concentration, urea does not lyse cells which we verified by comparing total protein released from completely lysed cells after urea treatment and protein solubilized from sediment (see Fig. S5). We compared by SDSPAGE three fractions as shown in Fig. 2c: (A) culture supernatants (concentrated by trichloroacetic acid (TCA)), (B) urea supernatant after scaffold solubilization from pellets, and (C) total protein released from remaining pellet after urea solubilization and cell lysis. Only the SacB secretion signal peptide yielded significant yields of secreted protein in the culture supernatant and the solubilized fraction (Fig. 2d). Only small amounts of secreted EutM protein (only after 25x concentration) could be detected in culture supernatants with the other four signal peptides (Fig. S4b). Compared to cultures expressing SacB-EutM-SpyCatcher, no scaffolds sedimented in the other cultures secreting low levels of EutM protein (Fig. S4b). From these results, we concluded that SacB is the best secretion signal peptide for EutM-SpyCatcher.

\section{Engineering B. subtilis cells as integral material component}

With scaffold building block secretion confirmed, we next set out to engineer our host strain to persist as a component within the matrix it fabricates by providing structure to the material and for material regeneration when favorable growth conditions are provided (Fig. 1). B. subtilis spores are extremely durable and can remain dormant indefinitely until proper environmental conditions return whereupon the spores will germinate ${ }^{29,31}$. During sporulation, endospores are formed in the mother cell which then undergoes lysis for spore release. To retain Bacillus cells as structural material components while also allowing spore formation for long-term viability of our material, we deleted the cell wall autolysin LytC ( $\mathrm{N}$-acetylmuramoyl-L-alanine amidase) in $B$. subtilis ${ }^{50}$ by in-frame deletion of $l y t C$ to generate a $B$. subtilis $\Delta l y t C$ strain (Fig. 3a and Fig. S6).

As our objective is to link $B$. subtilis cells to secreted EutM-SpyCatcher scaffold via SpyTag display on its flagella (Fig. 1), we also introduced a second in-frame deletion of flhG (Fig. 3a and Fig. S6) to change the flagellation pattern on cells from peritrichous to polar flagella clusters. Normally, B. subtilis produces flagella around the midcell and lacks flagella at the poles ${ }^{51}$. The protein FlhG (also called $\mathrm{YlxH}$, MinD2, FleN, and MotR) is essential to this flagellation pattern 52,53 . The deletion of $f / h G$ results in the aggregation of basal bodies at the polar ends of cells ${ }^{51}$. We reasoned that reducing flagellation density and directing flagella to the poles will enable more spaced-out cross-linking on scaffolds and less "clumping" of cells (which we later serendipitously discovered appears to be deleterious for $B$. subtilis, see below).

The resulting double deletion strain $B$. subtilis $\Delta / y t C \Delta f / h G$ was then transformed with expression vectors ( $\mathrm{pCT}$-empty, $\mathrm{pCT}$-EutMSpyC) for confirmation of expected cell phenotypes and scaffold 
building block secretion (Fig. 3b-d). Comparison of flagella pattern between wild-type B. subtilis and the double deletion strain confirmed the presence of clustered polar flagella and cell wall enclosed endospores in the deletion strain (Fig. 3b). Clustered polar flagella were most prevalent at $48 \mathrm{~h}$ or less after induction, and the most flagella both for the wild-type and the double deletion strain were observed in cultures grown at $20^{\circ} \mathrm{C}$ (Fig. S2a, S3a, S6d). Unlike the wild-type strain, no free spores were released by the autolysin deficient $\Delta / y t \mathrm{C}$ strain in SMM (Fig. S2b, S3b, S6c). Endospore formation by $B$. subtilis $\Delta / y t C \Delta$ flhG harboring pCT-empty and pCT-EutMSpyC was not observed until $72 \mathrm{~h}$ of growth under the optimal growth conditions $\left(\mathrm{SMM}, 20^{\circ} \mathrm{C}\right)$ identified above (Fig. 3b).

Following the confirmation of clustered flagella and no sporulation during growth in $\mathrm{SMM}$ at $20^{\circ} \mathrm{C}$ for $48 \mathrm{~h}$ after induction, we next evaluated growth and protein expression under these conditions (Fig. 3c, d). Interestingly, the growth rate of the double deletion strain was steadier and higher than the wild-type strain when transformed with either the empty control plasmid or pCTEutMSpyC (compare Fig. 2b and Fig. 3c). The pH of the culture was also maintained at around 7.0 for $48 \mathrm{~h}$. Expression and secretion of scaffold building blocks by the engineered strain was unchanged from the wild-type strain (Fig. 3d, compare to Fig. 2d) despite reaching a slightly higher optical density after $48 \mathrm{~h}$.

We noticed that the secreted EutM-SpyCatcher protein appears as a major higher $(24.4 \mathrm{kDa})$ and a minor lower $(21.1 \mathrm{kDa})$ molecular weight band on SDS-PAGE gels in all three analyzed fractions (culture supernatant, urea solubilized scaffolds and lysed cell pellet, Fig. 2d and $\mathbf{3 d}$ ). We previously observed that EutM proteins do not migrate according to size and/or as double bands $33,36,38$. Here, we wondered whether the differences in apparent molecular weights might be due to uncleaved SacB signal peptide. Secretion of native proteins with uncleaved Sec signal sequences into culture supernatants has been documented for bacteria ${ }^{54}$. LC-MS analysis showed that indeed the signal peptide was not cleaved from either EutM protein band (Fig. S7). This suggests that EutM likely folds rapidly upon emerging from the Sec translocase complex ${ }^{42,43}$, preventing access for the signal peptidase and pulling the folding protein into the extracellular space. EutM and its homologs are known to rapidly fold and self-assemble in vitro and in vivo to form higher order structures ${ }^{32-38}$. In contrast, the release and folding of the $B$. subtilis levansucrase, which is the source of the SacB secretion signal sequence, has been shown to be a slow process ${ }^{55}$.

To determine whether the remaining SacB signal peptide effects EutM-SpyCatcher assembly and to compare EutM-SpyCatcher protein assembly in SMM medium to previous observations in Tris 
buffer, we purified uncleaved and secreted protein from recombinant $B$. subtilis $\Delta / y t C \Delta f / h G$ cultures and His-EutM-SpyCatcher without signal sequence from recombinant $E$. coli cultures. After metal affinity chromatography and dialysis into SMM, scaffolds were observed by transmission electron microscopy (TEM). In comparison to the negative control of SMM which shows many nonhomogenously sized and poorly stained structures and a few heavily stained structures (Fig. S8a, e), scaffolds isolated from E. coli formed rolled-up tubes (Fig. S8b) which have been observed previously for EutM proteins expressed in E. coli ${ }^{33}$. Bacillus secreted protein assembled into fewer large, rolled up tubes and smaller, spherical structures (that stain as donutlike structures) as well as sheets and less-ordered coral-like scaffolds (Fig. S8c, d, f-h). These structures have also been previously observed for EutM homologs ${ }^{33,38}$. This greater variety of scaffold assemblies may be due to the SacB sequence, modifying surface charges and assembly. These results confirm that $B$. subtilis secreted EutM-SpyCatcher forms scaffolds and that the SMM growth medium does not inhibit self-assembly.

\section{Linking B. subtilis cells to secreted EutM-SpyCatcher scaffolds by SpyTag display}

We next set out to engineer the display of a SpyTag on flagella for cell attachment to secreted scaffolds. For characterization of flagella SpyTag display, a cysteine variant (T209C) of the flagellin Hag protein was first created for labeling of flagella filaments with a sulfhydryl-specific (maleimide) dye ${ }^{56}$. The modified flagellin gene was then expressed from its native promoter on a plasmid in wild-type $B$. subtilis and the $\Delta / y t C \Delta f l h G$ double deletion strain. The assembled flagella filaments are therefore composed of both engineered and native flagellin monomers. The recombinant $\mathrm{Hag}^{\mathrm{T} 209 \mathrm{C}}$ protein was then modified to include a SpyTag domain at nine different positions chosen inside the variable D2/D3-region of the flagellin protein (Fig. 4a, b) ${ }^{57}$. Four Hag insertions (bp positions 399, 426, 459, 537) were deleterious for both $B$. subtilis strains, meaning that either no transformants could be obtained or transformants contained missense mutations or deletions in the modified hag gene of the recovered plasmids (Fig. 4c). Fluorescence microscopy (Fig. 4d) of the remaining mutants revealed flagella located at the mid cell for $B$. subtilis WT and poles for $B$. subtilis $\Delta l y t C \Delta$ flhG which adopt different filament conformations (Fig. 4e, S9a). Wildtype flagella form a left-handed helix ${ }^{58}$, which was retained for insertions at positions 612 and 645 in $\mathrm{Hag}^{\mathrm{T} 209 \mathrm{C}}$. Curly, right-handed forms (with half the pitch and amplitude) were observed for flagella expressing SpyTags at positions 555 and 588. SpyTag insertion at position 588 also resulted in shortened flagella compared to unmodified flagella (Fig. 4e). One strain expressing Hag $^{\text {T209C }}$ with a SpyTag at position 492 had both normal and curly flagella (Fig. S9a). 
Functional display of the SpyTag on $B$. subtilis flagella by the different insertion mutants was then evaluated by adding purified red fluorescent tdTomato-SpyCatcher protein (Fig. 4d) to recombinant $B$. subtilis WT and $\Delta / y t C \Delta f / h G$ cultures that were then grown overnight for flagella expression. No attachment of tdTomato-SpyCatcher to flagella of strains with Hag ${ }^{\text {T209C }}$ SpyTag insertions at positions 492, 555, and 612 was observed by fluorescence microscopy (Fig. 4f, S9b). Some red fluorescent labeling was observed for SpyTag display at position 645 (Fig. S9b), but the best attachment of tdTomato-SpyCatcher was achieved on flagella that display a SpyTag at position $588\left(\mathrm{Hag}^{\mathrm{T} 209 \mathrm{C}}:: \mathrm{SpyT} \mathrm{Tag}^{588}\right)$, despite the shorter than wild-type flagella formed by the recombinant strains (Fig. 4f).

We then confirmed that Hag ${ }^{\top 209 C}:: S p y T a g^{588}$ modified flagella on $B$. subtilis $\Delta / y t C \Delta f / h G$ are capable of forming isopeptide bonds with EutM-SpyCatcher proteins secreted during cultivation. For this, two $B$. subtilis $\Delta l y t C \Delta f / h G$ strains - one expressing Hag ${ }^{\text {T209C_SpyTag }}{ }^{588}$ and the other EutM-SpyCatcher - were co-cultured under the identified conditions for scaffold building block secretion but extended to $96 \mathrm{~h}$ of induction (SMM, $20^{\circ} \mathrm{C}, 96 \mathrm{~h}$ induction). Flagella were then isolated from cells and analyzed by SDS-PAGE, confirming the presence of a higher-molecular weight protein complex (55 kDa) that was not present in the control (Fig. $\mathbf{4 g}$ ). Based on these results, we therefore chose $\mathrm{Hag}^{\mathrm{T209C}:: S p y T a{ }^{588}}$ expression for cross-linking B. subtilis $\Delta / y t C$ $\Delta$ flhG cells to EutM-SpyCatcher scaffolds.

\section{Engineering of silica biomineralization}

Our next objective was to modify EutM scaffolds such that they would biomineralize silica for the fabrication of a resilient living biocomposite material. We selected four known silica biomineralization peptides (R5, CotB1p, SB7, and Synthetic ${ }^{59-63}$ for C-terminal fusion to His-EutM (Fig. S10a). We then characterized silica precipitation and confirmed scaffold formation with the fusion proteins overexpressed and purified from E. coli. We intentionally left the His-Tag on our scaffold building blocks as histidine residues are enriched in proteins involved in natural silica biomineralization processes such as e.g. in the histidine-rich glassin protein present in the siliceous skeletons of sponges ${ }^{64}$.

Silica precipitation by the modified EutM scaffolds was then quantified using a spectroscopic molybdate blue assay ${ }^{65}$ (Fig. S10a). The CotB1p (hereafter referred to as CotB) peptide fusion was most efficient at precipitating silica at $7.44 \pm 0.36 \mathrm{mmol} / \mathrm{g}$ protein and was also the most cationic peptide, rich in arginine residues. As expected, His-EutM scaffolds also precipitated silica at $1.98 \pm 0.01 \mathrm{mmol} / \mathrm{g}$ protein. The structures (scaffolds) formed by the fusion proteins and HisEutM were then visualized by SEM (Fig. 5a and Fig. S10b, left column). His-EutM formed tubular 
nanostructures that appeared to be rolled-up tubes fused together at multiple locations. In contrast, His-EutM-R5 formed globular structures fused into chains, while His-EutM-CotB formed similar scaffolds but with a greater surface roughness. Fusion of the SB7 and Synthetic peptide to EutM resulted in the formation of dense scaffolds comprised of fused together globular and poorly-formed tubular structures (Fig. S10b).

Silica precipitation on scaffolds was investigated by incubating $1 \mathrm{mg} / \mathrm{mL}$ scaffolds for $2 \mathrm{~h}$ at room temperature with $100 \mathrm{mM}$ silica. SEM imaging of scaffolds (Fig. 5a, center and right) showed an increase in scaffold surface roughness, surface porosity, and the presence of silica nanoparticles fused to the scaffolds which are characteristics of silicification (Fig. 5a and Fig. S10b, center and right columns). Silica nanoparticle formation was most prominent on His-EutM and His-EutM-SB7 scaffolds, suggesting that biosilicification may have been nucleated in the solution phase by these proteins. In contrast, a significant increase in surface roughness seen for His-EuM-R5 and HisEutM-CotB scaffolds suggests surface catalyzed biomineralization by these structures.

Based on these results, we chose the CotB biomineralization peptide for fusion to scaffold building blocks for secretion by $B$. subtilis. An expression construct was made where the SpyCatcher domain in $\mathrm{pCT}$-EutMSpyC was replaced with CotB to yield plasmid $\mathrm{pCT}$-EutMCotB, replicating the E. coli expressed His-EutM-CotB with the additional SacB signal sequence (Fig. 5b). EutMCotB expression and secretion by $B$. subtilis $\Delta / y t C \Delta f / h G$ transformed with pCT-EutMCotB (SMM, $20^{\circ} \mathrm{C}, 48 \mathrm{~h}$ induction) was confirmed by SDS-PAGE (Fig. 5c, lane 3). As in the case of EutMSpyCatcher, the SacB signal sequence appears to be uncleaved based on the size of the observed protein band (16.8 kDa vs $13.8 \mathrm{kDa}$ for the uncleaved and cleaved proteins, respectively). Secretion of EutM-CotB however was much lower than EutM-SpyCatcher, probably due to the added positive charged by the CotB peptide fusion ${ }^{66}$. Yet, despite the low amount of secreted EutM-CotB, cultures of $B$. subtilis strains transformed with pCT-EutMCotB significantly increased silica gel formation at low silica concentrations compared to empty vector control cultures (Fig. S11). After $48 \mathrm{~h}$ induction of protein expression (SMM, $20^{\circ} \mathrm{C}$ ), samples of cultures were incubated with different concentrations of silica $(50-500 \mathrm{mM})$ and silica gel formation followed for $24 \mathrm{~h}$ at $20^{\circ} \mathrm{C}$. With $100 \mathrm{mM}$ silica added, the EutMCotB expressing cultures solidified into a gel, while the control cultures only formed a liquid soft gel (Fig. S11).

We then proceeded with combining the expression and secretion of our EutM-SpyCatcher building block for cell cross-linking with the EutM-CotB scaffold building block for enhanced biomineralization, yielding the expression vectors as shown in Fig. 5b. For SpyTag-display on flagella of $B$. subtilis $\Delta l y t C \Delta f / h G$, a plasmid for co-expression of EutM scaffold building blocks 
with the modified flagellin subunit $\mathrm{Hag}^{\mathrm{T} 209 \mathrm{C}:: S p y T a{ }^{588}}$ was also constructed. Each expression module contains its own promoter as shown. EutM building block expression and secretion by $B$. subtilis $\triangle l y t C \Delta f l h G$ transformed with the different plasmids was then confirmed and compared, showing again lower secretion levels for EutM-CotB compared to co-expressed EutM-SpyCatcher in SMM at $20^{\circ} \mathrm{C}$ after $48 \mathrm{~h}$ of induction (Fig. 5c). We also attempted to visualize by fluorescence microscopy the flagella phenotypes of $B$. subtilis $\Delta / y t C \Delta f l h G$ cultures transformed with the EutM co-expression constructs with and without $\mathrm{Hag}^{\mathrm{T} 209 \mathrm{C}}:$ :SpyTag ${ }^{588}$. We observed large structures labeled with cysteine reactive dye that interfere with cell labeling (Fig. S12). In contrast, cells that expressed only the modified $\mathrm{Hag}^{\mathrm{T} 209 \mathrm{C}}$ or $\mathrm{Hag}^{\mathrm{T} 209 \mathrm{C}}:$ :SpyTag ${ }^{588}$ contained clearly labeled flagella under the same growth conditions (Fig. S12). We therefore presume that the dye labeled structures represent secreted EutM scaffold where one or both cysteine residues of the EutM proteins are labeled.

\section{Biocomposite ELM fabrication and characterization}

For the final fabrication of our biocomposite ELM we performed biomineralization experiments with induced and uninduced (control) cultures of $B$. subtilis $\Delta / y t C \Delta f l h G$ transformed with an empty control plasmid or one of the EutM-SpyCatcher and EutM-CotB co-expression plasmids with or without $\mathrm{Hag}^{\text {T209C.::SpyTag }}{ }^{588}$. After $48 \mathrm{~h}$ of cultivation, $5 \mathrm{~mL}$ culture samples were transferred and further incubated with and without $100 \mathrm{mM}$ silica in 6-well plates at $20^{\circ} \mathrm{C}, 100 \mathrm{rpm}$ for $1 \mathrm{~h}$ (Fig. $6 \mathrm{a}$, see Fig. S13 for biological replicates). While all cultures show some silica condensation into clusters at charged surfaces present in the cultures, cultures that expressed and secreted EutM scaffolds (induced cultures) formed aggregated silica materials. Larger silica material aggregates were formed in cultures that also displayed SpyTagged flagella for material cross linking.

To test whether the silica materials made by the engineered Bacillus strains above have different mechanical properties due to scaffold secretion and cross-linking, we optimized conditions for the formation silica blocks suitable for the rheological analysis (Fig. 6b). Rheological measurements have also been performed for other types of living materials, such as for example a cellulosebased material of a bacterial and yeast co-cultures with programmable weakening of material microstructure ${ }^{27}$. For measurements of our materials, induced culture samples from Fig. 6a were incubated in a syringe with $200 \mathrm{mM}$ silica at $20^{\circ} \mathrm{C}$ or $25^{\circ} \mathrm{C}$ for up to $5 \mathrm{~h}$ until solid gel blocks were obtained $\left(25^{\circ} \mathrm{C}, 5 \mathrm{~h}\right)$ for measurements with a rheometer. Small, but significant increases in storage moduli (G') were measured for cultures co-expressing EutM scaffold building blocks (1.23-fold for pCT-EutMCotB-EutMSpyC and 1.37-fold for pCT-EutMCotB-EutMSpyC-

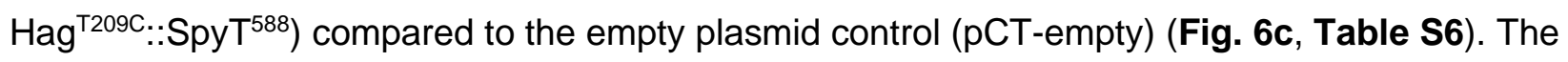


loss modulus (G") remained the same for all samples and was lower than G' as would be expected for materials with lower viscous dissipation. The increases in G' shows that the silica gel blocks from scaffold secreting cultures have a higher degree of intermolecular bonding giving them greater mechanical rigidity. The engineered Bacillus strain with the SpyTag displaying flagella yielded the most robust material due to cross-linking of cells and scaffolds.

Although we have shown that our final ELM Bacillus strain (B. subtilis $\Delta / y t C \Delta$ flhG pCT-EutMCotBEutMSpyC-Hag ${ }^{T 209 C}::$ Spy ${ }^{588}$ ) improved material strength, we wanted to determine where the secreted scaffolds are located in the material. Uninduced and induced cultures used for silica material formation in Fig. 6 were incubated with SpyTag-eGFP or eGFP (control) to label SpyCatcher domains on EutM scaffolds not linked to SpyTag displaying flagella of cells (Fig.7a). No labeling was observed with uninduced or eGFP control samples. In contrast, regions outside and in between cells were labeled in the induced samples, suggesting that EutM proteins are secreted and assemble into scaffolds between the cells. The relatively high binding of SpyTageGFP indicates that there are many available binding sites for future incorporation of additional, secreted SpyTagged cargo proteins for the fabrication of functional ELMs.

The same cultures examined by GFP labeling were then transformed into a silica blocks as in Fig. $\mathbf{6 b}$ for TEM visualization of cells in the biocomposite material. After curing, solid gel plugs were cut into $2 \mathrm{~mm}^{3}$ pieces and processed for thin sectioning. Cell were observed in clusters for both the induced and uninduced cultures in relatively low density which corresponds to a final $\mathrm{OD}_{600}$ of $\sim 3.5$ (induced) or $\sim 6$ (uninduced) of the cultures prior to silica material formation (Fig. 7b). Unexpectedly, no scaffolds could be visualized outside or in between cells in the material from the induced cultures. Instead, only thin sections from the induced cultures contained large clear zones around cells. These zones correspond to the SpyTag-eGFP labeled areas (Fig. 7a) and indicates that a material is physically taking up the space that does not stain after silica biomineralization. The same observations were made when the cell density in silica blocks was increased to an $\mathrm{OD}_{600}$ of 10 and 20 by gentle centrifugation and resuspension of cells prior to biomineralization (Fig. S14 $\mathbf{b}$ and $\mathbf{c}$ ). We therefore theorize that silica deposition on EutM scaffolds surrounding the cells prevents staining. Although no scaffolds were visualized in thin sections, silica gels made in the same manner above show protein bands of the expected sizes for both EutM-SpyCatcher and biomineralizing scaffolds on SDS-PAGE analysis (Fig. S14a) supporting our theory that silica biomineralization is interfering with staining scaffolds during thin sectioning.

\section{Regeneration and functional augmentation of biocomposite ELM}


Ultimately, a living composite material for application as e.g. functional coating or plaster should be self-regenerating and integrate desired functions. We therefore first tested whether our ELM could be regenerated from a small plug $\left(\sim 5 \mathrm{~mm}^{3}\right)$ taken from silica blocks generated from cultures of our final ELM strain. Blocks fabricated with $200 \mathrm{mM}$ silica were cured for 1 day at $25^{\circ} \mathrm{C}$ and then small pieces were used to inoculate fresh cultures for expression and secretion of scaffold building blocks (Fig. 8a). After $48 \mathrm{~h}$ of growth with and without induction (control), secretion of EutM proteins at similar levels than the parent culture could be confirmed in the induced cultures (Fig. 8b). Similarly, we could reproduce the formation of cross-linked silica material in 6-well plates with the induced cultures (Fig. 8c). These results show that the recombinant Bacillus cells remain viable in the silica material and retain their engineered functions. We repeated the same experiments with silica blocks that were cured for 2 weeks at $25^{\circ} \mathrm{C}$. Although cultures could be regrown in selective media, meaning that the plasmid is still present, no scaffold building block secretion could be detected. Due to plasmid-based expression of our proteins, we theorized this issue might be caused by mutations in their coding sequences. Sequencing of plasmids isolated from individual colonies derived from the revived culture indeed confirmed that all of them contained multiple mutation in the EutM gene regions. The rapid mutability of high copy number plasmids in Bacillus is a well-known issue ${ }^{67,68}$ and can be solved in the future by integrating the expression cassettes currently located on a plasmid into the genome of our engineered Bacillus strain. Nevertheless, the ability to regrow the engineered strain with its plasmid after 2 weeks in a silica gel speaks to the resilience of $B$. subtilis as ELM chassis organism.

As a proof-of-concept, we sought to demonstrate the incorporation of another property into our material. Rather than adding another genetic burden to our engineered Bacillus strain, we opted to co-cultivate our engineered strain with a second engineered Bacillus strain that would provide the new property. Such a co-cultivation strategy but with two different microorganisms has recently been successfully explored for the fabrication of a living material that incorporates cellulose ${ }^{27}$. For demonstration purposes, we chose the formation of colored material by expressing a purple chromoprotein ${ }^{69}$. An additional plasmid pCT-Purple-Hag ${ }^{\mathrm{T} 209 \mathrm{C}}: . \mathrm{Spy}^{588}$ was constructed for transformation into $B$. subtilis $\Delta / y t C \Delta f / h G$ and co-cultivation with our final ELM strain. Co-cultures (mixed at a 1:1 ratio in the induction step) were grown as before (Fig. 6) and biomineralization and silica material formation compared to co-cultures with an empty plasmid control (Fig. 8d). Only co-cultures that also expressed the EutM scaffolds formed a purple a crosslinked silica material due to the attachment of the purple protein expressing Bacillus cells (which also display SpyTags on their flagella) to the scaffolds. Likewise, a purple-colored silica block could be fabricated under the same conditions used above with monocultures of our engineered 
Bacillus strain (Fig. 8e). These results demonstrate an easy and modular approach for the fabrication of living materials with tailored functions and properties ranging e.g. from color and antimicrobial properties ${ }^{70}$ to more complex functions such as sensing and responding.

\section{CONCLUSIONS}

We successfully engineered $B$. subtilis to produce and secrete proteins that self-assemble into scaffolds capable of biomineralizing silica, forming a material with tailorable properties and functions via its ability to crosslink cells and covalent attachment of cargo proteins. Our ELM system does not rely upon special processing after cultivation and instead just requires the addition of silica. Microscopy and rheological data show this ELM system function effectively to secrete biomineralizing and cross-linking scaffold that produce a material with stronger mechanical properties than the non-scaffold secreting controls. Although silica gel formation requires a concentration of $100 \mathrm{mM}$ silica, which is currently much higher than used by natural systems which can biomineralize silica through concentration mechanisms from less than $100 \mu \mathrm{M}$ available silica in their environments ${ }^{71}$, the use of hydrolyzed TEOS as a source of silica is inexpensive. Additional research to enhance biomineralization by engineering and increasing the density of biomineralization peptides on scaffolds could reduce silica concentrations, and the incorporation of different types of biomineralization peptides ${ }^{59-63}$ would give access to a range of other ELM biocomposite materials. We have shown that our ELM can be regenerated from a piece of silica material after $24 \mathrm{~h}$ of curing. But extended material storage and regeneration attempts resulted in the accumulation of mutations in plasmids recovered from regrown cells. We utilized plasmids for easy optimization of protein expression, but these expression constructs once designed in their final configurations will need to be integrated into the genome to ensure stable, genetic programming of ELMs that can regenerated over many cycles. Finally, this work offers engineering strategies for expanding the repertoire of current ELM chassis organisms towards other spore forming and/or ultra-resilient bacteria that secrete and embed themselves into a genetically programmable protein matrix for the fabrication of a range of functional materials.

\section{METHODS}

Methods and materials are provided in the Supplementary Information. 


\section{REFERENCES}

1 Chen, A. Y., Zhong, C. \& Lu, T. K. Engineering living functional materials. ACS Synth Biol 4, 8-11, doi:10.1021/sb500113b (2015).

2 Tang, T.-C. et al. Materials design by synthetic biology. Nature Reviews Materials, doi:10.1038/s41578-020-00265-w (2020).

3 Srubar, W. V., 3rd. Engineered living materials: Taxonomies and emerging trends. Trends Biotechnol, doi:10.1016/j.tibtech.2020.10.009 (2020).

4 Rivera-Tarazona, L. K., Campbell, Z. T. \& Ware, T. H. Stimuli-responsive engineered living materials. Soft Matter, doi:10.1039/d0sm01905d (2021).

5 Liu, X. et al. 3D printing of living responsive materials and devices. Adv Mater 30, doi:10.1002/adma.201704821 (2018).

6 Gonzalez, L. M., Mukhitov, N. \& Voigt, C. A. Resilient living materials built by printing bacterial spores. Nat Chem Biol 16, 126-133, doi:10.1038/s41589-019-0412-5 (2020).

$7 \mathrm{Yu}, \mathrm{K}$. et al. Photosynthesis-assisted remodeling of three-dimensional printed structures. Proc Natl Acad Sci USA 118, doi:10.1073/pnas.2016524118 (2021).

8 Lovley, D. R. \& Yao, J. Intrinsically conductive microbial nanowires for 'Green' electronics with novel functions. Trends Biotechnol, doi:10.1016/j.tibtech.2020.12.005 (2021).

9 Saldanha, D. J., Abdali, Z., Modafferi, D., Janfeshan, B. \& Dorval Courchesne, N. M. Fabrication of fluorescent $\mathrm{pH}$-responsive protein-textile composites. Sci Rep 10, 13052, doi:10.1038/s41598020-70079-x (2020).

10 Levkovich, S. A., Gazit, E. \& Laor Bar-Yosef, D. Two decades of studying functional amyloids in microorganisms. Trends Microbiol, doi:10.1016/j.tim.2020.09.005 (2020).

11 Balistreri, A., Kahana, E., Janakiraman, S. \& Chapman, M. R. Tuning functional amyloid formation through disulfide engineering. Front Microbiol 11, 944, doi:10.3389/fmicb.2020.00944 (2020).

12 Abdali, Z. et al. Curli-mediated self-assembly of a fibrous protein scaffold for hydroxyapatite mineralization. ACS Synth Biol 9, 3334-3343, doi:10.1021/acssynbio.0c00415 (2020).

13 Mao, X. et al. Directing curli polymerization with DNA origami nucleators. Nat Commun 10, 1395, doi:10.1038/s41467-019-09369-6 (2019).

14 Kalyoncu, E., Ahan, R. E., Ozcelik, C. E. \& Seker, U. O. S. Genetic logic gates enable patterning of amyloid nanofibers. Adv Mater 31, e1902888, doi:10.1002/adma.201902888 (2019).

15 Duraj-Thatte, A. M. et al. Genetically programmable self-regenerating bacterial hydrogels. Adv Mater 31, e1901826, doi:10.1002/adma.201901826 (2019).

16 Cohen, N., Zhou, H., Hay, A. G. \& Radian, A. Curli production enhances clay-E. coli aggregation and sedimentation. Colloids Surf B Biointerfaces 182, 110361, doi:10.1016/j.colsurfb.2019.110361 (2019).

17 Wang, X. et al. Programming cells for dynamic assembly of inorganic nano-objects with spatiotemporal control. Adv Mater 30, e1705968, doi:10.1002/adma.201705968 (2018).

18 Dorval Courchesne, N. M. et al. Biomimetic engineering of conductive curli protein films. Nanotechnology 29, 454002, doi:10.1088/1361-6528/aadd3a (2018).

19 Axpe, E. et al. Fabrication of amyloid curli fibers-alginate nanocomposite hydrogels with enhanced stiffness. ACS Biomater Sci Eng 4, 2100-2105, doi:10.1021/acsbiomaterials.8b00364 (2018).

20 Tay, P. K. R., Nguyen, P. Q. \& Joshi, N. S. A Synthetic circuit for mercury bioremediation using self-assembling functional amyloids. ACS Synth Biol 6, 1841-1850, doi:10.1021/acssynbio.7b00137 (2017). 
Seker, U. O., Chen, A. Y., Citorik, R. J. \& Lu, T. K. Synthetic biogenesis of bacterial amyloid nanomaterials with tunable inorganic-organic interfaces and electrical conductivity. ACS Synth Biol 6, 266-275, doi:10.1021/acssynbio.6b00166 (2017).

22 Dorval Courchesne, N. M., Duraj-Thatte, A., Tay, P. K. R., Nguyen, P. Q. \& Joshi, N. S. Scalable production of genetically engineered nanofibrous macroscopic materials via filtration. ACS Biomater Sci Eng 3, 733-741, doi:10.1021/acsbiomaterials.6b00437 (2017).

23 Botyanszki, Z., Tay, P. K., Nguyen, P. Q., Nussbaumer, M. G. \& Joshi, N. S. Engineered catalytic biofilms: Site-specific enzyme immobilization onto E. coli curli nanofibers. Biotechnol Bioeng 112, 2016-2024, doi:10.1002/bit.25638 (2015).

24 Nguyen, P. Q., Botyanszki, Z., Tay, P. K. \& Joshi, N. S. Programmable biofilm-based materials from engineered curli nanofibres. Nat Commun 5, 4945, doi:10.1038/ncomms5945 (2014). Drogue, B., Thomas, P., Balvay, L., Prigent-Combaret, C. \& Dorel, C. Engineering adherent bacteria by creating a single synthetic curli operon. J Vis Exp, e4176, doi:10.3791/4176 (2012). Caro-Astorga, J., Walker, K. T. \& Ellis, T. Bacterial cellulose spheroids as building blocks for 2D and 3D engineered living materials. bioRxiv, 2020.2005.2011.088138, doi:10.1101/2020.05.11.088138 (2020).

27 Gilbert, C. et al. Living materials with programmable functionalities grown from engineered microbial co-cultures. Nat Mater 20, 691-700, doi:10.1038/s41563-020-00857-5 (2021). Orozco-Hidalgo, M. T. et al. Engineering high-yield biopolymer secretion creates an extracellular protein matrix for living materials. mSystems 6, e00903-00920, doi:10.1128/mSystems.00903-20 (2021).

29 Cano, R. J. \& Borucki, M. K. Revival and identification of bacterial spores in 25- to 40-millionyear-old Dominican amber. Science 268, 1060-1064, doi:10.1126/science.7538699 (1995). Schallmey, M., Singh, A. \& Ward, O. P. Developments in the use of Bacillus species for industrial production. Can J Microbiol 50, 1-17, doi:10.1139/w03-076 (2004).

31 Ulrich, N. et al. Experimental studies addressing the longevity of Bacillus subtilis spores - The first data from a 500-year experiment. PLoS One 13, e0208425, doi:10.1371/journal.pone.0208425 (2018).

32 Choudhary, S., Quin, M. B., Sanders, M. A., Johnson, E. T. \& Schmidt-Dannert, C. Engineered protein nano-compartments for targeted enzyme localization. PLoS One 7, e33342, doi:10.1371/journal.pone.0033342 (2012).

33 Schmidt-Dannert, S., Zhang, G., Johnston, T., Quin, M. B. \& Schmidt-Dannert, C. Building a toolbox of protein scaffolds for future immobilization of biocatalysts. Appl Microbiol Biotechnol 102, 8373-8388, doi:10.1007/s00253-018-9252-6 (2018).

34 Zhang, G. Q., Quin, M. B. \& Schmidt-Dannert, C. Self-assembling protein scaffold system for easy in vitro coimmobilization of biocatalytic cascade enzymes. Acs Catal 8, 5611-5620, doi:10.1021/acscatal.8b00986 (2018).

35 Zhang, G., Schmidt-Dannert, S., Quin, M. B. \& Schmidt-Dannert, C. Protein-based scaffolds for enzyme immobilization. Methods Enzymol 617, 323-362, doi:10.1016/bs.mie.2018.12.016 (2019).

36 Held, M. et al. Engineering formation of multiple recombinant Eut protein nanocompartments in E. coli. Sci Rep 6, 24359, doi:10.1038/srep24359 (2016).

37 Quin, M. B., Perdue, S. A., Hsu, S. Y. \& Schmidt-Dannert, C. Encapsulation of multiple cargo proteins within recombinant Eut nanocompartments. Appl Microbiol Biotechnol 100, 9187-9200, doi:10.1007/s00253-016-7737-8 (2016).

38 Zhang, G., Johnston, T., Quin, M. B. \& Schmidt-Dannert, C. Developing a protein scaffolding system for rapid enzyme immobilization and optimization of enzyme functions for biocatalysis. ACS Synth Biol 8, 1867-1876, doi:10.1021/acssynbio.9b00187 (2019). 

system for Bacillus. Appl Microbiol Biotechnol 103, 303-313, doi:10.1007/s00253-018-9485-4 (2019).

40 Belton, D. J., Deschaume, O. \& Perry, C. C. An overview of the fundamentals of the chemistry of silica with relevance to biosilicification and technological advances. FEBS J 279, 1710-1720, doi:10.1111/j.1742-4658.2012.08531.x (2012).

41 Commichau, F. M., Gunka, K., Landmann, J. J. \& Stulke, J. Glutamate metabolism in Bacillus subtilis: gene expression and enzyme activities evolved to avoid futile cycles and to allow rapid responses to perturbations of the system. J Bacteriol 190, 3557-3564, doi:10.1128/JB.00099-08 (2008).

42 Freudl, R. Signal peptides for recombinant protein secretion in bacterial expression systems. Microb Cell Fact 17, 52, doi:10.1186/s12934-018-0901-3 (2018).

43 Song, Y., Nikoloff, J. M. \& Zhang, D. Improving protein production on the level of regulation of both expression and secretion pathways in Bacillus subtilis. J Microbiol Biotechnol 25, 963-977, doi:10.4014/jmb.1501.01028 (2015).

44 Su, Y., Liu, C., Fang, H. \& Zhang, D. Bacillus subtilis: a universal cell factory for industry, agriculture, biomaterials and medicine. Microb Cell Fact 19, 173, doi:10.1186/s12934-02001436-8 (2020).

45 Edelman, A., Joliff, G., Klier, A. \& Rapoport, G. A system for the inducible secretion of proteins from Bacillus-Subtilis during logarithmic growth. Fems Microbiol Lett 52, 117-120, doi:10.1111/j.1574-6968.1988.tb02581.x (1988).

46 Gilbert, C., Howarth, M., Harwood, C. R. \& Ellis, T. Extracellular self-assembly of functional and tunable protein conjugates from Bacillus subtilis. ACS Synth Biol 6, 957-967, doi:10.1021/acssynbio.6b00292 (2017).

47 Li, X. L. \& Ljungdahl, L. G. Expression of Aureobasidium pullulans xynA in, and secretion of the xylanase from, Saccharomyces cerevisiae. Appl Environ Microbiol 62, 209-213, doi:10.1128/AEM.62.1.209-213.1996 (1996).

48 Malten, M. et al. A Bacillus megaterium plasmid system for the production, export, and one-step purification of affinity-tagged heterologous levansucrase from growth medium. Appl Environ Microbiol 72, 1677-1679, doi:10.1128/AEM.72.2.1677-1679.2006 (2006).

49 Stammen, S. et al. High-yield intra- and extracellular protein production using Bacillus megaterium. Appl Environ Microbiol 76, 4037-4046, doi:10.1128/AEM.00431-10 (2010).

50 Smith, T. J. \& Foster, S. J. Characterization of the involvement of two compensatory autolysins in mother cell lysis during sporulation of Bacillus subtilis 168. J Bacteriol 177, 3855-3862, doi:10.1128/jb.177.13.3855-3862.1995 (1995).

51 Schuhmacher, J. S. et al. MinD-like ATPase FlhG effects location and number of bacterial flagella during C-ring assembly. Proc Natl Acad Sci USA 112, 3092-3097, doi:10.1073/pnas.1419388112 (2015).

52 Guttenplan, S. B., Shaw, S. \& Kearns, D. B. The cell biology of peritrichous flagella in Bacillus subtilis. Mol Microbiol 87, 211-229, doi:10.1111/mmi.12103 (2013).

53 Mukherjee, S. \& Kearns, D. B. The structure and regulation of flagella in Bacillus subtilis. Annu Rev Genet 48, 319-340, doi:10.1146/annurev-genet-120213-092406 (2014).

54 de Souza, G. A., Leversen, N. A., Malen, H. \& Wiker, H. G. Bacterial proteins with cleaved or uncleaved signal peptides of the general secretory pathway. J Proteomics 75, 502-510, doi:10.1016/j.jprot.2011.08.016 (2011).

55 Petit-Glatron, M. F., Benyahia, F. \& Chambert, R. Secretion of Bacillus subtilis levansucrase: a possible two-step mechanism. Eur J Biochem 163, 379-387, doi:10.1111/j.14321033.1987.tb10810.x (1987). 
56 Turner, L., Zhang, R., Darnton, N. C. \& Berg, H. C. Visualization of flagella during bacterial swarming. J Bacteriol 192, 3259-3267, doi:10.1128/JB.00083-10 (2010).

57 Wang, F. et al. A structural model of flagellar filament switching across multiple bacterial species. Nat Commun 8, 960, doi:10.1038/s41467-017-01075-5 (2017).

58 Beatson, S. A., Minamino, T. \& Pallen, M. J. Variation in bacterial flagellins: from sequence to structure. Trends Microbiol 14, 151-155, doi:10.1016/j.tim.2006.02.008 (2006).

59 Makin, O. S., Atkins, E., Sikorski, P., Johansson, J. \& Serpell, L. C. Molecular basis for amyloid fibril formation and stability. Proc Natl Acad Sci USA 102, 315-320, doi:10.1073/pnas.0406847102 (2005).

60 Abdelhamid, M. A. et al. Affinity purification of recombinant proteins using a novel silica-binding peptide as a fusion tag. Appl Microbiol Biotechnol 98, 5677-5684, doi:10.1007/s00253-014-5754z (2014).

61 Al-Garawi, Z. S., Thorpe, J. R. \& Serpell, L. C. Silica nanowires templated by amyloid-like fibrils. Angewandte Chemie-International Edition 54, 13327-13331, doi:10.1002/anie.201508415 (2015).

62 Senior, L. et al. Structure and function of the silicifying peptide R5. J Mater Chem B 3, 26072614, doi:10.1039/c4tb01679c (2015).

63 Abdelhamid, M. A. et al. Application of volcanic ash particles for protein affinity purification with a minimized silica-binding tag. J Biosci Bioeng 122, 633-638, doi:10.1016/j.jbiosc.2016.04.011 (2016).

64 Shimizu, K. et al. Glassin, a histidine-rich protein from the siliceous skeletal system of the marine sponge Euplectella, directs silica polycondensation. Proc Natl Acad Sci USA 112, 11449-11454, doi:10.1073/pnas.1506968112 (2015).

65 Coradin, T., Eglin, D. \& Livage, J. The silicomolybdic acid spectrophotometric method and its application to silicate/biopolymer interaction studies. Spectroscopy-an International Journal 18, 567-576, doi:10.1155/2004/356207 (2004).

66 Stephenson, K., Jensen, C. L., Jorgensen, S. T., Lakey, J. H. \& Harwood, C. R. The influence of secretory-protein charge on late stages of secretion from the Gram-positive bacterium Bacillus subtilis. Biochem J 350 Pt 1, 31-39, doi:10.1042/0264-6021:3500031 (2000).

67 Summers, D. K. \& Sherratt, D. J. Multimerization of high copy number plasmids causes instability: ColE1 encodes a determinant essential for plasmid monomerization and stability. Cell 36, 1097-1103, doi:10.1016/0092-8674(84)90060-6 (1984).

68 Alonso, J. C., Viret, J. F. \& Tailor, R. H. Plasmid maintenance in Bacillus subtilis recombinationdeficient mutants. Mol Gen Genet 208, 349-352, doi:10.1007/BF00330464 (1987).

69 Liljeruhm, J. et al. Engineering a palette of eukaryotic chromoproteins for bacterial synthetic biology. J Biol Eng 12, 8, doi:10.1186/s13036-018-0100-0 (2018).

70 Vera-Gonzalez, N. \& Shukla, A. Advances in biomaterials for the prevention and disruption of Candida biofilms. Front Microbiol 11, 538602, doi:10.3389/fmicb.2020.538602 (2020).

71 Treguer, P. et al. The silica balance in the world ocean: a reestimate. Science 268, 375-379, doi:10.1126/science.268.5209.375 (1995).

72 Altegoer, F. et al. Flis/flagellin/FliW heterotrimer couples type III secretion and flagellin homeostasis. Sci Rep 8, 11552, doi:10.1038/s41598-018-29884-8 (2018). 


\section{ACKNOWLEDGEMENTS}

This research was sponsored by the Defense Advanced Research Projects Agency (DARPA contract HR0011-17-2-0038) and supported by seed grants from the Biotechnology Institute at the University of Minnesota. Parts of this work were carried out in the University of Minnesota's Characterization Facility, which receives partial support from the NSF through the MRSEC (Award Number DMR-2011401) and NNCI (Award Number ECCS-2025124) programs. We also acknowledge resources and staff at the University of Minnesota core facilities for contribution to this work: Imaging Center (Dr. Gail Celico, sample thin sectioning) and Center for Mass Spectrometry (LC-MS sample analysis, Dr. LeeAnn Higgins and Todd Markowski). 


\section{FIGURES}

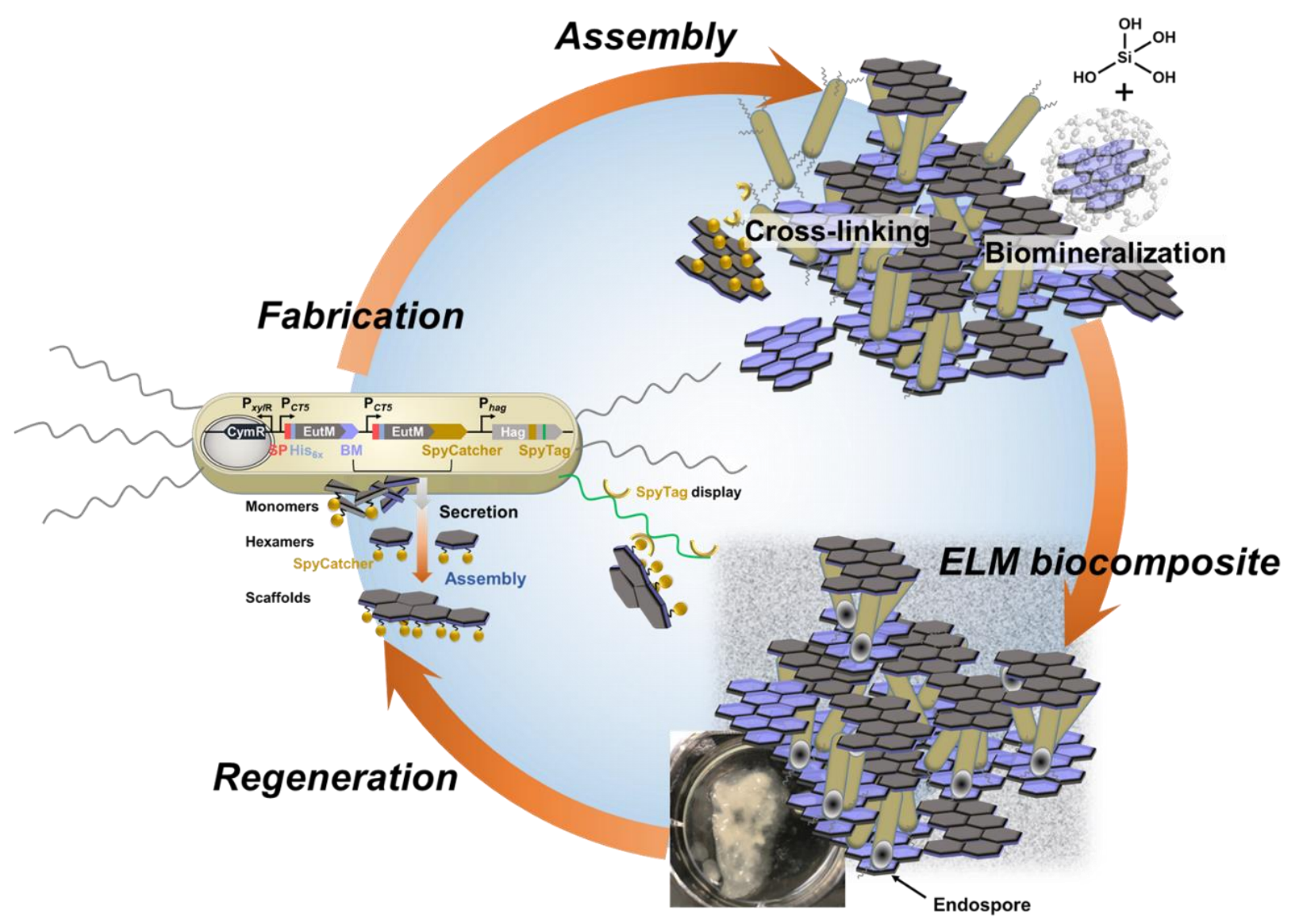

Fig. 1: Formation of a living silica biocomposite material.

B. subtilis is designed to secrete a protein matrix from self-assembling EutM scaffold building blocks. Cells display a SpyTag on engineered clustered, polar flagella for cross-linking with SpyCatcher domains fused to scaffold building blocks. Scaffolds are designed to enhance silica polymerization and cells are modified to retain spores as endospores to create a durable biocomposite material that can be regenerated. (BM, biomineralization peptide; SP, secretion signal peptide). 

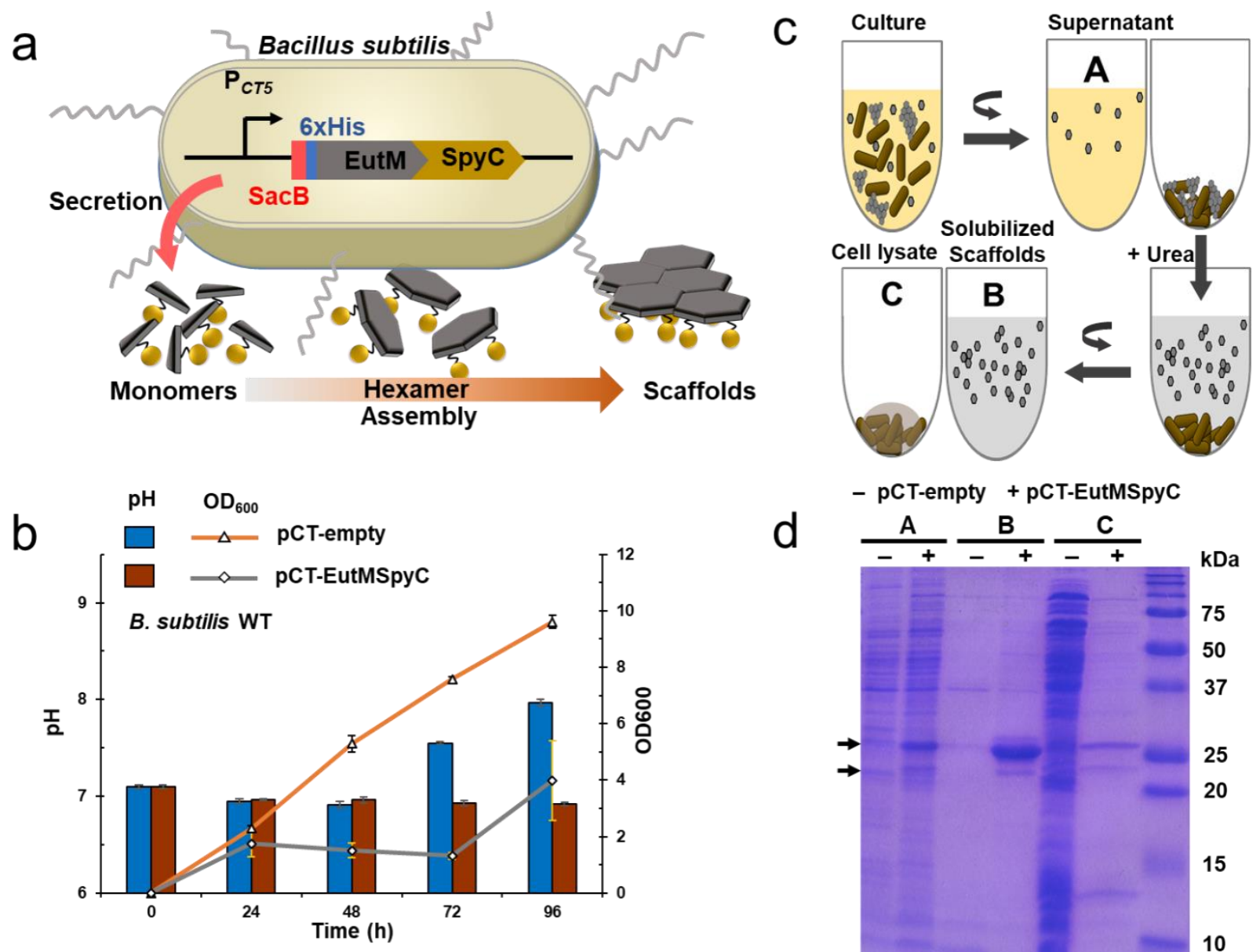

- pCT-empty + pCT-EutMSpyC

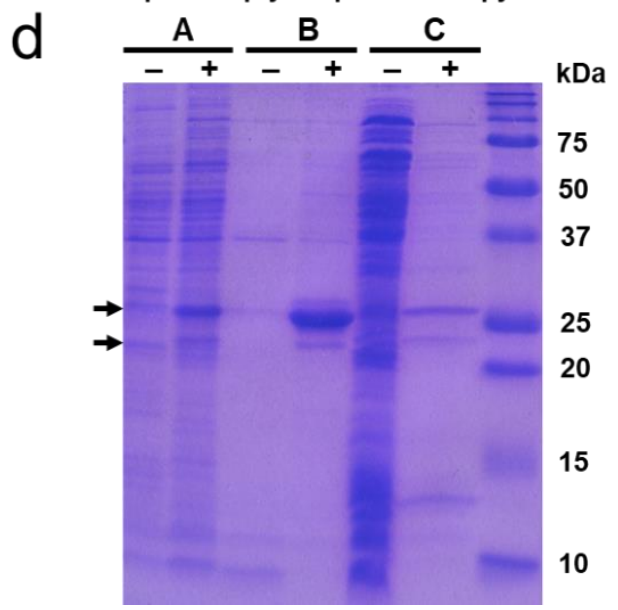

Fig. 2: Establishment of self-assembling scaffold building block secretion.

a Self-assembling EutM scaffold building blocks containing a C-terminal SpyCatcher (SpyC) domain and a SacB secretion signal sequence followed by a His-tag are expressed and secreted by $B$. subtilis under the control of a cumate inducible promoter.

b Optimal secretion of EutM-SpyCatcher scaffold building blocks was obtained in SMM at $20^{\circ} \mathrm{C}$. Protein expression was induced after reaching $\mathrm{OD}_{600}$ of $0.4-0.7$. Growth and $\mathrm{pH}$ of cultures was observed for $96 \mathrm{~h}$ and compared to an empty plasmid control culture. Error bars represent the standard deviations of three biological replicate cultures.

c, d Protein expression and secretion after $48 \mathrm{~h}$ of cultivation were analyzed by SDS-PAGE. Selfassembling scaffolds settle with cells, requiring their solubilization from cell pellets by a gentle wash with 4 M urea (see Methods for details). Samples analyzed include: (A) Culture supernatant (10-fold concentrated by TCA precipitation), (B) Urea supernatant after scaffold solubilization, and (C) resulting cell pellet after lysozyme treatment for analysis of remaining protein. Arrows indicate EutM-SpyCatcher bands. Expected sizes are $24.4 \mathrm{kDa}$ and $21.1 \mathrm{kDa}$ with and without SacB secretion signal peptide, respectively. 
a

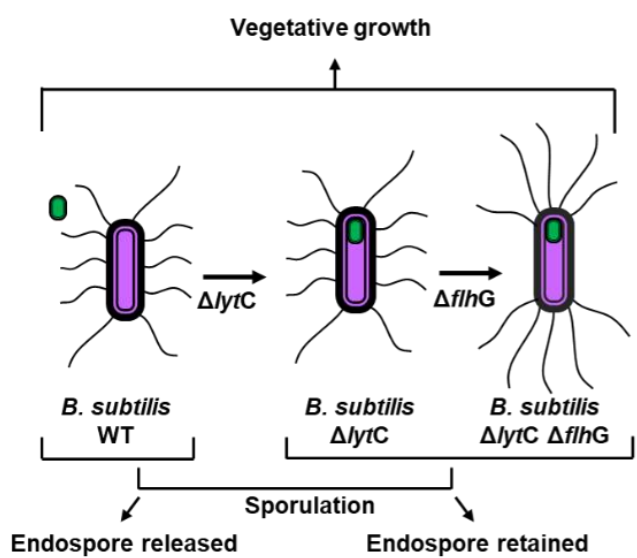

C

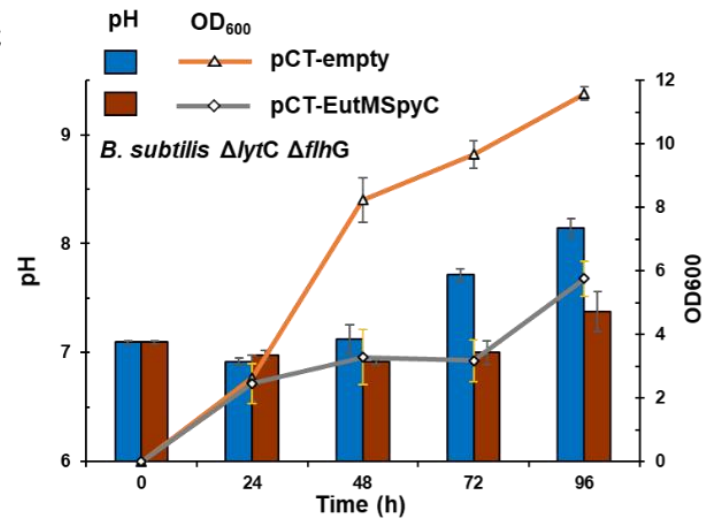

b

B. subtilis WT pCT-EutMSpyC

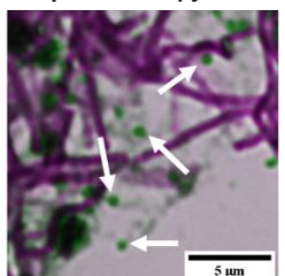

B. subtilis $\Delta$ lytc $\Delta$ flhG pCT-EutMSpyC
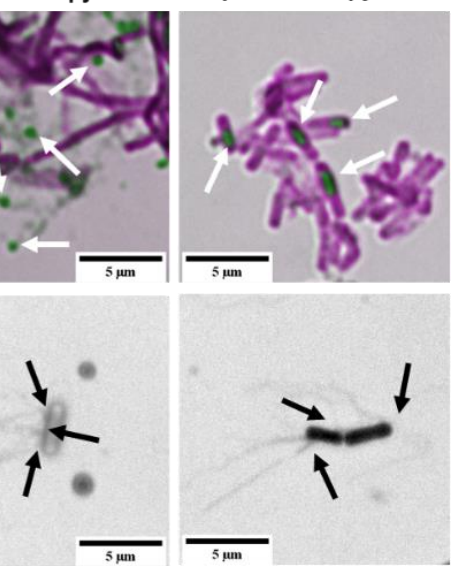

d

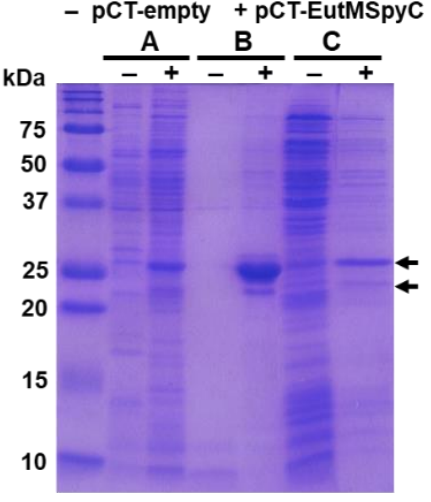

\section{Fig. 3: Development of a Bacillus strain for durable material fabrication.}

a Construction of a $B$. subtilis $\Delta l y t C \Delta f l h G$ strain deficient in spore release and exhibiting polar, clustered flagella was achieved by deleting the autolysin LytC and the flagella basal body protein FlhG.

b Sporulation and flagella phenotypes of $B$. subtilis WT and $B$. subtilis $\Delta / y t C \Delta f l h G$ transformed with pCTEutMSpyC were compared to confirm endospore and polar flagella phenotype of the engineered strain under expression conditions. Representative samples show spore release for the wild-type strain (observed in LB, $48 \mathrm{~h}, 20^{\circ} \mathrm{C}$ shown) and endospore formation by the engineered strain (SMM, $72 \mathrm{~h}, 20^{\circ} \mathrm{C}$ shown) (see also Fig. S2, S3 and S6). Peritrichous flagella are observed for the wild-type strain (LB, $48 \mathrm{~h}, 20^{\circ} \mathrm{C}$ shown) under all tested growth conditions through $72 \mathrm{~h}$ and flagellation decreases after $48 \mathrm{~h}$ (see also Fig. S2 and S3). Clustered polar flagella are observed in the engineered strain that are prevalent through $48 \mathrm{~h}$ of growth (SMM, $48 \mathrm{~h}, 20^{\circ} \mathrm{C}$ shown) (see also Fig. S6). Samples were stained with either malachite green and safranin red (top) or RYU (bottom) and examined by light microscopy at 100x. Spore images had red replaced with magenta and flagella images were converted to grayscale.

c $B$. subtilis $\Delta / y t C \Delta$ flhG strains transformed with the $\mathrm{pCT}$-empty (control) or pCT-EutMSpyC plasmids and cultured for $96 \mathrm{~h}\left(\mathrm{SMM}, 20^{\circ} \mathrm{C}\right)$ show growth characteristics comparable to the $B$. subtilis wild-type strain in Fig. 2. Error bars represent the standard deviations of three biological replicate cultures.

d Expression and secretion of EutM-SpyCatcher by the engineered B. subtilis $\Delta / y t C \Delta f / h G$ strain was verified in SMM medium at $20^{\circ} \mathrm{C}$ after $48 \mathrm{~h}$ of induction by SDS-PAGE. Arrows indicate EutM-SpyCatcher bands that are not present in the empty plasmid control. A strong band corresponding to urea solubilized EutM-SpyCatcher scaffolds (B) from co-pelleted cells confirm comparable protein expression and secretion levels of the engineered and the wild-type strains (Fig. 2). Samples (A) and (C) corresponding to 10-fold concentrated culture supernatant and cell lysate after scaffold solubilization also show comparable protein bands to the wild-type strain. 

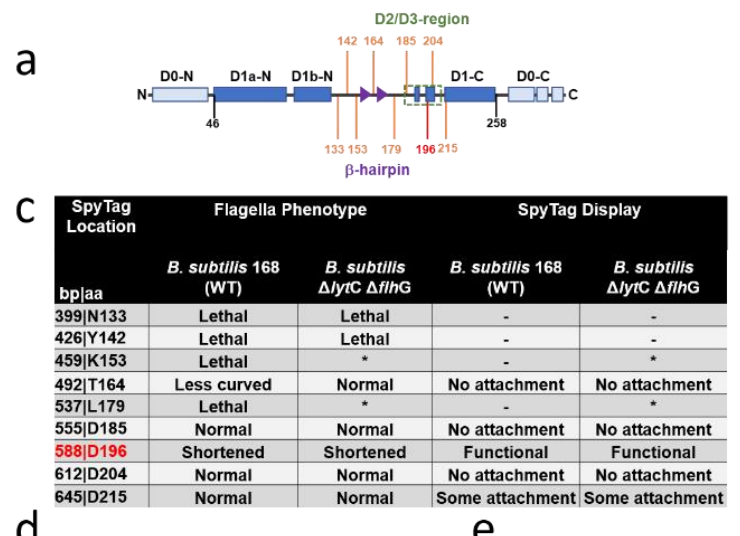

d

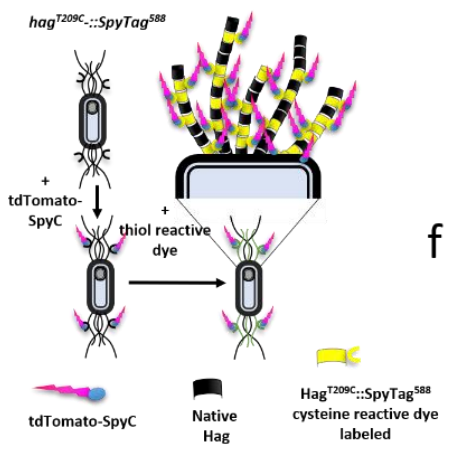

e

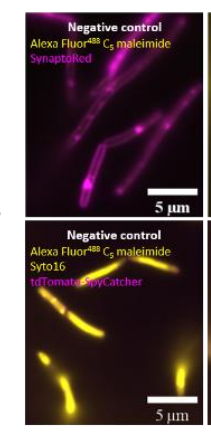

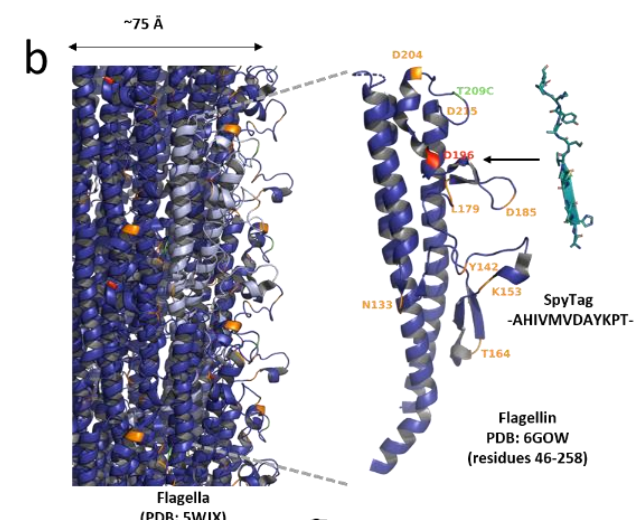

$\mathrm{g}$
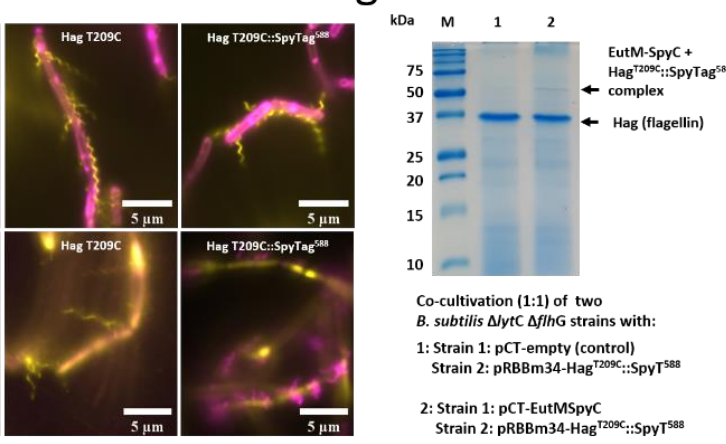

Fig. 4: Engineering of flagella for SpyTag display.

a Flagella of $B$. subtilis were engineered for SpyTag display by inserting the SpyTag sequence at different locations in the hypervariable regions of the flagellin protein (encoded by hag). Shown are conserved domains, including the solvent exposed D2/D3 domains ${ }^{57}$.

b Renderings of the flagellar filament and the flagellin structure ${ }^{72}$ show the locations of the SpyTag insertions (orange, red) and the T209C mutation for staining with a cysteine reactive dye. The functional SpyTag insertion site is highlighted in red. Also shown are the SpyTag structure (PDB: 4MLI) and sequence. c SpyTag insertions into the hag gene had different impacts on flagella phenotypes and SpyTag-display by Bacillus strains expressing plasmid born hag ${ }^{T 209 C}::$ SpyTag ${ }^{x x}$ under the control of its native promoter. Several hag insertion mutants could not be transformed and seem to be lethal when expressed in trans of the genomic hag copy. $\left(^{*}\right)$ Indicates this hag mutant could be transformed into Bacillus but recovered plasmids were mutated. No or some attachment means that modified flagella could not or only be weakly labelled with tdTomato SpyCatcher.

d Flagella assemble from genomic flagellin (hag) and plasmid born, engineered flagellin $\left(\mathrm{Hag}^{\mathrm{T} 209 \mathrm{C}}\right.$ SpyTag $\left.{ }^{\mathrm{Xx}}\right)$ subunits. Engineered flagellins are stained with a cysteine reactive dye (Alexa Fluor ${ }^{\mathrm{TM}}$ $488 \mathrm{C}_{5}$ maleimide) and functional SpyTag display was tested by growing cultures with purified tdTomatoSpyCatcher protein.

e Fluorescence imaging of $B$. subtilis $\Delta / y t C \Delta f / h G$ transformed with pCT-empty, pRBBm34-Hag ${ }^{\top 209 C}$ or

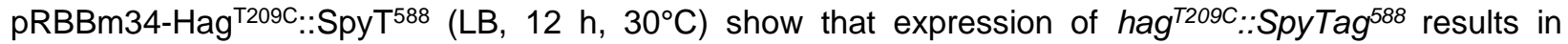
clustered, polar flagella that are shorter than the flagella assembled upon expression of hag ${ }^{T 209 C}$. Cells are stained and images false colored as indicated. See Fig. S9 for other SpyTag positions.

f Functional SpyTag display by Hag ${ }^{\top 209 C}$ ::SpyTag ${ }^{588}$ was confirmed by growing the three strains under the same conditions in the presence of purified tdTomato-SpyCatcher. Cells were stained and images false colored as indicated.

g In situ attachment of secreted EutM-SpyCatcher via isopeptide bond formation with SpyTags displayed on flagella of $B$. subtilis $\Delta / y t C \Delta f / h G$ expressing hag ${ }^{T 209 C:: S p y T a g}{ }^{588}$ was verified by $1: 1$ co-cultivation with strains transformed with pCT-EutMSpyC or pCT-empty (control). After 4 days (SMM, $20^{\circ} \mathrm{C}, 10 \mu \mathrm{M}$ cumate), flagella were extracted and analyzed by SDS-PAGE. A faint band with the expected size of $55 \mathrm{kDa}$ for a EutM-SpyCatcher $(21.1 \mathrm{kDa})$ and Hag ${ }^{\mathrm{T} 209 \mathrm{C}:: S p y T a{ }^{588}}(34.1 \mathrm{kDa})$ protein complex was detected in co- 
bioRxiv preprint doi: https://doi.org/10.1101/2021.08.13.456252; this version posted August 13, 2021. The copyright holder for this preprint (which was not certified by peer review) is the author/funder. All rights reserved. No reuse allowed without permission.

cultures secreting EutM-SpyCatcher. Genome encoded flagellin (32.6 kDa, encoded by hag) appears to be the major protein isolated from both co-cultures. 
a
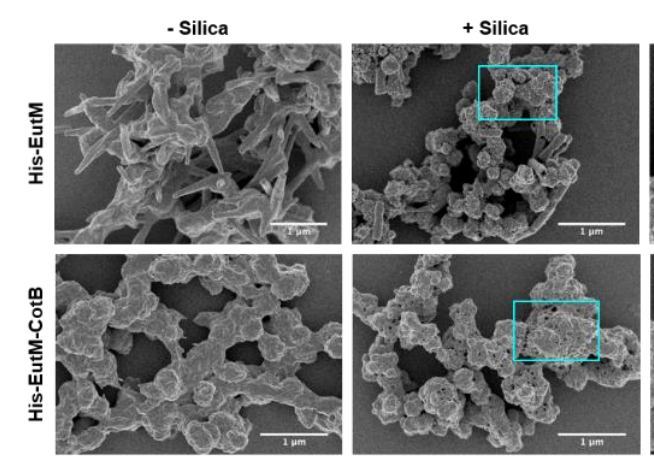

b

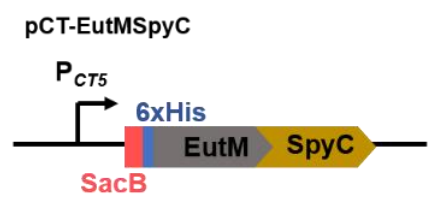

$$
\text { pCT-EutMCotB-EutMSpyc }
$$
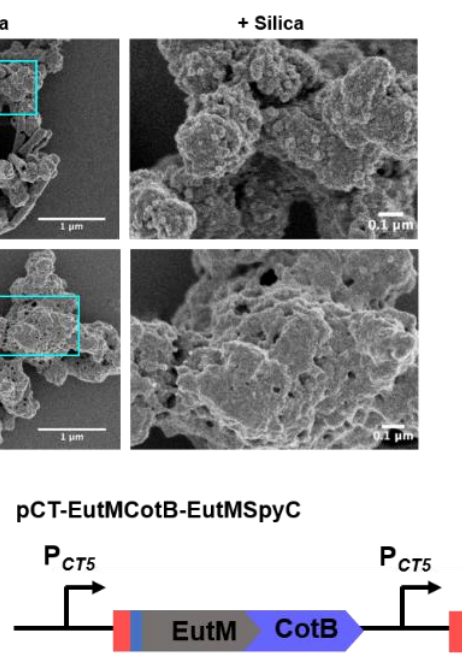

C

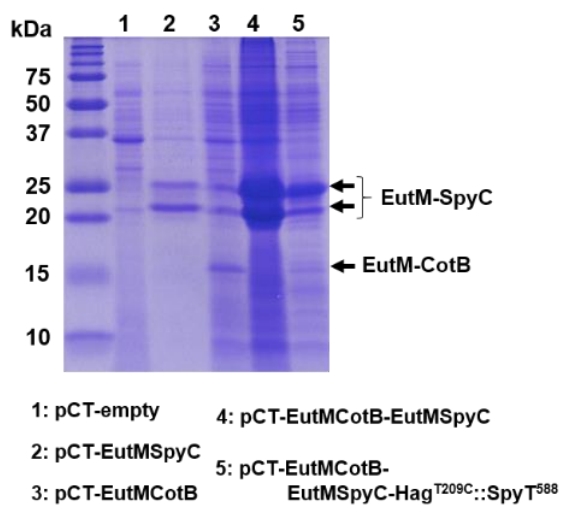

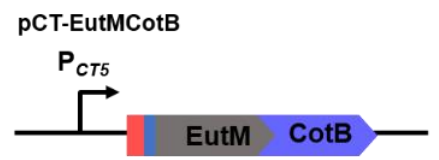

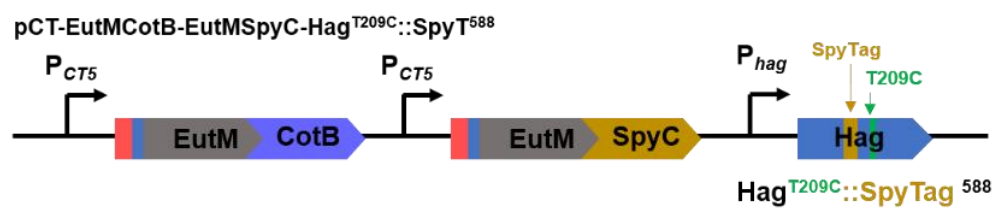

Fig. 5: Implementing silica biomineralization by scaffolds.

a Silica biomineralization by His-EutM and His-EutM-CotB scaffolds expressed and purified from E. coli was investigated by SEM following the incubation of $1 \mathrm{mg} / \mathrm{mL}$ protein with and without $100 \mathrm{mM}$ silica for 2 $\mathrm{h}$ at room temperature. His-EutM formed tubular nanostructures that appeared to be rolled-up planar arrays fused together at multiple locations. His-EutM-CotB formed similar scaffolds but with a greater surface roughness (left column). SEM images show an increase in scaffold surface roughness, surface porosity, and the presence of silica nanoparticles fused to scaffolds which are characteristics of silicification (center and right columns). Silica nanoparticle formation was most prominent on His-EutM suggesting that silicification may have been nucleated in the solution phase by this protein. In contrast, a significant increase in surface roughness seen for His-EutM-CotB scaffolds suggests surface catalyzed biomineralization by these structures.

b Different plasmids were constructed for EutM-CotB expression and secretion in B. subtilis alone or together with EutM-SpyCatcher as shown. For final ELM fabrication, a consolidated plasmid was designed for the co-expression of EutM-SpyCatcher and EutM-CotB with Hag ${ }^{\top 209 C:: S p y T a g} 588$ in B. subtilis $\Delta / y t C$ $\Delta f / h \mathrm{G}$. Each gene is expressed by its own promoter (cumate inducible $\mathrm{P}_{\text {CT5 }}$ promoter, native hag promoter). c Expression and secretion of EutM-CotB by $B$. subtilis $\Delta / y t C \Delta f / h G$ was investigated and compared to EutM-SpyCatcher. Urea solubilized fractions were prepared from cultures transformed with different expression plasmids and grown as in Fig. 2. Except for pCT-EutMSpyC (lane 2), all urea fractions from the different recombinant cultures were concentrated 10-fold by TCA precipitation prior to SDS-PAGE analysis. EutM-CotB is present in the solubilized fractions, but at significantly lower levels than EutM-SpyCatcher. 


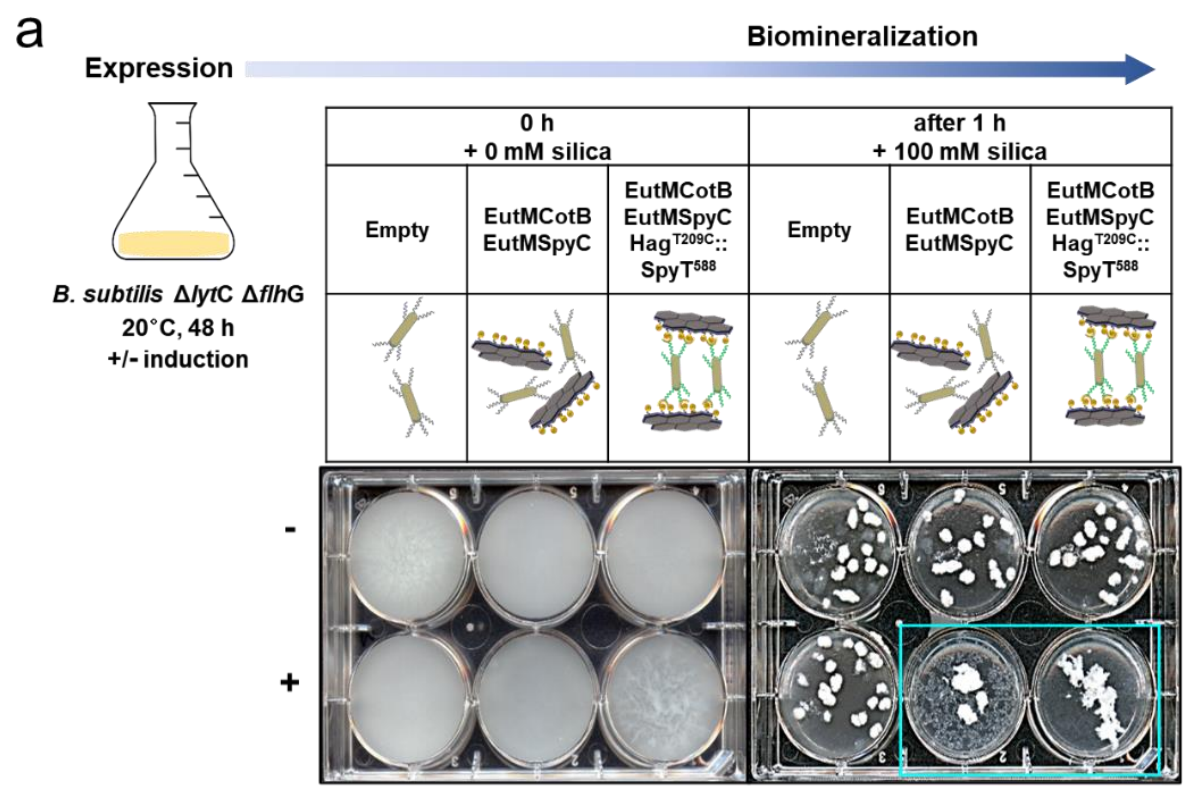

b

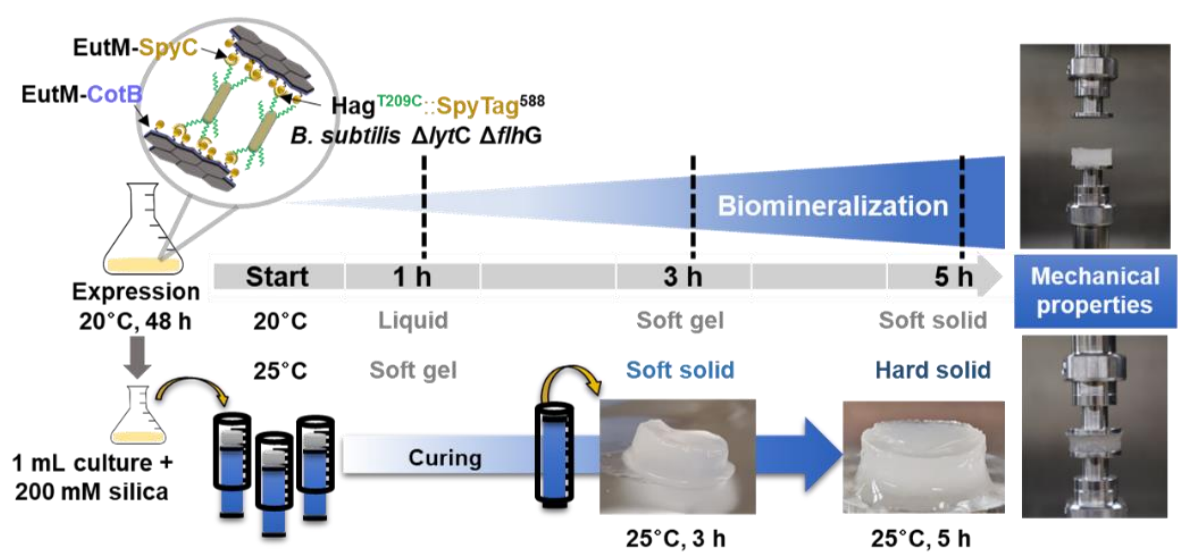

C

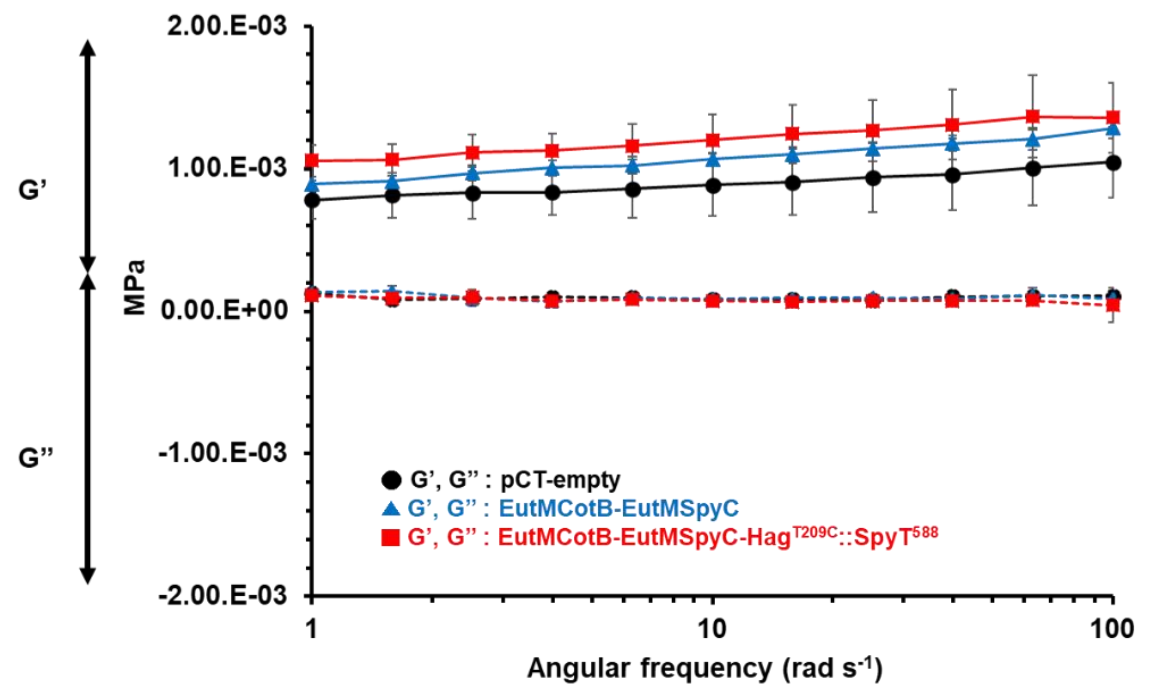




\section{Fig. 6: Formation of living, silica biocomposites with increased rigidity}

a Generation of silica biocomposites was tested by cultivating $B$. subtilis $\Delta l y t C \Delta f l h G$ transformed with plasmids (empty as control) for expression of the shown proteins and their functions. Cultures were first grown $\left(\mathrm{SMM}, 20^{\circ} \mathrm{C}, 48 \mathrm{~h}\right.$ ) with (+ induced) and without (- uninduced) induction of protein expression. $5 \mathrm{~mL}$ cultures were transferred to 6 -well plates $\left(0 \mathrm{~h}, 0 \mathrm{mM}\right.$ silica) and incubated $\left(20^{\circ} \mathrm{C}, 100 \mathrm{rpm}\right)$ for $1 \mathrm{~h}$ after addition of $100 \mathrm{mM}$ silica $(1 \mathrm{~h}, 100 \mathrm{mM})$. Only induced cultures that co-expressed EutM-CotB and EutMSpyCatcher formed aggregated silica material upon induction. The aggregation appears to be more pronounced in cultures that also co-expressed SpyTag labeled flagella for cross-linking of cells and EutM scaffolds. Experiments were performed with three biological culture replicates (see Fig. S13).

b 3D blocks of silica biocomposite materials were then fabricated from cultures of the same three strains grown under the same conditions with induction. After $48 \mathrm{~h}$ of growth, $200 \mathrm{mM}$ silica was added into $1 \mathrm{~mL}$ cultures in syringes as molds and the aliquots of the cultures were cured at $20^{\circ} \mathrm{C}$ or $25^{\circ} \mathrm{C}$. At different time points, silica gel plugs were removed from their molds to evaluate their hardness. After $5 \mathrm{~h}$ of curing at $25^{\circ} \mathrm{C}$, solid gel plugs suitable for rheology testing were obtained.

c Rheological properties of the cured gel plugs were measured using an extensional DMA Rheometer with $15 \mathrm{~mm}$ compression disks. A frequency sweep was performed with a gap of $4.5 \mathrm{~mm}$ and an oscillation strain of $1 \%$. Three biological replicate gel blocks were measured for each strain. Variation was observed in the storage modulus ( $G^{\prime}$, solid line) and loss modulus (G', dashed line). The engineered strain, EutMCotB-EutMSpyC-Hag ${ }^{\top 209 C}:: S p y T^{588}$ provided the greatest cross-linking effect, resulting in a greater force required to deform the material and increased rigidity compared to the empty plasmid control. 
a

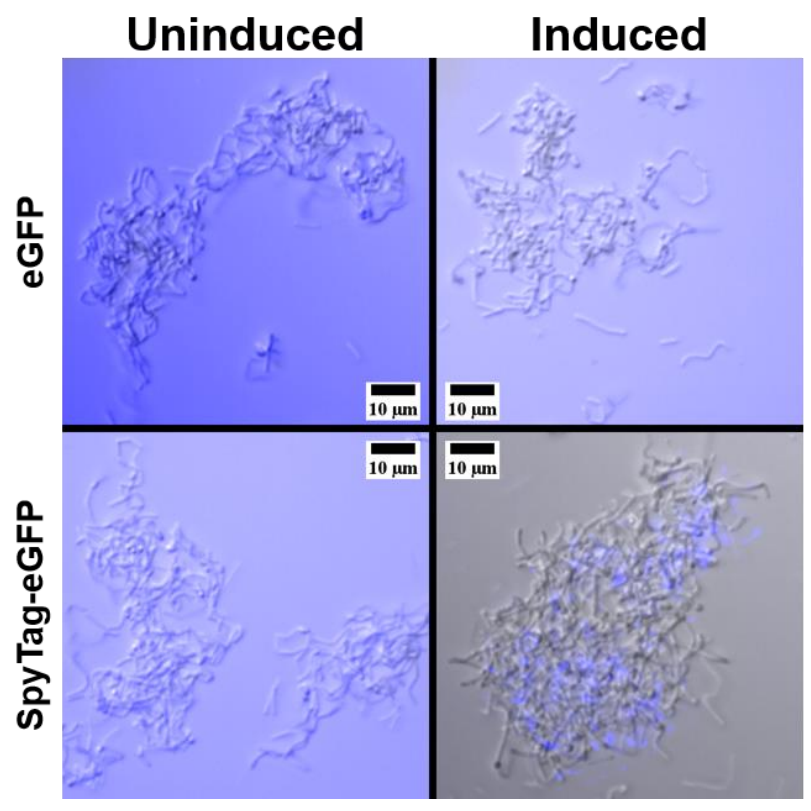

b

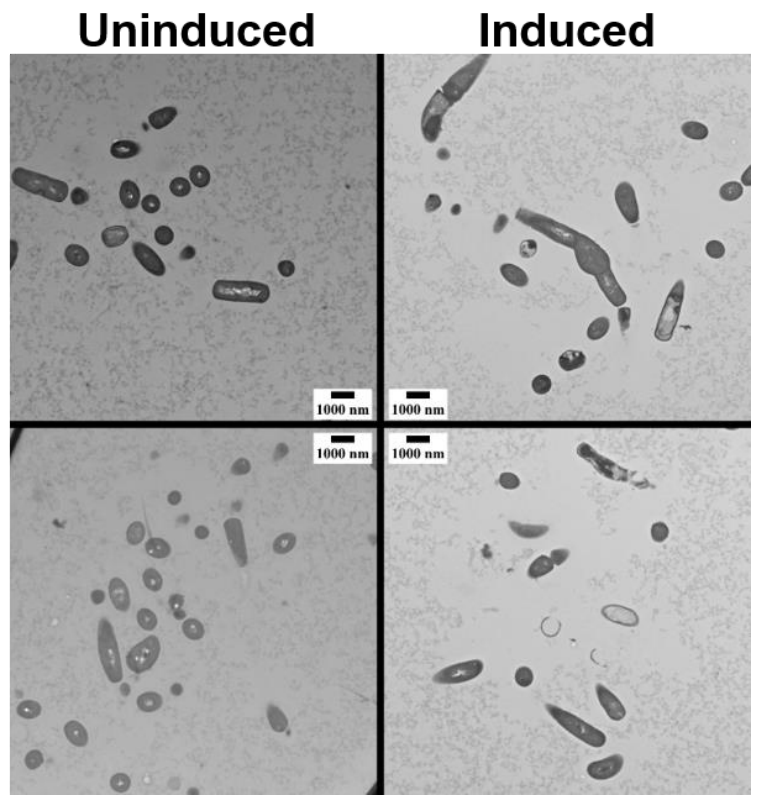

Fig. 7: Microscopy characterization of Bacillus silica biocomposites.

a The location of assembled scaffolds was tested by cultivating $B$. subtilis $\Delta l y t C \Delta f l h G$ transformed with EutMCotB-EutMSpyC-Hag ${ }^{T 209 C}:: S p y T^{588}$ plasmid (uninduced as control). Cultures were first grown (SMM, $20^{\circ} \mathrm{C}, 48 \mathrm{~h}$ ) with (induced) and without (uninduced) cumate induction of protein expression. $500 \mu \mathrm{L}$ of culture was mixed with $0.125 \mathrm{mg}$ eGFP or SpyTag-eGFP and mixed at RT for 30 minutes before acquiring DIC and GFP images which were merged and GFP channel colored blue. Induced cultures have EutM scaffolds assembled outside and between cells, with available binding sites for additional SpyTagged proteins.

b 3D blocks of silica biocomposite materials were then formed from cultures of the same sample examined in a. $200 \mathrm{mM}$ silica was added after $48 \mathrm{~h}$ of growth and $1 \mathrm{~mL}$ aliquots of the cultures were transferred to syringes as molds for curing at $25^{\circ} \mathrm{C}$. After $5 \mathrm{~h}$ of curing at $25^{\circ} \mathrm{C}$, solid gel plugs were cut into $2 \mathrm{~mm}^{3}$ pieces and prepared for thin sectioning. Cells were observed in clusters for both the uninduced and induced cultures, however only the induced culture contained clear zones around cells. This zone corresponds to the SpyTag-eGFP labeled areas above and indicates there is interference with staining the scaffolds after silica is biomineralized. 

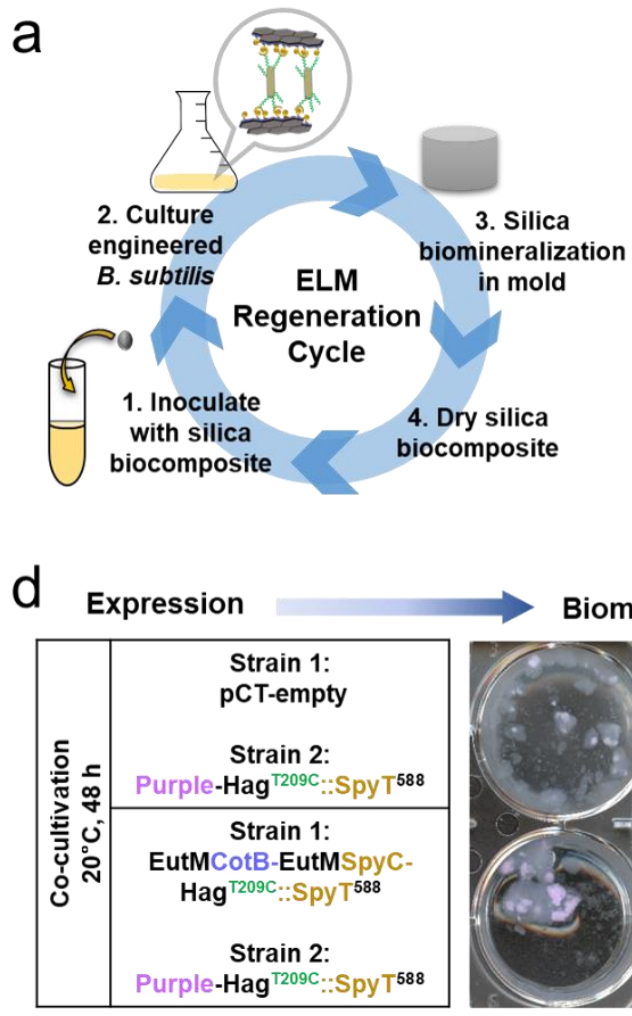

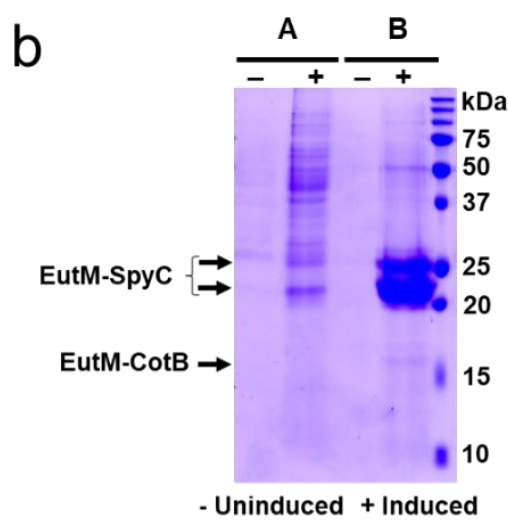

- Uninduced + Induced

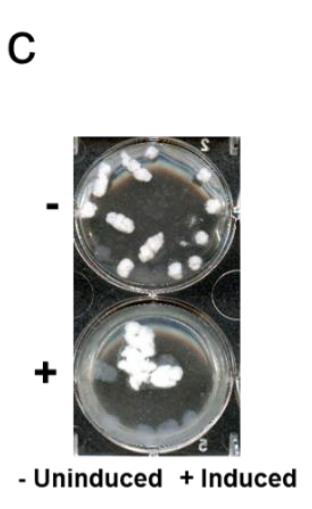

e

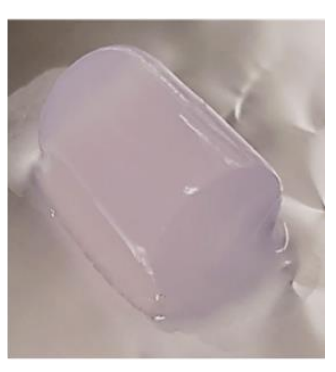

Fig. 8: ELM biocomposite regeneration and proof-of-concept for material functionalization.

a Silica biocomposite material regeneration was tested by re-inoculating cultures with a piece of a silica plug fabricated from $B$. subtilis $\triangle l y t C \Delta f / h G$ cultures co-expressing EutM-CotB and EutM-SpyCatcher and $\mathrm{Hag}^{\mathrm{T} 209 \mathrm{C}}$ SpyTag ${ }^{588}$, biomineralized with $200 \mathrm{mM}$ silica (see Fig. 6) and cured for $24 \mathrm{~h}$ at $25^{\circ} \mathrm{C}$. Cultures were regrown $\left(\mathrm{SMM}, 20^{\circ} \mathrm{C}, 48 \mathrm{~h}\right.$ ) with (+ induced) and without (- uninduced) cumate induction of protein expression.

b Expression and secretion of EutM scaffold building blocks by the induced, regenerated cultures was confirmed by SDS-PAGE analysis of culture supernatant (A) and urea solubilized scaffolds (B). All fractions were concentrated 10-fold by TCA precipitation prior to SDS-PAGE analysis.

c Silica biomineralization into a cross-linked biocomposite material was confirmed for the induced, regenerated cultures after $1 \mathrm{~h}$ at $20^{\circ} \mathrm{C}$ with $100 \mathrm{mM}$ silica in 6-well plates as in Fig. 6.

d Proof-of-concept incorporation of additional functions into the silica ELM was demonstrated by the cocultivation of two strains, one strain co-expresses scaffold building blocks and SpyTagged flagellin, while a second strain co-expresses a purple chromophore protein and SpyTagged flagellin. In the control coculture, the plasmid for scaffold building block secretion in one strain was replaces with an empty plasmid. Strains were inoculated at a 1:1 ratio and cultures grown under inducing conditions for biomineralization as described above. Only co-cultures that co-expressed EutM-CotB and EutM-SpyCatcher formed an aggregated silica material with embedded purple cells.

e A block of a purple, silica biocomposite $(15 \mathrm{~mm} \times 30 \mathrm{~mm})$ was then fabricated from co-cultures of engineered strains co-expressing scaffold building blocks and the purple protein together with SpyTagged flagellin. A solid material was formed with $200 \mathrm{mM}$ silica after curing for $24 \mathrm{~h}$ at $25^{\circ} \mathrm{C}$. 


\section{SUPPLEMENTARY INFORMATION}

Engineering Bacillus subtilis for the formation of a durable living biocomposite material

Sun-Young Kang ${ }^{1,3^{*}}$, Anaya Pokhrel ${ }^{1,3^{*}}$, Sara Bratsch ${ }^{1,3^{*}}$, Joey J. Benson ${ }^{2}$, Seung-Oh Seo ${ }^{1,3}$, Maureen B. Quin ${ }^{1,3}$, Alptekin Aksan ${ }^{2,3}$, Claudia Schmidt-Dannert ${ }^{1,3^{* *}}$

${ }^{1}$ Department of Biochemistry, Molecular Biology \& Biochemistry, University of Minnesota, Minneapolis, MN 55455, USA

${ }^{2}$ Department of Mechanical Engineering, University of Minnesota, Minneapolis, MN 55455, USA

${ }^{3}$ BioTechnology Institute, University of Minnesota, St. Paul, MN 55108, USA

* These authors contributed equally to this work.

** Corresponding author:

Prof. Claudia Schmidt-Dannert

140 Gortner Laboratory

University of Minnesota

1479 Gortner Avenue

St. Paul, MN 55108

E-mail: schmi232@umn.edu

Phone: +1 6126255782 


\section{TABLE OF CONTENTS}

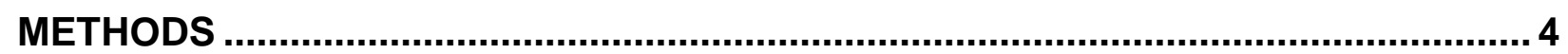

1. Materials

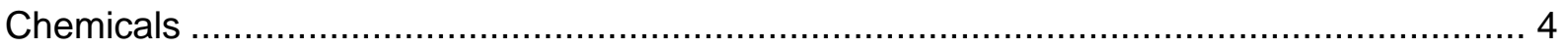

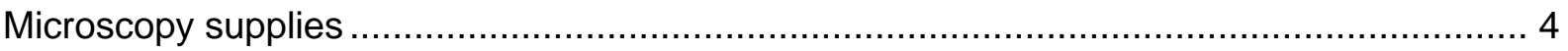

Protein purification

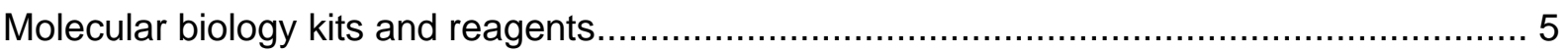

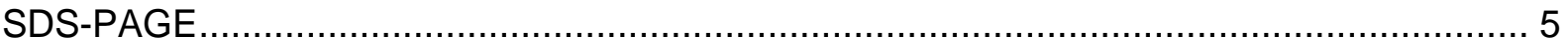

2. Bacterial strains, media and general molecular biology methods............................ 5

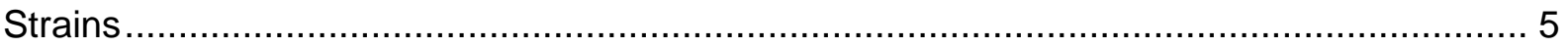

Media

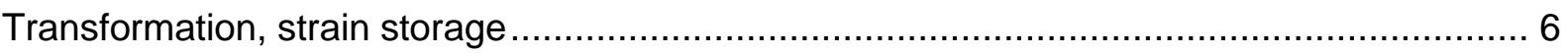

3. Construction of plasmids and in-frame deletion in $B$. subtilis................................. 6

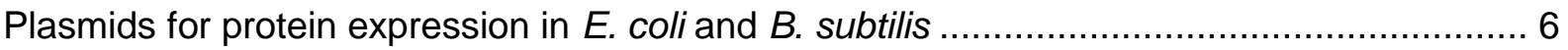

Plasmids for SpyTag display on B. subtilis flagella .................................................... 7

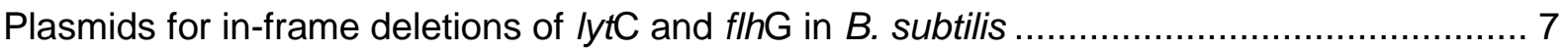

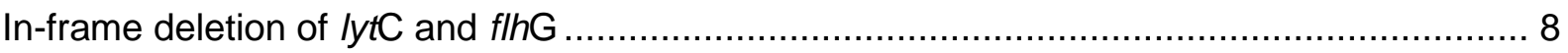

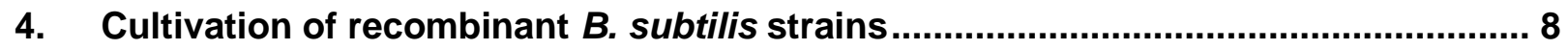

5. Analysis of scaffold building block expression and secretion by Bacillus strains.. 9

6. De novo sequencing of secreted EutM-SpyCatcher proteins .................................10

7. Analysis of EutM-SpyCatcher attachment to Hag $^{\text {T209C }:: S p y T a g}{ }^{588}$ flagella ................10

8. Protein purification................................................................................................11

9. Silica biomineralization and material characterization ..........................................12

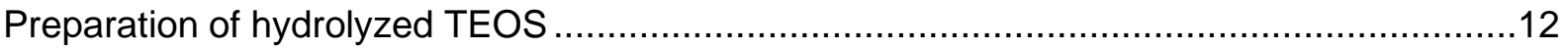

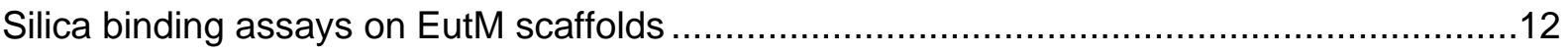

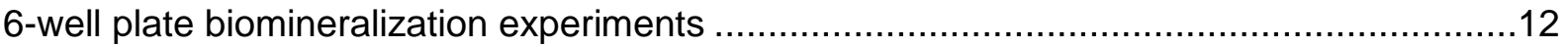

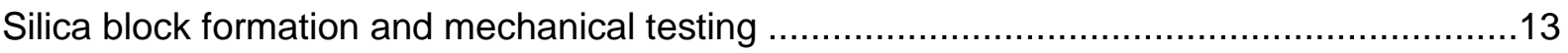

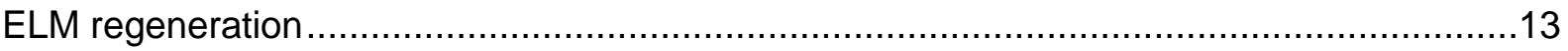

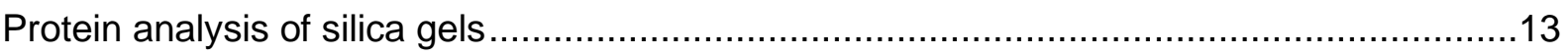

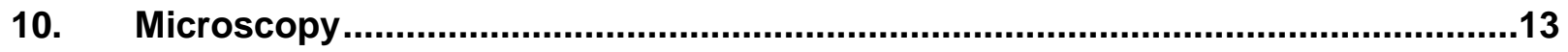

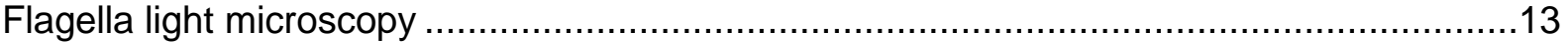

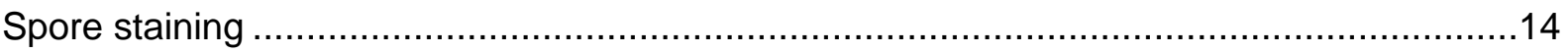

SEM imaging

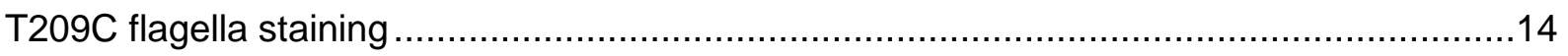

In situ labeling of SpyTagged flagella with His-tdTomato-SpyCatcher ..............................15

TEM of purified scaffolds from E. coli and Bacillus ..................................................15

In situ labeling of secreted EutM-SpyCatcher scaffolds with His-SpyTag-eGFP ...................16

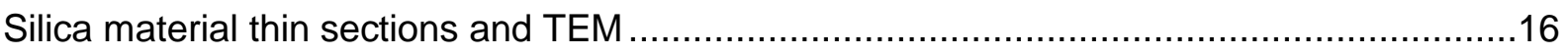

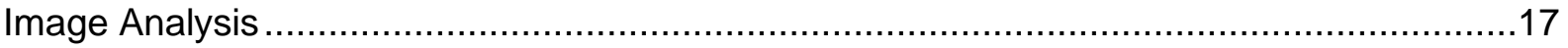


SUPPORTING FIGURES.

Fig. S1: Establishing growth conditions suitable for silica biocomposite formation with $B$. subtilis pCT-empty and pCT-EutMSpyC.

Fig. S2: Analysis of spore formation and flagella phenotypes of $B$. subtilis pCT-empty under different growth conditions.

Fig. S3: Analysis of spore formation and flagella phenotypes of B. subtilis pCT-EutMSpyC. 20

Fig. S4: Testing of additional secretion signal sequence for scaffold building block secretion by $B$. subtilis.

Fig. S5: Experimental workflow used for the analysis of EutM scaffold building block secretion and expression by engineered $B$. subtilis.

Fig. S6: Construction and phenotypical characterization of the double deletion strain $B$.

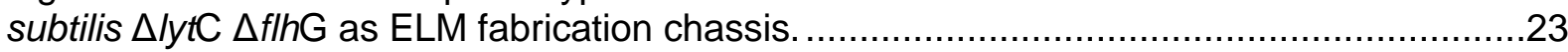

Fig. S7: Analysis of EutM-SpyCatcher secreted by B. subtilis $\Delta / y t C \quad \Delta f l h G$. ......................24

Fig. S8: Comparison of scaffold formation by EutM-SpyCatcher purified from recombinant $E$. coli and $B$. subtilis $\Delta / y t C \Delta f / h G$ cultures. .25

Fig. S10: Characterization of silica binding and precipitation by EutM biomineralization peptide fusion proteins.

Fig. S11: Comparison and optimization of silica gel formation by $B$. subtilis WT cultures expressing EutM-CotB scaffolds or no scaffolds.

Fig. S12: Visualization of cell phenotypes of $B$. subtilis $\Delta / y t C \Delta f l h G$ cultures transformed with different plasmids. .30

Fig. S13: Silica biomineralization by engineered $B$. subtilis $\Delta / y t C \Delta f / h G$ cultures. .................31

Fig. S14: Additional characterization of Bacillus silica biocomposites. ...............................32

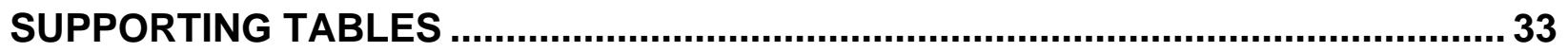

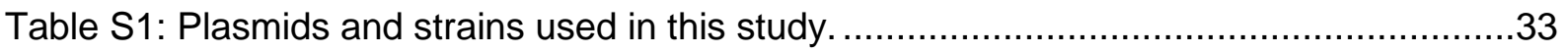

Table S2: Amino acid sequences of proteins and peptides in this study...........................36

Table S3: Nucleotides sequences of proteins and peptides in this study...........................39

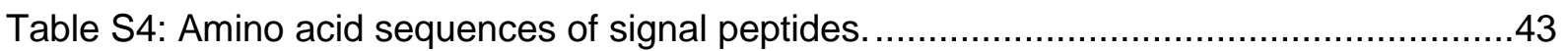

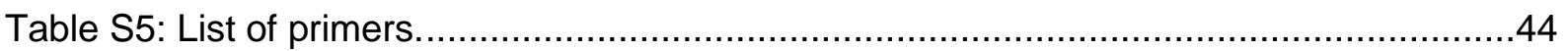

Table S6: Variation of storage modulus ( $\left.G^{\prime}\right)$ and loss modulus (G') with frequency of silica gels.

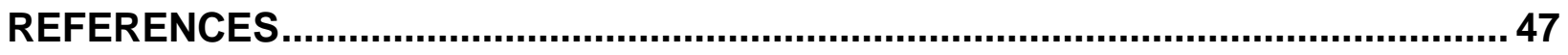




\section{METHODS}

\section{Materials}

\section{Chemicals}

Reagent grade tetraethyl orthosilicate (TEOS), ammonium molybdate tetrahydrate, 4methylaminophenol sulphate, anhydrous sodium sulfite, all acids and bases, and other chemicals were purchased from Sigma-Aldrich (Sigma-Aldrich Corp., MO, USA).

\section{Microscopy supplies}

Remel RYU flagella stain, polysine microscope slides, plain precleaned glass microscope slides, Alexa Fluor ${ }^{\mathrm{TM}} 488 \quad \mathrm{C}_{5}$ maleimide, Syto16, and Trump's Fixative were purchased from ThermoFisher Scientific (Waltham, MA, USA). $22 \mathrm{~mm} 1.5$ cover glasses were purchased from Azer Scientific (Morgantown, PA, USA). 200 mesh copper grids with $10 \mathrm{~nm}$ formvar and $1 \mathrm{~nm}$ carbon, aqueous $2 \%$ uranyl acetate, and EM grade: $10 \%$ glutaraldehyde, $0.2 \mathrm{M}$ sodium cacodylate trihydrate $\mathrm{pH} 7.4,16 \%$ paraformaldehyde, and $4 \%$ osmium tetraoxide were purchased from VWR International, an Electron Microscopy Sciences reseller (Radnor, Pennsylvania, USA). SynaptoRed ${ }^{\mathrm{TM}} \mathrm{C} 2$ was purchased from Avantor delivered by VWR a Biotium reseller (Radnor, PA, USA). FluoroShield was used in fluorescent microscopy experiments and was purchased from AbCam (Cambridge, MA, USA). Silicon wafers were used for SEM and obtained from Ted Pella (Reeding, CA, USA). Schaeffer and Fulton Spore stain kit (containing malachite green 50 $\mathrm{g} / \mathrm{L}$ and safranin $\mathrm{O} 5 \mathrm{~g} / \mathrm{L}$ ) was obtained from MilliporeSigma (Burlington, MA, USA).

\section{Protein purification}

Spectra/Por Dialysis Tubing (MWCO 6-8 kDa) used for dialysis of proteins was purchased from Spectrum LifeSciences (Rancho Dominguez, CA, USA). HisTrap ${ }^{\text {TM }}$ FF columns (GE Healthcare, IL, USA) were used for all protein purifications except for proteins used for TEM that were purified from E. coli and secreted proteins from Bacillus that were purified using TALON metal affinity resin from TaKaRa Bio USA, Inc. (Mountain View, CA, USA). $2 \mathrm{~mL}$ of TALON metal affinity resin was used in a Batch/Gravity flow protein purification protocol following manufacturer's directions. Pierce ${ }^{\mathrm{TM}}$ BCA Protein Assay Kit used for protein concentration measurements was purchased from ThermoFisher Scientific (Waltham, MA, USA). Ultrapure water was prepared by filtering deionized water though a Milli-Q water purification system (Millipore, Billerica, MA, USA) to reach a final electrical resistance higher than $18.2 \mathrm{M} \Omega \mathrm{cm}^{-1}$. 


\section{Molecular biology kits and reagents}

All supplies were purchased from New England Biolabs (NEB) (Ipswhich, MA, USA). Q5® HighFidelity DNA polymerase was used for PCR amplifications. The Q5® Site-Directed Mutagenesis kit was used for site directed mutagenesis and the NEBuilder® HiFi DNA Assembly Master Mix was used for Gibson assembly of DNA fragments, and restriction enzymes (Bg/l, BamHI, and Pstl) and T4 DNA Ligase were used for general cloning. Primers were ordered from Integrated DNA Technologies (Coralville, IA, USA).

\section{SDS-PAGE}

40\% acrylamide and bis-acrylamide solution (37.5:1), TEMED and Precision plus all blue prestained protein standard (catalog\# 161-0373) were purchased from Bio-Rad (Hercules, CA, USA). Other chemicals for SDS-PAGE analysis and Coomassie brilliant blue staining were purchased from Sigma-Aldrich.

\section{Bacterial strains, media and general molecular biology methods}

\section{Strains}

Escherichia coli TOP10 (Invitrogen, Carlsbad, CA, USA) was used for cloning and plasmid propagation. E. coli T7 Express (C2566) (NEB) and used for protein expression and purification. Bacillus subtilis 168 (ATCC 23857) was used as a host strain (referred to as WT strain). All plasmids and strains used in this study are listed in Table S1.

\section{Media}

LB (Luria broth; Tryptone $10 \mathrm{~g} / \mathrm{L}, \mathrm{NaCl} 5 \mathrm{~g} / \mathrm{L}$, Yeast extract $5 \mathrm{~g} / \mathrm{L}$ ) medium was used for growth of E. coli and $B$. subtilis strains.

B. subtilis strains were also grown in modified Spizizen's minimal medium (SMM) ${ }^{7,8}$, containing, per liter: $\mathrm{K}_{2} \mathrm{HPO}_{4}-17.5 \mathrm{~g}, \mathrm{KH}_{2} \mathrm{PO}_{4}-7.5 \mathrm{~g}, \mathrm{Na}_{3}$-Citrate $2 \mathrm{H}_{2} \mathrm{O}-1.25 \mathrm{~g}, \mathrm{MgSO}_{4} 7 \mathrm{H}_{2} \mathrm{O}-0.25 \mathrm{~g}, \mathrm{~N}-$ source (L-glutamine - $\left.10 \mathrm{~g},\left(\mathrm{NH}_{4}\right)_{2} \mathrm{SO}_{4}-0.5 \mathrm{~g}\right)$, trace elements $\left(\mathrm{CaCl}_{2} 2 \mathrm{H}_{2} \mathrm{O}-7.35 \mathrm{mg}, \mathrm{MnCl}_{2} 4\right.$ $\mathrm{H}_{2} \mathrm{O}-1 \mathrm{mg}, \mathrm{ZnCl}_{2}-1.7 \mathrm{mg}, \mathrm{CuCl}_{2} 6 \mathrm{H}_{2} \mathrm{O}-0.43 \mathrm{mg}, \mathrm{CoCl}_{2} 6 \mathrm{H}_{2} \mathrm{O}-0.6 \mathrm{mg}, \mathrm{Na}_{2} \mathrm{MoO}_{4} 2 \mathrm{H}_{2} \mathrm{O}-0.6$ $\mathrm{mg})$, Fe-Citrate $\left(\mathrm{FeCl}_{3} 6 \mathrm{H}_{2} \mathrm{O}-1.35 \mathrm{mg}, \mathrm{Na}_{3}\right.$-Citrate $\left.3 \mathrm{H}_{2} \mathrm{O}-10 \mathrm{mg}\right)$, glucose - $5 \mathrm{~g}$, tryptophan $50 \mathrm{mg}$.

SMM was prepared by mixing stock solutions which included, $5 \mathrm{X}$ SMM base $\left(\mathrm{K}_{2} \mathrm{HPO}_{4}-87.5 \mathrm{~g} / \mathrm{L}\right.$, $\mathrm{KH}_{2} \mathrm{PO}_{4}-37.5 \mathrm{~g} / \mathrm{L}, \mathrm{Na}_{3}-$ Citrate $2 \mathrm{H}_{2} \mathrm{O}-6.25 \mathrm{~g} / \mathrm{L}, \mathrm{MgSO}_{4} \cdot 7 \mathrm{H}_{2} \mathrm{O}-1.25 \mathrm{~g} / \mathrm{L}$ ), N-source (sterilized using a $0.2 \mu \mathrm{m}$ filter) (glutamine $\left.-40 \mathrm{~g} / \mathrm{L},\left(\mathrm{NH}_{4}\right)_{2} \mathrm{SO}_{4}-2 \mathrm{~g} / \mathrm{L}\right), 100 \mathrm{X}$ trace elements $\left(\mathrm{CaCl}_{2} \cdot 2 \mathrm{H}_{2} \mathrm{O}-\right.$ $0.735 \mathrm{~g} / \mathrm{L}, \mathrm{MnCl}_{2} \cdot 4 \mathrm{H}_{2} \mathrm{O}-0.1 \mathrm{~g} / \mathrm{L}, \mathrm{ZnCl}_{2}-0.17 \mathrm{~g} / \mathrm{L}, \mathrm{CuCl}_{2} \cdot 6 \mathrm{H}_{2} \mathrm{O}-0.043 \mathrm{~g} / \mathrm{L}, \mathrm{CoCl}_{2} \cdot 6 \mathrm{H}_{2} \mathrm{O}-0.06 \mathrm{~g} / \mathrm{L}$, $\left.\mathrm{Na}_{2} \mathrm{MoO}_{4} \cdot 2 \mathrm{H}_{2} \mathrm{O}-0.06 \mathrm{~g} / \mathrm{L}\right), 100 X$ Fe-citrate (sterilized using a $0.2 \mu \mathrm{m}$ filter) $\left(\mathrm{FeCl}_{3} 6 \mathrm{H}_{2} \mathrm{O}-0.135\right.$ 
$\mathrm{g} / \mathrm{L}, \mathrm{Na}_{3}$-Citrate. $3 \mathrm{H}_{2} \mathrm{O}-1 \mathrm{~g} / \mathrm{L}$ ), $50 \%$ glucose $(500 \mathrm{~g} / \mathrm{L}$ ) and tryptophan (sterilized using a $0.2 \mu \mathrm{m}$ filter) $(5 \mathrm{~g} / \mathrm{L})$.

Fresh solution of sterile $\mathrm{N}$-source and glucose were prepared every time while stocks of other solutions were stored at $4^{\circ} \mathrm{C}$. Working solution of SMM was prepared by mixing $200 \mathrm{~mL}$ of $5 \mathrm{X}$ SMM, $10 \mathrm{~mL}$ of $100 \mathrm{X}$ trace element, $10 \mathrm{~mL}$ of $100 \mathrm{X}$ Fe-Citrate, $10 \mathrm{~mL}$ of $50 \%$ glucose, $10 \mathrm{~mL}$ from $5 \mathrm{mg} / \mathrm{mL}$ stock of Tryptophan and $250 \mathrm{~mL}$ of $\mathrm{N}$-source into $510 \mathrm{~mL}$ of sterile water.

SM1 and SM2 media as described previously by Bennallack et. al., $2014^{9}$ were used for the preparation of $B$. subtilis competent cells.

For plasmid maintenance, LB was supplemented with $100 \mu \mathrm{g} / \mathrm{mL}$ ampicillin for $E$. coli strains while LB and SMM were supplemented with $10 \mu \mathrm{g} / \mathrm{mL}$ tetracycline or $20 \mu \mathrm{g} / \mathrm{mL}$ erythromycin for Bacillus strains.

\section{Transformation, strain storage}

Transformation of $E$. coli with plasmids followed standard molecular biology techniques. Transformation of $B$. subtilis strains was done using a method previously described by Bennallack et. al., 2014 ${ }^{9}$. Transformants were screened by colony PCR using a method modified from Jamal et. al., $2017^{10}$. To prepare samples for colony PCR, a single colony of $B$. subtilis was mixed in $300 \mu \mathrm{L}$ sterile water. Cells were spun down $(21,130 \times g)$ and $200 \mu \mathrm{L}$ of the supernatant was removed. Cells were resuspended in the remaining $100 \mu \mathrm{L}$ of sterile water followed by 20 mins of sonication in a cold-water bath sonicator (Bransonic 3510R-MTH, CT, USA). After sonication, cells were spun down as above and $2 \mu \mathrm{L}$ of the supernatant was used as a template for a $10 \mu \mathrm{L}$ PCR reaction. Plasmids from positive $B$. subtilis transformants were isolated and their sequences verified by Sanger sequencing. Recombinant strains with verified plasmid sequences were stored as glycerol stocks and used in this work.

\section{Construction of plasmids and in-frame deletion in B. subtilis}

All plasmids and strains used in this study are described in Table S1. Plasmid sequences were verified by Sanger sequencing (ACGT, Inc. Wheeling, IL, USA). Amino acid sequences and nucleotide sequences for all proteins and genes reported in this work are found in Table $\mathbf{S 2}$ and Table S3. Primer pairs for Bacillus genomic manipulations and flagellin engineering are listed in Table S5.

\section{Plasmids for protein expression in E. coli and B. subtilis}

Plasmids were constructed using a combination of Gibson Assembly ( $\mathrm{HiFi}$ DNA assembly kit, NEB) for fragment assembly, site directed mutagenesis (Q5® mutagenesis kit, NEB) for shorter 
insertions, deletions and insertions, or restriction enzyme cloning and ligation for larger fragment assembly following manufacturers' instructions and as described previously ${ }^{1,11}$.

Briefly, for the C-terminal fusion of biomineralization peptides to His-EutM in PCT5-His-EutM ${ }^{1,11}$, primers were designed to insert the corresponding into the plasmid using the Q5® mutagenesis kit according to the manufacturers' instructions.

To construct the pCT-His-dtTomato-SpyCatcher plasmid, His-EutM was combined with the fluorescent protein in a previously constructed pCT5-His-EutM-SpyC plasmid ${ }^{2}$ by HiFi Assembly. His-tdTomato-SpyCatcher was amplified from tdTomato-pBAD ${ }^{12}$.

To create the EutM Bacillus expression vectors, His-EutM-SpyCatcher was amplified from pCT5His-EutM-SpyC and inserted into our in-house, cumate inducible Bacillus expression vector pCT5-bac2.0 $0^{11}$ by HiFi Assembly. For a control plasmid, pCT-empty was created by deletion of the sfgfp gene from pCT5-bac2.0 using the the $Q 5 \AA$ mutagenesis kit according to the manufacturers' instructions. All signal peptides (Table S4) were fused to His-EutM-SpyCatcher by site directed mutagenesis $(Q 5 \AA$ mutagenesis kit, NEB) with appropriate primers. The pCTEutMCotB plasmid for Bacillus expression was made by replacing the SpyCatcher sequence in pCT-EutMSpyC with the CotB sequence by site directed mutagenesis. To create pCT-EutMCotBEutMSpyC, the complete SacB-His-EutM-SpyCatcher expression cassette (including cumate inducible promoter) from pCT-EutMSpyC was amplified and subcloned into pCT-EutMCotB. The hag $^{\text {T209C.:SpyTag }}{ }^{588}$ expression cassette (including its native promoter) was added to pCTEutMCotB-EutMSpyC by HiFi assembly with the hag expression module amplified from the corresponding pBBRm34 plasmid (see below). Finally, to construct pCT-Purple-

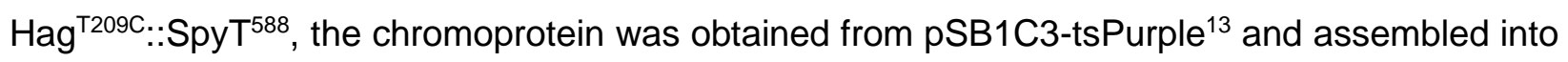
pCT-EutMCotB-EutMSpyC-hag ${ }^{T 209 C}:: S p y T^{588}$ by replacing the EutM expression modules.

\section{Plasmids for SpyTag display on B. subtilis flagella}

The flagellin gene (hag gene) was amplified from B. subtilis genomic DNA using primer pair (NPhag_fwd and NP-hag_rev) and cloned into the pRBBm34 backbone using HiFi assembly. The flagellin T209C mutation was introduced by $Q 5 \AA$-mutagenesis with primer pairs (NP-hagT209C_fwd and NP-hag-T209C_rev). A SpyTag was inserted into nine different sites between $\mathrm{D} 1 \mathrm{~b}-\mathrm{N}$ and $\mathrm{D} 1-\mathrm{C}$ domains using $\mathrm{Q} 5 \AA$-mutagenesis with primer pairs listed in Table S5.

\section{Plasmids for in-frame deletions of lytC and flhG in $B$. subtilis}

To generate the $\Delta / y t \mathrm{C}$ marker-less deletion construct, the pHBintE vector carrying a temperature sensitive origin of replication, erythromycin and ampicillin resistance was used as the backbone. The $l y t C$ upstream region was amplified from $B$. subtilis genomic DNA using primer pair 
$\mathrm{d} / y t \mathrm{C} \_u p s t r e a m \_f w d$ and $\mathrm{d} / y t \mathrm{C} \_u p s t r e a m \_r e v$ while the $l y t \mathrm{C}$ downstream region was amplified using primer pair d/ytC_downstream_fwd and d/ytC_downstream_rev (Table S5) and the deletion cassette was cloned into $\mathrm{pHBintE}$ vector using HiFi assembly. The plasmid for $\triangle$ flhG marker-less deletion was created in a similar manner: The flhG upstream region was amplified using primer pair dflhG_upstream_fwd and dflhG_upstream_rev while the flhG downstream region was amplified using primer pair dflhG_downstream_fwd and dflhG_downstream_rev (Table S5). The flhG deletion cassette was cloned into pHBintE vector using $\mathrm{HiFi}$ assembly.

\section{In-frame deletion of $/ y t C$ and $f / h G$}

The lytC deletion plasmid (temperature sensitive pHBintE-d/ytC) was transformed into $B$. subtilis using the method described by Bennallack et. al., 2014 ${ }^{9}$. After transformation, plates were incubated at a temperature permissive for plasmid replication $\left(30^{\circ} \mathrm{C}\right)$ and for selection of erythromycin resistant transformants. Colonies were then transferred into $4 \mathrm{~mL}$ LB medium without antibiotic added followed by several rounds of dilution and regrowth at a temperature nonpermissive for plasmid replication $\left(37^{\circ} \mathrm{C}\right)$. For screening of the deletion mutant appropriate dilutions of the cells were plated onto LB agar. Colonies were streaked on LB plates with and without erythromycin to identify erythromycin sensitive colonies that have successfully evicted the plasmid. Screening the erythromycin sensitive colonies for desired double homologous recombinants was performed using colony PCR with primer pair dlytC_confirmation_fwd and dlytC_confirmation_rev as shown in Table S5. Transformation of flhG deletion construct was done into $B$. subtilis $\Delta / y t C$ host using the pHBintE-dflhG plasmid. In frame deletion of flhG followed by screening was performed with same method as above for lytC but the primer pair used for screening of colonies for flhG deletion mutants was dflhG_confirmation_fwd and dflhG_confirmation_rev.

\section{Cultivation of recombinant $B$. subtilis strains}

Glycerol stocks of recombinant Bacillus strains (WT and $\Delta / y t C \Delta f / h \mathrm{G}$ deletion strain) transformed with pCT expression plasmids (pCT-empty, pCT-EutMSpyC, pCT-EutMCotB, pCT-EutMCotBEutMSpyC, pCT-EutMCotB-EutMSpyC-Hag ${ }^{\mathrm{T} 209 \mathrm{C}}: \mathrm{SSpyT}^{588}$ ) were streaked onto LB plates supplemented with tetracycline $(10 \mu \mathrm{g} / \mathrm{mL})$ and grown overnight at $30^{\circ} \mathrm{C}$. A single colony was used to inoculate $4 \mathrm{~mL}$ of selective LB. After $19 \mathrm{~h}$ of growth at $30^{\circ} \mathrm{C}, 220 \mathrm{rpm}$ strains were diluted $3 \%$ into $50 \mathrm{~mL}$ of LB or SMM supplemented with tetracycline $(10 \mu \mathrm{g} / \mathrm{mL})$ in a $250 \mathrm{~mL}$ flask. The flasks were cultured at $37^{\circ} \mathrm{C}, 180 \mathrm{rpm}$ until $\mathrm{OD}_{600}$ reached $0.4-0.7$ and protein expression was induced with $10 \mu \mathrm{M}$ cumate. The induced cultures were then grown at $20^{\circ} \mathrm{C}$ or $30^{\circ} \mathrm{C}, 100 \mathrm{rpm}$ for 48 to $96 \mathrm{~h}$ as described in this study. Final scaffold building block expression and secretion was 
done in SMM with $10 \mu \mathrm{M}$ cumate for induction and subsequent growth at $20^{\circ} \mathrm{C}, 100 \mathrm{rpm}$ for $48 \mathrm{~h}$ (referred to as "standard conditions").

For co-cultivation experiments, glycerol stocks of three $B$. subtilis $\Delta l y t C \Delta f / h G$ strains transformed with pCT-empty, pCT-EutMCotB-EutMSpyC-Hag ${ }^{\text {T209C.:SpyT }}{ }^{588}$ or pCT-Purple- Hag ${ }^{\text {T209C.:SpyT }}{ }^{588}$ were first spread onto selective LB plates, and single colonies were used to inoculate $4 \mathrm{~mL}$ of selective LB. After $19 \mathrm{~h}$ of growth at $30^{\circ} \mathrm{C}$, cultures were diluted $3 \%$ into $50 \mathrm{~mL}$ of selective SMM in a $250 \mathrm{~mL}$ flask. Individual cultures were then grown at $37^{\circ} \mathrm{C}$ until $\mathrm{OD}_{600}$ 0.4-0.7. $25 \mathrm{~mL}$ of each culture was then mixed in a new $250 \mathrm{~mL}$ flask as follows: Co-culture 1: $B$. subtilis $\Delta / y t \mathrm{C} \Delta$ flhG pCT-empty $+B$. subtilis $\Delta / y t C \Delta$ flhG pCT-Purple-Hag ${ }^{T 209 C}:: S p y T^{588}$, Co-culture 2: B. subtilis $\Delta l y t C$

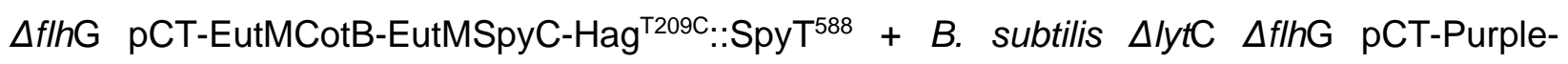
$\mathrm{Hag}^{\mathrm{T} 209 \mathrm{C}}:$ :Spy $\mathrm{T}^{588}$. Protein expression was then induced with $10 \mu \mathrm{M}$ cumate and co-cultures grown at $20^{\circ} \mathrm{C}, 100 \mathrm{rpm}$ for $48 \mathrm{~h}$.

All expression and cultivation experiments were performed with three biological replicates (i.e. three independent cultures).

\section{Analysis of scaffold building block expression and secretion by Bacillus strains}

Extracellular secretion and intracellular accumulation of scaffold building blocks by recombinant Bacillus strains was analyzed by preparing four different fractions from cultures for SDS-PAGE analysis: (i) culture supernatant, (ii) urea supernatant containing urea solubilized scaffolds that co-precipitated with cells, (iii) lysed cell pellets after solubilization of co-precipitated scaffolds, and (iv) total protein (secreted-precipitated and intracellular) from completely lysed cells with sonication followed by lysozyme treatment. The preparation of all four protein fractions is shown as a flow chart in Fig. S5a. Fractions i-iii correspond to samples A, B and C in Figs. 2, 3 and 8. Comparison of protein bands in fraction ii (i.e. absence of bands except for solubilized EutM protein) and iv indicates that urea does not break open cells Fig. S5b.

The following workflow was used for sample preparation: $5 \mathrm{~mL}$ of culture broth was centrifuged at $3,220 \times g$, at $4^{\circ} \mathrm{C}$ for 10 mins to separate culture supernatant from pelleted cells and coprecipitated scaffolds.

For SDS-PAGE analysis of soluble scaffolds in the culture supernatant (fraction i, or A in Fig. 1), proteins from $1 \mathrm{~mL}$ of supernatant were precipitated by adding $200 \mu \mathrm{L}$ of $100 \%$ Trichloroacetic acid (TCA) and $1 \mathrm{~h}$ incubation on ice. Precipitated proteins were centrifuged at $21,130 \times \mathrm{g}$ for 10 mins at $4^{\circ} \mathrm{C}$ and the supernatant was removed. The precipitate was washed with $1 \mathrm{~mL}$ of prechilled acetone and the tube was centrifuged again at $21,130 \times \mathrm{g}$ for $10 \mathrm{mins}$ at $4^{\circ} \mathrm{C}$. The acetone 
was removed and the washing step was repeated twice. The dried precipitate was resuspended in $100 \mu \mathrm{L}$ of $1 \mathrm{X}$ SDS loading buffer (2\% w/v SDS, 0.1 M DTT) (6X standard SDS loading buffer diluted with $50 \mathrm{mM}$ Tris- $\mathrm{HCl}, 4 \mathrm{M}$ urea, $\mathrm{pH}$ 7.5) for a 10-fold concentrated sample.

For SDS-PAGE analysis of secreted scaffolds that co-precipitate with cells after centrifugation of $5 \mathrm{~mL}$ cultures (fraction ii), the collected pellet was carefully resuspended in $1 \mathrm{~mL}$ of urea buffer (4 $\mathrm{M}$ urea, $50 \mathrm{mM}$ Tris- $\mathrm{HCl}$, $\mathrm{pH} 7.5)$. The resuspended solution was centrifuged at $3,220 \times \mathrm{g}$ at $4^{\circ} \mathrm{C}$ for 10 mins and the supernatant was analyzed by SDS-PAGE.

For SDS-PAGE of the remaining scaffolds in the urea washed pellet (fraction iii), cells were lysed with $200 \mu \mathrm{g} / \mathrm{mL}$ final concentration of lysozyme in $500 \mu \mathrm{L}$ of $50 \mathrm{mM}$ Tris- $\mathrm{HCl}, \mathrm{pH} 7.5$ with incubation at $37^{\circ} \mathrm{C}$ for $1 \mathrm{~h}$. The clarified supernatant after centrifugation (3,220 $\left.\mathrm{g}, 10 \mathrm{mins}\right)$ was analyzed by SDS-PAGE.

For SDS-PAGE of total protein ( $\underline{\text { fraction iv }}$ ), pelleted cells and co-precipitated scaffolds from $5 \mathrm{~mL}$ of culture was resuspended into $500 \mu \mathrm{L}$ of $50 \mathrm{mM}$ Tris- $\mathrm{HCl} \mathrm{pH} 7.5$ in an eppendorf tube. The suspension was sonicated in a coldwater bath sonicator (Bransonic 3510R-MTH, CT, USA) followed by lysis with lysozyme as above. The resulting suspension was mixed with SDS-loading buffer.

All protein samples were denatured by boiling for 10 mins at $100^{\circ} \mathrm{C}$ and $8 \mu \mathrm{L}$ of each sample was loaded onto a 15\% SDS-PAGE gel. A PowerPAC 300 power supply (Bio-Rad) was used for SDSPAGE analysis. Coomassie brilliant blue staining was then used for visualization of protein bands.

\section{De novo sequencing of secreted EutM-SpyCatcher proteins}

For de novo sequencing of secreted EutM-SpyCatcher, protein bands separated by SDS-PAGE were cut from gels and submitted to the Center for Mass Spectrometry \& Proteomics, University of Minnesota for analysis by LC-MS using a Thermo Scientific LTQ Orbitrap Velos mass spectrometer. Data were analyzed and viewed using the PEAKS Xpro Studio 10.6 software (Bioinformatics Solutions Inc., Waterloo, ON, Canada).

\section{Analysis of EutM-SpyCatcher attachment to Hag ${ }^{\mathrm{T209C}}:$ :SpyTag ${ }^{588}$ displaying flagella}

Covalent isopeptide bond formation between secreted EutM-SpyCatcher and $\mathrm{Hag}^{\mathrm{T} 209 \mathrm{C} .: \text { SpyTag }}{ }^{588}$ displayed on flagella of $B$. subtilis $\Delta l y t \mathrm{C} \Delta \mathrm{flhG}$ was analyzed by SDS-PAGE of sheared flagella isolated from co-cultures. Glycerol stocks of three B. subtilis $\Delta / y t C \Delta f / h G$

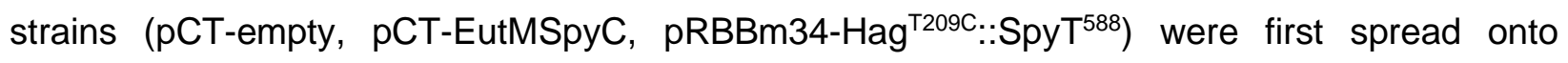


selective LB plates, and single colonies were used to inoculate $4 \mathrm{~mL}$ of selective LB liquid cultures. After $19 \mathrm{~h}$ of growth at $30^{\circ} \mathrm{C}$, cultures were diluted $3 \%$ into $50 \mathrm{~mL}$ of fresh, selective SMM and grown at $37^{\circ} \mathrm{C}$ until $\mathrm{OD}_{600}$ of $0.4-0.7$ was reached. $25 \mathrm{~mL}$ of each culture was then mixed in a new $250 \mathrm{~mL}$ flask as follows: Co-culture 1: B. subtilis $\Delta l y t \mathrm{C} \Delta$ flhG pCT-empty $+B$. subtilis $\Delta l y t \mathrm{C} \Delta \mathrm{flhG}$

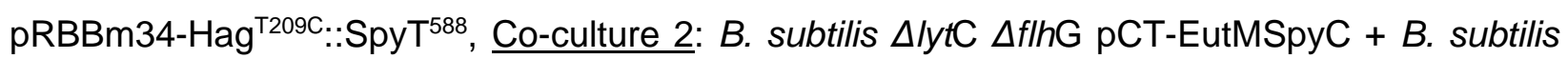
$\Delta l y t C \Delta f / h G$ pRBBm34-Hag ${ }^{T 209 C}:$. Spy ${ }^{588}$. Protein expression was induced with $10 \mu \mathrm{M}$ cumate and co-cultures grown at $20^{\circ} \mathrm{C}, 100 \mathrm{rpm}$ for $96 \mathrm{~h}$.

Cell pellets were collected from $50 \mathrm{~mL}$ cultures by centrifugation $(3,220 \times \mathrm{g}, 30 \mathrm{mins})$ and resuspended in $20 \mathrm{~mL}$ PBS $(\mathrm{pH} 7.5)$. The suspension was then sonicated at low power $(30 \%$ power, $5 \mathrm{sec}$ on and $5 \mathrm{sec}$ off, three times) (Branson 450 Digital Sonifier, CT, USA) to shear off flagella. Turbo ${ }^{T M}$ DNase (Invitrogen, Waltham, MA, USA) was then added at a $0.5 \mathrm{U} / \mathrm{mL}$ final concentration and incubated on ice for 10 mins. Cells were then removed by centrifugation at $3,220 \times g$ for 10 mins at $4^{\circ} \mathrm{C}$, followed by an ultra-centrifugation step $\left(39,800 \times g\right.$ for $2 \mathrm{~h}$ at $\left.4^{\circ} \mathrm{C}\right)$ to collect sheared flagella which were then resuspended in $100 \mu \mathrm{L} 50 \mathrm{mM}$ Tris- $\mathrm{HCl}(\mathrm{pH} 7.5)$ for SDS-PAGE analysis.

\section{Protein purification}

His-EutM, His-EutM-silica binding peptide fusion proteins and His-EutM-SpyCatcher were expressed in E. coli and purified by metal affinity chromatography as previously described ${ }^{1,2,14,15}$. His-EutM and His-EutM-silica binding peptide fusion proteins were purified using the EutM lysis buffer, while EutM-SpyCatcher was purified using the EutM-SpyCatcher buffer described in ${ }^{2}$. HistdTomato-SpyCatcher protein was expressed in E. coli and purified using the EutM-SpyCatcher protocol $^{2}$.

For silica binding assays and SEM analysis of purified His-EutM and His-EutM silica binding fusion protein, purified proteins were dialyzed against deionized water.

For TEM analysis of EutM-SpyCatcher scaffold formation in SMM, proteins expressed in E. coli or secreted by Bacillus cultures were purified with a $2 \mathrm{~mL}$ TALON metal affinity resin using a Batch/Gravity flow protein purification protocol following manufacturer's directions (TaKaRa Bio USA, Inc., see above). EutM-SpyCatcher protein from Bacillus was eluted with the same elution buffer (50 mM Tris-HCl, 250 mM NaCl, 250 mM imidazole pH 8.0) used for His-EutM-SpyCatcher as previously described ${ }^{1,2,14,15}$. EutM-SpyCatcher from E. coli was isolated from $125 \mathrm{~mL}$ cultures using the EutM-SpyCatcher protocol ${ }^{2}$ but with a TALON gravity column instead of a HisTrap ${ }^{\text {TM }}$ FF column (GE Healthcare, IL, USA). For the purification of His-EutM-SpyCatcher proteins 
secreted by $B$. subtilis $\Delta l y t C \Delta f / h G$, recombinant strains harboring $\mathrm{pCT}$-EutMSpyC were grown and induced under standard conditions described above. Cells and scaffolds from twelve $50 \mathrm{~mL}$ cultures (total volume: $600 \mathrm{~mL}$ ) were pelleted at $3,220 \times \mathrm{g}$, at $4^{\circ} \mathrm{C}$ for 10 mins and scaffolds solubilized into $30 \mathrm{~mL}$ of $50 \mathrm{mM}$ Tris- $\mathrm{HCl}, \mathrm{pH} 7.5$ containing $4 \mathrm{M}$ urea as described above. The solubilized scaffolds were then purified by TALON metal affinity chromatography as described above for E. coli. Purified protein from E. coli or Bacillus cultures were then dialyzed against SMM after elution from the TALON metal affinity resin.

For GFP labeling of scaffolds in $B$. subtilis $\Delta l y t C \Delta f / h G$ transformed with EutMCotB-EutMSpyC$\operatorname{Hag}^{\text {T209C.::Spy }}{ }^{588}$ plasmid, His-tagged eGFP and SpyTag-eGFP were expressed in $E$. coli from pET28 expression plasmids and then purified by metal affinity chromatography as described in ${ }^{2}$.

\section{Silica biomineralization and material characterization}

\section{Preparation of hydrolyzed TEOS}

Silicic acid solutions (i.e. silica) were prepared by hydrolyzing TEOS. TEOS was hydrolyzed by adding water and $1 \mathrm{M}$ hydrochloric acid at a 1:5.3:0.0013 molar ratio of TEOS:water: $\mathrm{HCl}$ and stirring vigorously. Under these conditions, hydrolysis is complete after 80 mins ${ }^{16}$.

\section{Silica binding assays on EutM scaffolds}

Silica precipitation was measured using the blue silicomolybdate method ${ }^{17,18}$. Silica was added to purified protein scaffolds, at a concentration of $1 \mathrm{mg} / \mathrm{mL}$ in deionized water, to a concentration of $100 \mathrm{mM}$ and the suspension was mixed for $2 \mathrm{~h}$ at room temperature (RT). The precipitate was then collected by centrifugation ( 3 mins at $18,800 \times g$ ), and the resulting pellet was washed 3 times with deionized water. Precipitated silica in the scaffold pellet was depolymerized by adding $1 \mathrm{M} \mathrm{NaOH}$ for 10 mins. The samples were then diluted to $1.6 \mathrm{~mL}$ and $0.15 \mathrm{~mL}$ of solution $\mathrm{A}$ (containing $20 \mathrm{~g} / \mathrm{L}$ ammonium molybdate tetrahydrate and $60 \mathrm{~mL} / \mathrm{L}$ concentrated hydrochloric acid in deionized water) was added to the sample. After 10 mins, $0.75 \mathrm{~mL}$ of solution $B$ (containing $20 \mathrm{~g} / \mathrm{L}$ oxalic acid, $6.67 \mathrm{~g} / \mathrm{L}$ 4-methylaminophenol sulphate, $4 \mathrm{~g} / \mathrm{L}$ anhydrous sodium sulfite, and $100 \mathrm{~mL} / \mathrm{L}$ concentrated sulphuric acid in deionized water) was added. The blue color was allowed to develop for $2 \mathrm{~h}$ at RT, and the absorbance was measured at $810 \mathrm{~nm}$. All samples were run in triplicate.

\section{6-well plate biomineralization experiments}

$5 \mathrm{~mL}$ of Bacillus cultures were transferred into sterile 6 -well plates. Silica was added to cultures to a concentration of $100 \mathrm{mM}$. Samples were allowed to gel at $20^{\circ} \mathrm{C}, 100 \mathrm{rpm}$ for $1 \mathrm{~h}$. The biomineralization experiments were performed with three biological replicates (i.e. three independent cultures for each experiment). 


\section{Silica block formation and mechanical testing}

Silica gel plugs were prepared in $10 \mathrm{~mL}$ syringes $(I D=15 \mathrm{~mm})$ with the tops removed and then tops were covered instead with sterilized caps to maintain sterile conditions. Silica was added to $1 \mathrm{~mL}$ cultures to a concentration of $200 \mathrm{mM}$. Samples were incubated at $25^{\circ} \mathrm{C}$ for $5 \mathrm{~h}$, and the gel plugs were pushed out of the syringe. Mechanical properties of the gel plugs were measured using an extensional DMA Rheometer (TA Instruments RSA-G2) with $15 \mathrm{~mm}$ compression disks. A frequency sweep was performed with a gap of $4.5 \mathrm{~mm}$ and an oscillation strain of $1 \%$.

Larger silica gel blocks from "purple" co-cultures of $B$. subtilis $\Delta l y t C \Delta$ flhG pCT-EutMCotB-

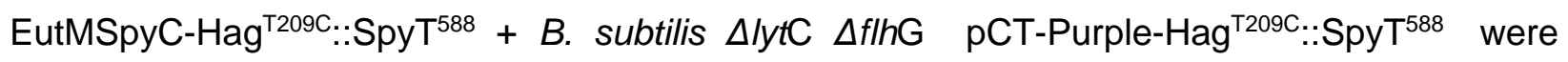
prepared in the same manner, except that $3 \mathrm{~mL}$ of culture was used and the material was cured for $24 \mathrm{~h}$.

\section{ELM regeneration}

Small piece $\left(\sim 5 \mathrm{~mm}^{3}\right)$ from silica plugs fabricated from $B$. subtilis $\Delta / y t C \Delta$ flhG pCT-EutMCotBEutMSpyC-Hag ${ }^{T 209 C .: S p y T^{588}}$ cultures (biomineralized with $200 \mathrm{mM}$ silica and cured for $24 \mathrm{~h}$ at $25^{\circ} \mathrm{C}$ ) was used to inoculate fresh cultures for ELM fabrication under standard conditions (see above). Scaffold building block expression, secretion and biomineralization in 6 -well plates by the regrown cultures was confirmed as described above.

\section{Protein analysis of silica gels}

Silica gel plugs $(15 \mathrm{~mm} \times 5 \mathrm{~mm}$ ) from $1 \mathrm{~mL}$ cultures of $B$. subtilis $\Delta / y t C \Delta$ flhG pCT-EutMCotBEutMSpyC-Hag ${ }^{\text {T209C.:SpyT }}{ }^{588}$ or pCT-empty as a control were fabricated in the same manner as described above. After $24 \mathrm{~h}$ curing at $25^{\circ} \mathrm{C}$, the entire $1 \mathrm{~mL}$ solidified silica gels were dissolved in $200 \mu \mathrm{L} 6 \mathrm{x}$ SDS loading buffer at $100^{\circ} \mathrm{C}$ for $1 \mathrm{~h}$ and $8 \mu \mathrm{L}$ of spun down sample supernatant was loaded onto $15 \%$ SDS-PAGE gel.

\section{Microscopy}

\section{Flagella light microscopy}

One $\mathrm{mL}$ of Bacillus culture was centrifuged at 2,000 x $\mathrm{g}$ for 5 mins and gently resuspended in 1 $\mathrm{mL}$ of PBS, pH 7.5. The sample was pelleted as above, and the pellet was resuspended to an $\mathrm{OD}_{600}$ of 10 . Five $\mu \mathrm{L}$ of sample was loaded onto a polysine microscope slide, covered with a coverslip, and $10 \mu \mathrm{L}$ Remel RYU flagella stain was applied to the edge of the coverslip.

Slides were examined using a Nikon Eclipse 90i microscope with a 100X 1.3 numerical aperture oil-immersion objective (University Imaging Center (UIC), University of Minnesota (UMN)). Images were captured using a Nikon D2-Fi2 color camera running on Nikon Elements software. 


\section{Spore staining}

The Schaeffer and Fulton Spore stain kit was used for spore staining with a modified protocol. Briefly, $500 \mu \mathrm{L}$ of Bacillus culture was placed into a glass test tube. One drop $(\approx 150 \mu \mathrm{L})$ of malachite green was added to the tube and immersed into boiling water for $10 \mathrm{~min}$. If samples were dehydrating before $10 \mathrm{~min}$, deionized water was added dropwise.

Following boiling, $50 \mu \mathrm{L}$ of sample was spread by pipette onto a plain, precleaned glass slide. The slide was allowed to air dry and then heat fixed with flame. The slide was decolorized with distilled water, counterstained with safranin for $\approx 20$ seconds and again decolorized.

Slides were examined using a Nikon Eclipse 90i microscope with a 100X 1.3 numerical aperture oil-immersion objective (UIC UMN). Images were captured using a Nikon D2-Fi2 color camera running on Nikon Elements software.

\section{SEM imaging}

EutM scaffolds ( $1 \mathrm{mg} / \mathrm{mL}$ purified proteins in water) were attached to silicon wafers by incubating the protein suspensions on a silicon wafer for $1 \mathrm{~h}$. In samples with silica, the wafers with attached protein were incubated in $100 \mathrm{mM}$ silica for $2 \mathrm{~h}$. Samples were washed with water, fixed with Trump's Fixative for two min, and washed with water again. Samples were washed with increasing ethanol concentrations of $25 \%, 50 \%, 75 \%$, and $100 \%$, and then supercritically dried using a Tousimis Critical-Point Dryer (Model 780A). Samples were sputter-coated with $8 \mathrm{~nm}$ of iridium and viewed with a Hitachi SU8230 field emission gun scanning electron microscope (University of Minnesota Characterization Facility).

\section{T209C flagella staining}

Glycerol cultures were inoculated into $4 \mathrm{~mL}$ of selective LB medium for plasmid maintenance and grown overnight at $30^{\circ} \mathrm{C}$ and $220 \mathrm{rpm}$. One $\mathrm{mL}$ of culture was centrifuged at 2,000 $\mathrm{g}$ for 10 mins. The supernatant was discarded and pelleted cells were gently resuspended in $1 \mathrm{~mL}$ of $\mathrm{PBS}, \mathrm{pH}$ 7.5. The samples were pelleted as above and pellets resuspended with $50 \mu \mathrm{L}$ PBS, $\mathrm{pH} 7.2$ with $5 \mu \mathrm{g} / \mathrm{mL}$ Alexa Fluor ${ }^{\mathrm{TM}} 488 \mathrm{C}_{5}$ maleimide (to stain cysteines in flagella with the $\mathrm{Hag}^{\mathrm{T} 209 \mathrm{C}}$ subunit) and incubated in the dark for five mins. $450 \mu \mathrm{L}$ PBS was added to the samples prior to centrifugation as above. The pellet was resuspended in $50 \mu \mathrm{L}$ PBS with $5 \mu \mathrm{g} / \mathrm{mL}$ SynaptoRed ${ }^{\mathrm{TM}}$ C2 (FM4-64) and incubated for five mins in the dark. Samples were pelleted as above and then resuspended with $1 \mathrm{~mL}$ of PBS. After another centrifugation step, the pellets were resuspended to an $\mathrm{OD}_{600}$ of 10 . Three $\mu \mathrm{L}$ of sample were mixed with an equal volume of anti-fade fluorescent mounting medium FluoroShield and applied to a polysine microscope slide. 
Slides were examined using a Nikon Eclipse 90i microscope with a 100X 1.3 numerical aperture oil-immersion objective (UIC UMN). Illumination was obtained using a Lumencor Sola Light Engine. SynaptoRed ${ }^{\mathrm{TM}} \mathrm{C} 2$ was visualized using a dsRed fluorescence cube (excitation 530-560 $\mathrm{nm}$, emission 590-650 nm) with a 3 second exposure time. Alexa Fluor ${ }^{\mathrm{TM}} 488 \mathrm{C}_{5}$ maleimide was visualized using a GFP fluorescence cube (excitation 450-490 nm, emission 500-550 nm) with a 5.6 second exposure time. Images were captured using a Hamamatsu Orca Flash 4.0 v2 CMOS monochrome camera in black and white using Nikon Elements software.

\section{In situ labeling of SpyTagged flagella with His-tdTomato-SpyCatcher}

Glycerol cultures were inoculated into $4 \mathrm{~mL}$ of selective LB medium containing $0.15 \mathrm{mg}$ of purified His-tdTomato-SpyCatcher protein and grown overnight at $30^{\circ} \mathrm{C}$ and $220 \mathrm{rpm}$. One $\mathrm{mL}$ of culture was centrifuged at 2,000 $\times \mathrm{g}$ for 10 mins and gently resuspended in one $\mathrm{mL}$ of PBS, $\mathrm{pH}$ 7.5. The samples were pelleted as above. The pellets were resuspended with $50 \mu \mathrm{L}$ PBS pH 7.2 with 5 $\mu \mathrm{g} / \mathrm{mL}$ Alexa Fluor ${ }^{\mathrm{TM}} 488 \mathrm{C}_{5}$ maleimide and incubated in the dark for five mins. $450 \mu \mathrm{L}$ PBS was added to the samples prior to centrifugation as above. Cellular nucleic acid was stained by resuspending the pellets in $50 \mu \mathrm{L}$ Tris- $\mathrm{HCl}, \mathrm{pH} 8.0$ with $10 \mu \mathrm{M}$ Syto16 and incubated in the dark for 10 mins. $450 \mu \mathrm{L}$ Tris- $\mathrm{HCl}, \mathrm{pH} 8.0$ was added and samples were centrifuged as above. Unused dye was removed by resuspension with $1 \mathrm{~mL}$ PBS and samples were centrifuged again. After resuspension to an $\mathrm{OD}_{600}$ of $10,3 \mu \mathrm{L}$ of sample was mixed with an equal volume of FluoroShield and loaded onto a polysine microscope slide.

Slides were examined using a Nikon Eclipse 90i microscope with a 100X 1.3 numerical aperture oil-immersion objective (UIC UMN). Illumination was obtained using a Lumencor Sola Light Engine. tdTomato was visualized using a dsRed fluorescence cube (excitation 530-560 nm, emission 590-650 nm) with a 3 second exposure time. Alexa Fluor ${ }^{\mathrm{TM}} 488 \mathrm{C}_{5}$ maleimide and Syto ${ }^{\mathrm{TM}} 16$ were visualized using a GFP fluorescence cube (excitation 450-490 nm, emission 500$550 \mathrm{~nm}$ ) with a 0.3-5.6 second exposure time. Images were captured using a Hamamatsu Orca Flash 4.0 v2 CMOS monochrome camera in black and white using Nikon Elements software.

\section{TEM of purified scaffolds from E. coli and Bacillus}

Purified His-EutM-SpyCatcher protein scaffolds were diluted to $1 \mathrm{mg} / \mathrm{mL}$. Ten $\mu \mathrm{L}$ was placed onto 200 mesh copper grids with $10 \mathrm{~nm}$ formvar and $1 \mathrm{~nm}$ carbon for 3 mins. The fluid was removed and $10 \mu \mathrm{L}$ of Trump's fixative (4\% formalin, $1 \%$ glutaraldehyde, $0.1 \mathrm{M}$ sodium cacodylate) was applied for 5 mins to fix the proteins. The fluid was removed and the grids were rinsed with $10 \mu \mathrm{L}$ of deionized water three times. Negative staining was completed using $1 \%$ aqueous uranyl acetate which was applied and removed immediately to prevent over-staining. Grids were 
examined using a JEOL-JEM-1400Plus transmission electron microscope with a LaB6 tungsten filament at $60 \mathrm{kV}$ (UIC UMN). Images were acquired using an Advanced Microscopy Techniques XR16 camera using AMT capture Engine software version 7.0.0.187.

\section{In situ labeling of secreted EutM-SpyCatcher scaffolds with His-SpyTag-eGFP}

B. subtilis $\Delta / y t C \Delta$ flhG harboring pCT-EutMCotB-EutMSpyC-Hag ${ }^{T 209 C}$ ::Spy ${ }^{588}$ was grown under standard conditions with (induced) and without (uninduced) cumate added for induction of protein expression. $500 \mu \mathrm{L}$ of culture was mixed with $0.25 \mathrm{mg}$ His-eGFP or His-SpyTag-eGFP (an estimated 2:1 ratio of SpyCatcher : eGFP established in ${ }^{7}$ ) and mixed at RT for 30 mins. Ten $\mu \mathrm{L}$ was applied to a glass slide and covered with a slip. Slides were examined using a Nikon Eclipse 90i microscope with a 40X 0.75 numerical aperture objective (UIC UMN). Illumination was obtained using a Lumencor Sola Light Engine. His-eGFP and His-SpyTag-eGFP were visualized using a GFP fluorescence cube (excitation 450-490 nm, emission 500-550 nm) with a 0.032-0.2 second exposure time. DIC images were captured with a 0.037-0.039 second exposure. Images were captured using a Hamamatsu Orca Flash 4.0 v2 CMOS monochrome camera in black and white using Nikon Elements software.

\section{Silica material thin sections and TEM}

B. subtilis $\Delta / y t C \Delta$ flhG harboring pCT-EutMCotB-EutMSpyC-Hag ${ }^{\mathrm{T} 209 \mathrm{C} .: \text { Spy }^{588}}{ }^{5 a s}$ grown under standard conditions with (induced) and without (uninduced) cumate added for induction of protein expression. Cultures with cell densities of $\mathrm{OD}_{600} \sim 3.5$ (induced) or $\sim 6$ (uninduced) were either directly silica biomineralized or were concentrated prior to silica gel formation. For concentration, cultures were centrifuged at 3,220 $\mathrm{x} g$ for 10 mins and the supernatant was removed. Pellets were resuspended to $\mathrm{OD}_{600} 10$ or 20 using the media removed from the pellet. Silica gel plugs were prepared as described above for mechanical testing using $200 \mathrm{mM}$ silica. Gels were pushed out of the syringes and $2 \mathrm{~mm}^{3}$ gel portions were cut from the center of both induced and uninduced samples. The gel pieces were fixed at $4^{\circ} \mathrm{C}$ and $10 \mathrm{rpm}$ overnight in $100 \mathrm{mM}$ sodium cacodylate, $2 \%$ glutaraldehyde, and $2 \%$ paraformaldehyde. The gel pieces were then rinsed with $100 \mathrm{mM}$ sodium cacodylate three times for 30 mins each at $4^{\circ} \mathrm{C}$ and $10 \mathrm{rpm}$. An overnight post-fixation using $4 \%$ osmium tetraoxide in $100 \mathrm{mM}$ sodium cacodylate was completed at $4^{\circ} \mathrm{C}$ and $10 \mathrm{rpm}$. The samples were extensively rinsed with water and subjected to an ethanol dehydration series (25\%, 50\%, 75\%, 95\%, 100\%). Gel samples were trimmed into approximately $1 \mathrm{~mm}^{3}$ pieces, infiltrated with EMbed 812 resin (1:2 resin:ethanol, 1:1, 2:1, 100\% resin without hardener, 100\% resin with benzyl dimethyl amine hardener repeated once (2x total); all times 8 h-overnight), and polymerized in a $60^{\circ} \mathrm{C}$ oven for $48 \mathrm{~h}$. Sections were cut using a diamond knife on a Leica Ultracut UCT microtome at a thickness of 70-100 microns and collected on 200-mesh formvar/carbon- 
coated copper grids. Sections were stained with 3\% aqueous uranyl acetate for 20 mins, rinsed in ultrapure water ( $5 \mathrm{sec}, 5 \mathrm{x}$ ), stained with Sato's triple-lead $\operatorname{stain}^{19}$ for 3 mins, and rinsed in ultrapure water (5 sec, 5x). Samples were examined using a JEOL-JEM-1400Plus transmission electron microscope with a LaB6 tungsten filament at $60 \mathrm{kV}$ (UIC UMN). Images were acquired using an Advanced Microscopy Techniques XR16 camera using AMT capture Engine software version 7.0.0.187.

\section{Image Analysis}

Images were cropped, scale bars added, and channels were colorized and merged using Fiji v. $1.53^{20}$. Fig. $S 9$ image edits for part A include $B$. subtilis $\Delta / y t C \Delta f / h G$ strain SpyTag 555 in which the red and green channels were multiplied by 1.25 before merging. Fig. $\mathbf{S 1 2}$ image edits for part A include the $\mathrm{Hag}^{\mathrm{T} 209 \mathrm{C}}$ control in which the red channel was multiplied by 1.5 before merging with the green channel. In addition, for $\mathrm{Hag}^{\mathrm{T} 209 \mathrm{C}}:$ :SpyTag ${ }^{588}$ the red channel was multiplied by 1.25 before merging. Fig. S12 image edits in part B include the $\mathrm{Hag}^{\mathrm{T} 209 \mathrm{C}}$ control in which the red

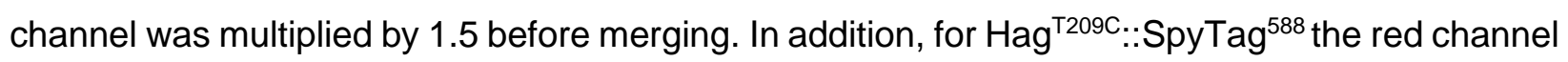
was multiplied by 1.25 before merging.

Flagella RYU staining images were converted to grayscale and had the contrast and brightness increased by 10 using GNU Image Manipulation Program (GIMP) v. 2.8.18 (https://www.gimp.org/). 


\section{SUPPORTING FIGURES}
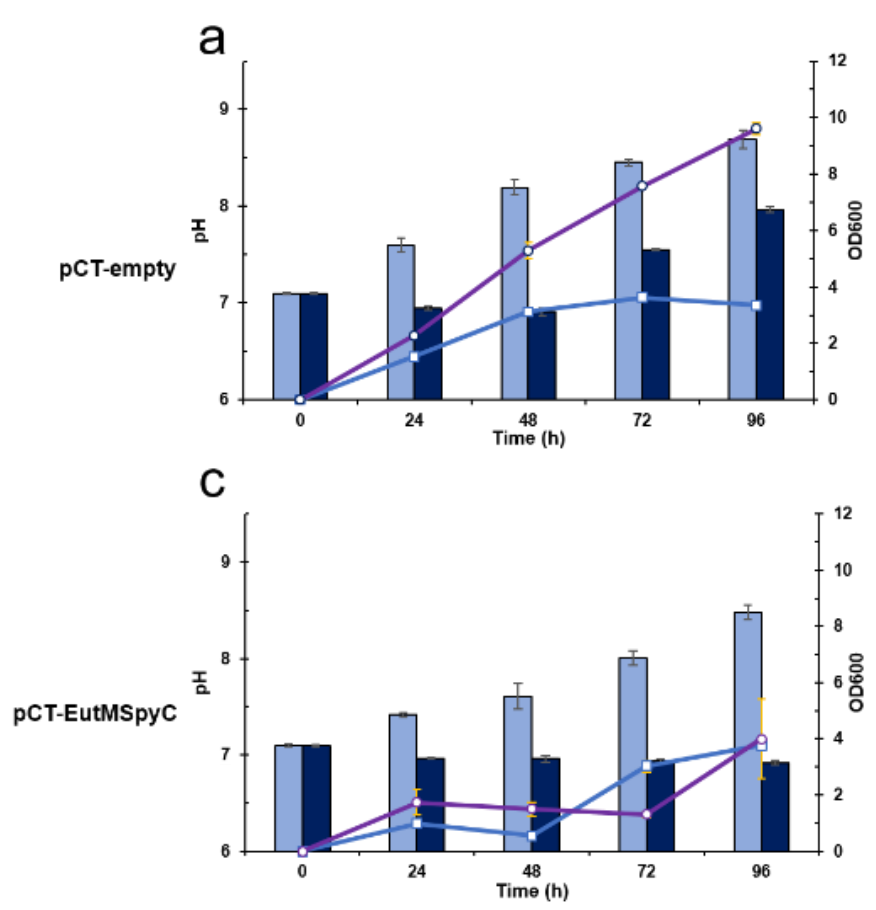

b

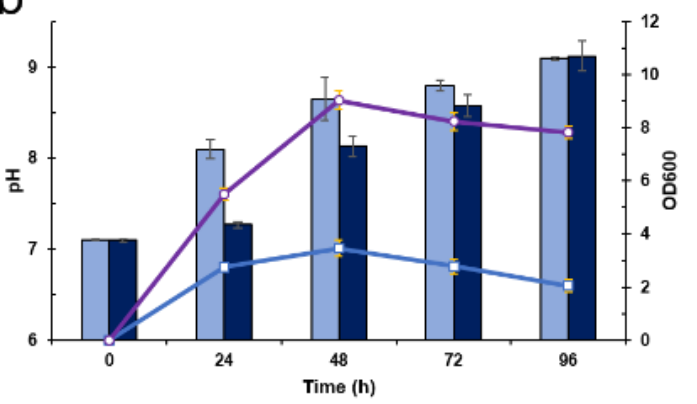

d

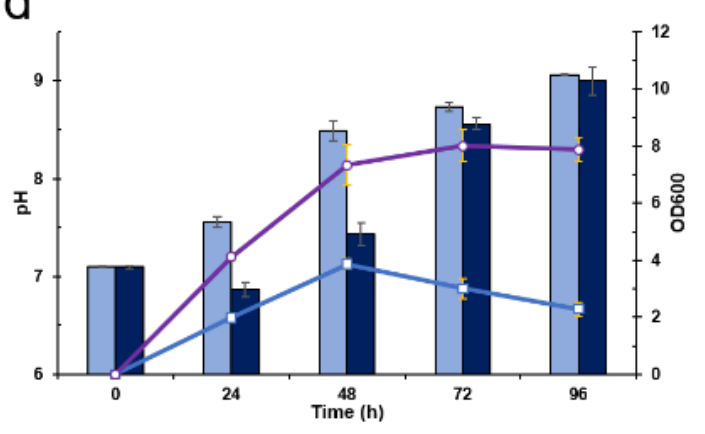

$$
\begin{array}{ll}
\mathrm{pH} & \mathrm{OD}_{600} \\
\square & -\mathrm{-} \\
\square & \text { LB } \\
\square & \text { sMM }
\end{array}
$$

Fig. S1: Establishing growth conditions suitable for silica biocomposite formation with $B$. subtilis pCT-empty and pCT-EutMSpyC.

Growth of $B$. subtilis WT strain transformed with the cumate inducible, pCT-empty expression plasmid was compared in two media (LB and SMM, $100 \mathrm{rpm}$ ) at (a) $20^{\circ} \mathrm{C}$ and (b) $30^{\circ} \mathrm{C}$. Similarly, growth of B. subtilis WT transformed with PCT-EutMSpyC was compared in LB and SMM at (c) $20^{\circ} \mathrm{C}$ and $(\mathbf{d}) 30^{\circ} \mathrm{C} . \mathrm{OD}_{600}$ and $\mathrm{pH}$ of cultures were followed for $96 \mathrm{~h}$. Cultures were induced with $10 \mu \mathrm{M}$ cumate once they reached an $\mathrm{OD}_{600}$ of $0.4-0.7$. B. subtilis WT strain transformed with pCTEutMSpyC (c) show changes in growth rates at $20^{\circ} \mathrm{C}$ suggesting diauxic growth and/or an increased metabolic burden caused by recombinant protein expression. Error bars represent standard deviations calculated for values from three biological replicate cultures. 
a

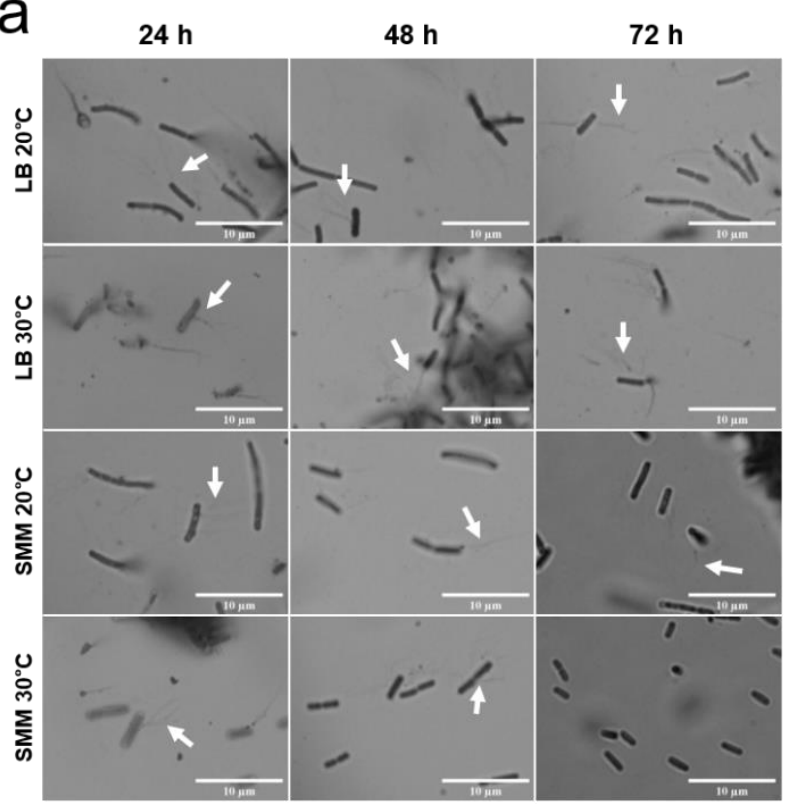

b $24 \mathrm{~h}$

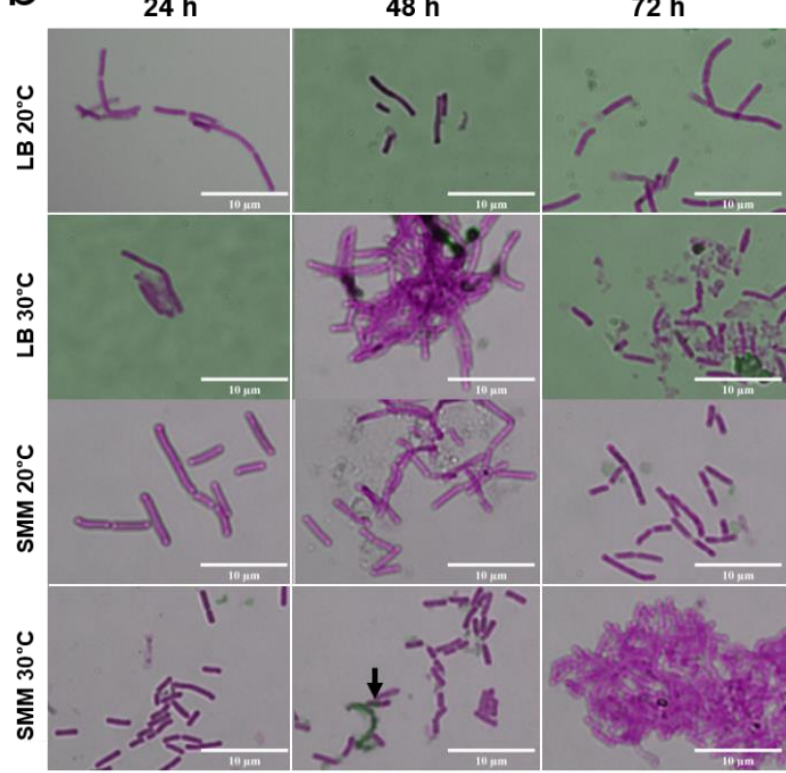

Fig. S2: Analysis of spore formation and flagella phenotypes of B. subtilis pCT-empty under different growth conditions.

Spore formation and flagellation of $B$. subtilis WT pCT-empty was analyzed under different growth conditions. At $24 \mathrm{~h}$ time intervals, samples were removed from SMM and LB cultures grown at $20^{\circ} \mathrm{C}$ or $30^{\circ} \mathrm{C}$ (shown in Figure S1) for staining and imaging by light microscopy. Cells were stained with RYU to observe flagella (a) or malachite green and safranin red to visualize spores (b). Flagella were observed through $72 \mathrm{~h}$ of growth, with the most flagella present at $48 \mathrm{~h}$ of growth or less under all conditions. Only one, unreleased spore was observed at $48 \mathrm{~h}$ of growth in $\mathrm{SMM}$ at $30^{\circ} \mathrm{C}$, corresponding to reaching stationary phase. 

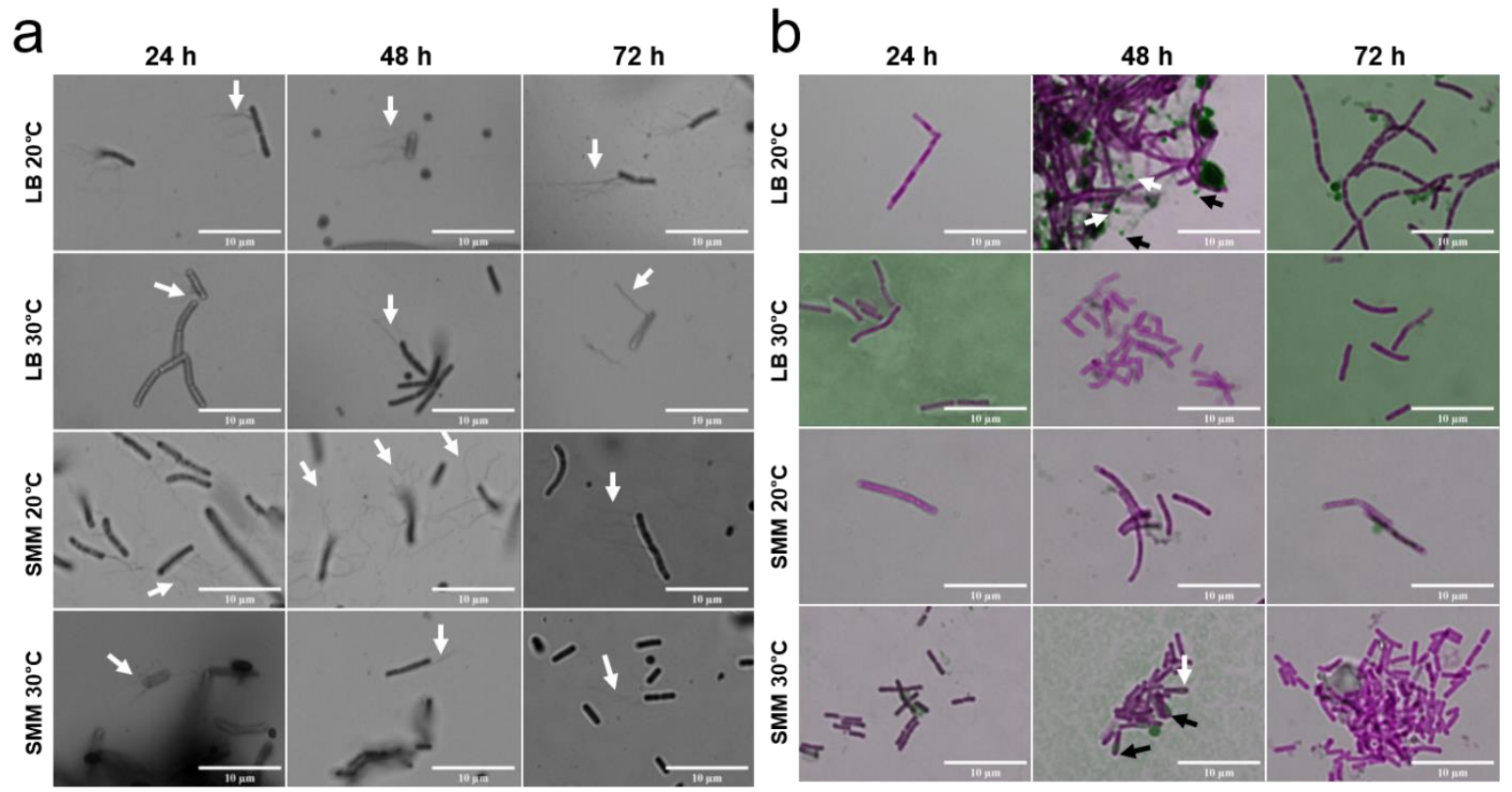

Fig. S3: Analysis of spore formation and flagella phenotypes of $B$. subtilis pCT-EutMSpyC.

Spore formation and flagellation of $B$. subtilis WT transformed with pCT-EutMSpyC was analyzed under different growth conditions. At $24 \mathrm{~h}$ time intervals, samples were removed from SMM and LB cultures grown at $20^{\circ} \mathrm{C}$ or $30^{\circ} \mathrm{C}$ (shown in Fig. S1) for staining and imaging by light microscopy. Cells were stained with RYU to observe flagella (a) or malachite green and safranin red to visualize spores (b). Flagella were observed through $72 \mathrm{~h}$ of growth, with the most flagella present at $48 \mathrm{~h}$ of growth or less under all conditions. Sporulation was observed after $48 \mathrm{~h}$ in LB medium at $20^{\circ} \mathrm{C}$, correlating with a corresponding decrease in $\mathrm{OD}_{600}$ due to cell lysis (see Fig. S1) while the few spores visible at $48 \mathrm{~h}$ in $\mathrm{SMM}$ at $30^{\circ} \mathrm{C}$ did not impact OD. 
a

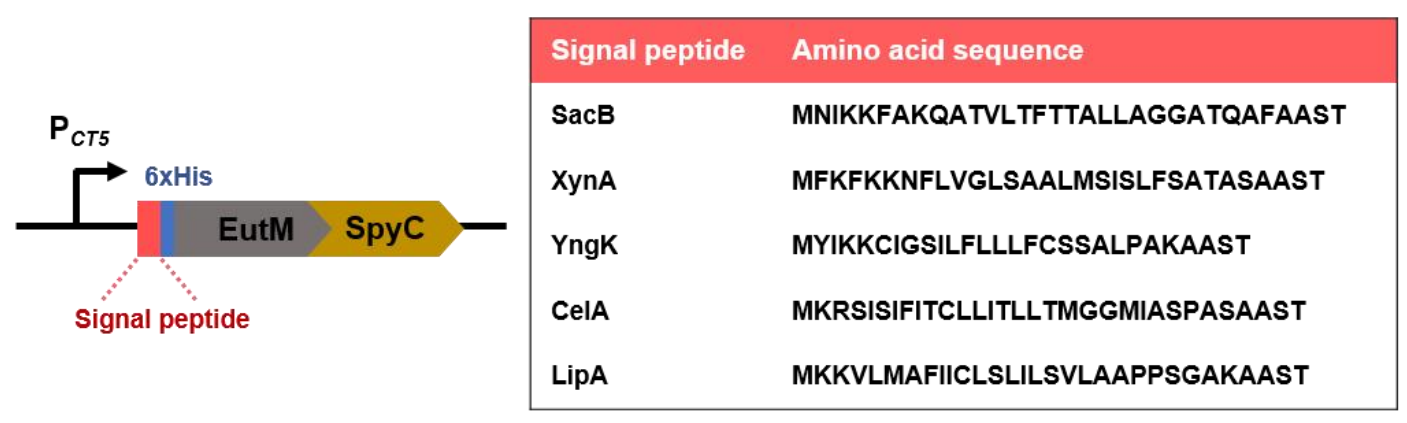

b

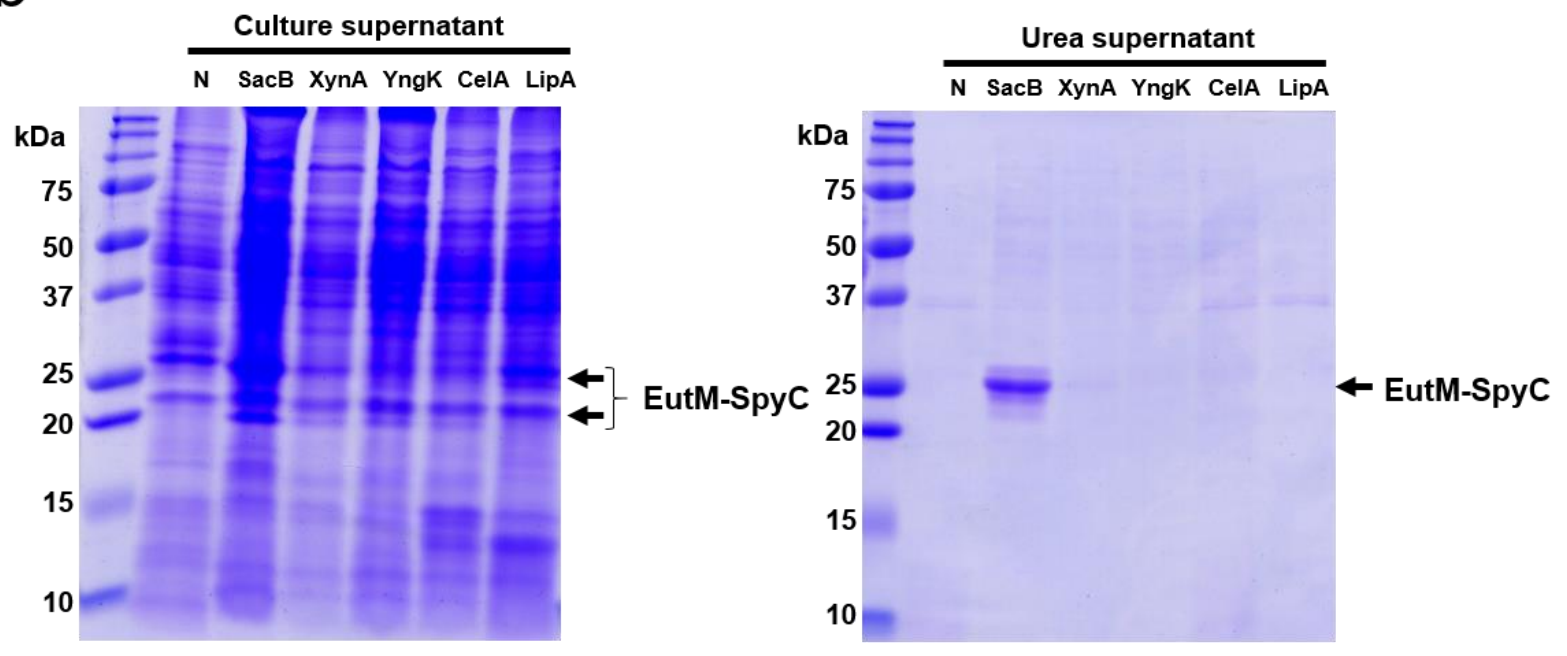

Fig. S4: Testing of additional secretion signal sequence for scaffold building block secretion by $B$. subtilis.

a Scaffold building block secretion levels by $B$. subtilis WT was compared with five commonly used secretion signal peptides placed upstream of the His-EutM-SpyCatcher region.

b Cultures expressing the EutM-SpyCatcher proteins were grown for $48 \mathrm{~h}$ in $S M M$ at $20^{\circ} \mathrm{C}$ under induction condition to analyze protein secretion by SDS-PAGE. Culture supernatants were collected and concentrated 25 -fold by TCA precipitation prior to analysis (left panel). Cell pellets were then treated with $4 \mathrm{M}$ urea (see Fig. 2) to solubilize co-precipitated EutM-SpyCatcher scaffolds for analysis (right panel). B. subtilis transformed with a PCT-empty vector was included as negative control $(\mathrm{N})$. Only the SacB signal sequence facilitated secretion of EutM-SpyCatcher in the supernatant and as co-precipitant in the cell pellet. 
a
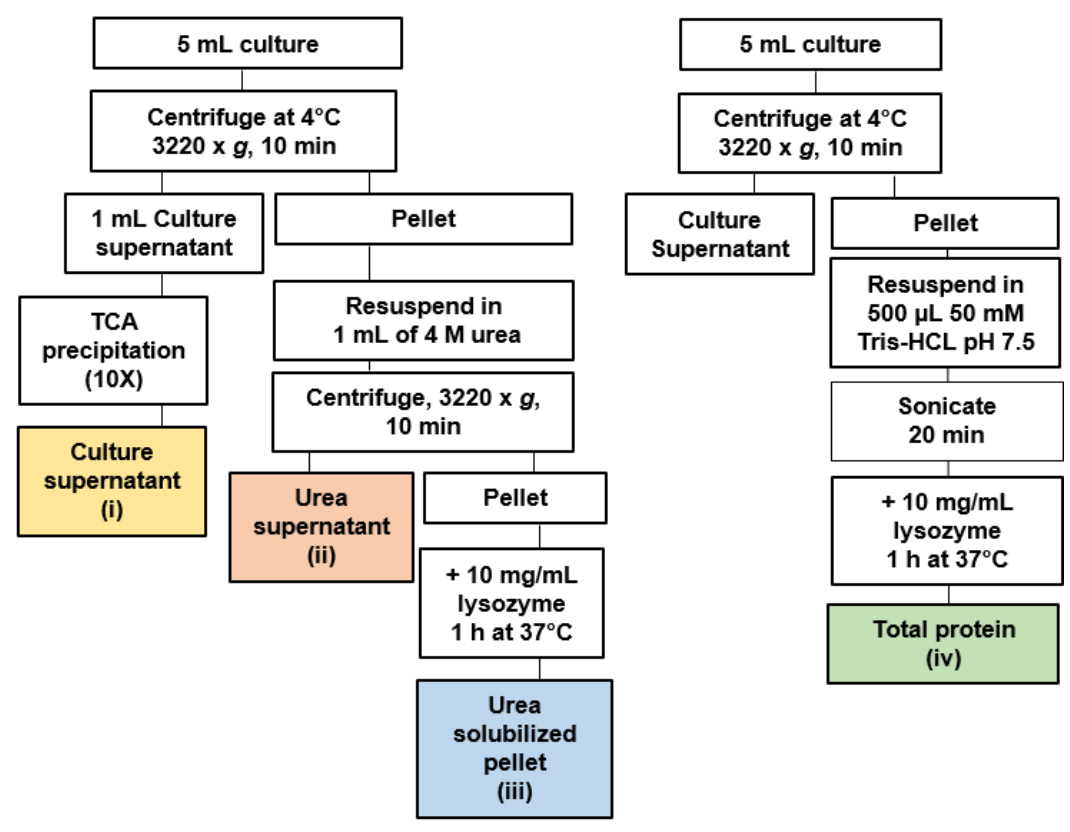

b

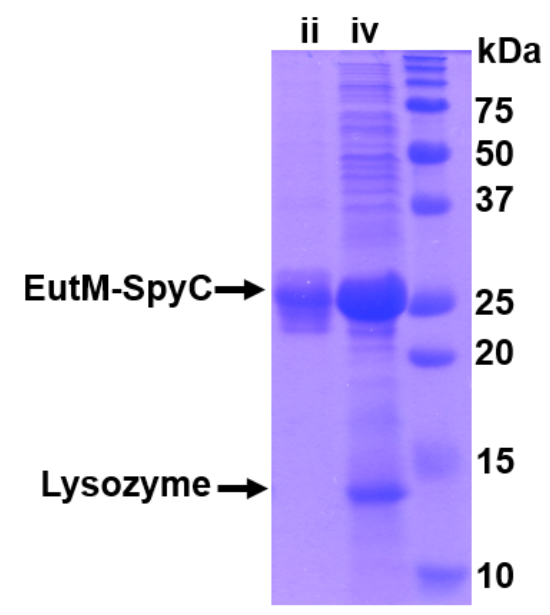

Fig. S5: Experimental workflow used for the analysis of EutM scaffold building block secretion and expression by engineered $B$. subtilis.

Different fractions of proteins were prepared by B. subtilis WT pCT-EutMSpyC after $48 \mathrm{~h}$ of induction in $\mathrm{SMM}$ at $20^{\circ} \mathrm{C}$.

a Workflow for the preparation of the following fractions containing EutM-SpyCatcher protein: (i) culture supernatant, (ii) urea supernatant, (iii) urea solubilized pellet and (iv) total protein. Fractions i-iii correspond to $A, B$ and $C$ in Fig. 2, 3 \& 8. Fraction iv represents total protein (intracellular and precipitated scaffolds) in sedimented pellet after complete lysis of cells.

b Comparison of proteins solubilized by urea treatment from pelleted cells and scaffolds (fraction ii) and total protein released from broken cells and sedimented scaffolds (fraction iv). Urea treatment does solubilize EutM scaffolds but does not break cells. 
a

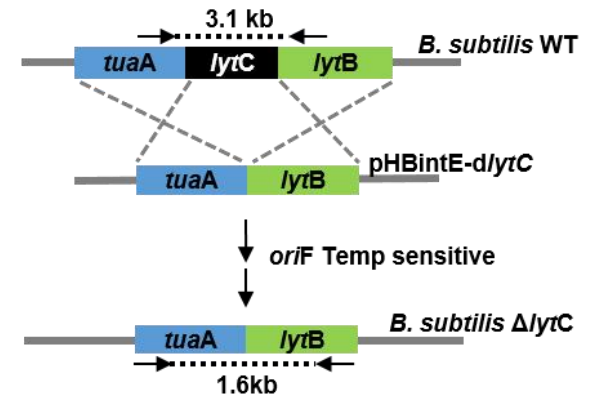

b
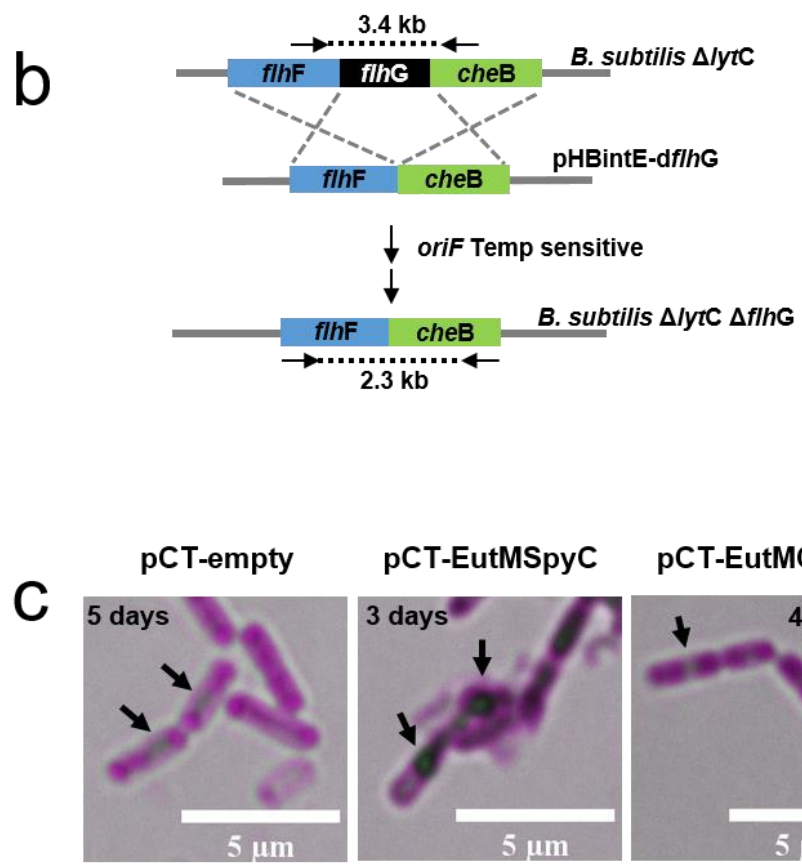

d

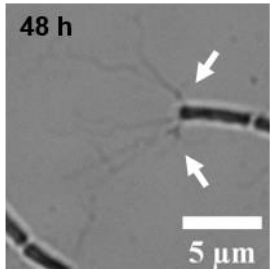

pCT-EutMSpyC
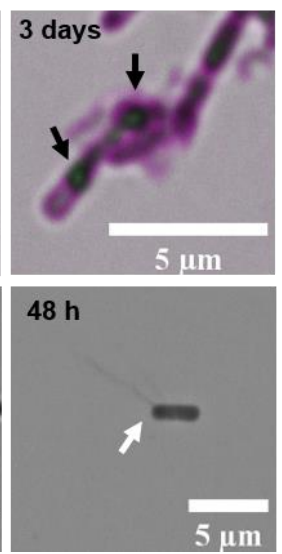

pCT-EutMCotB
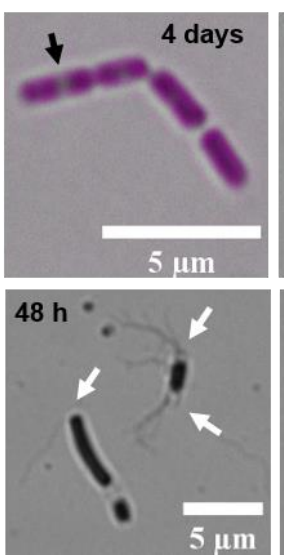

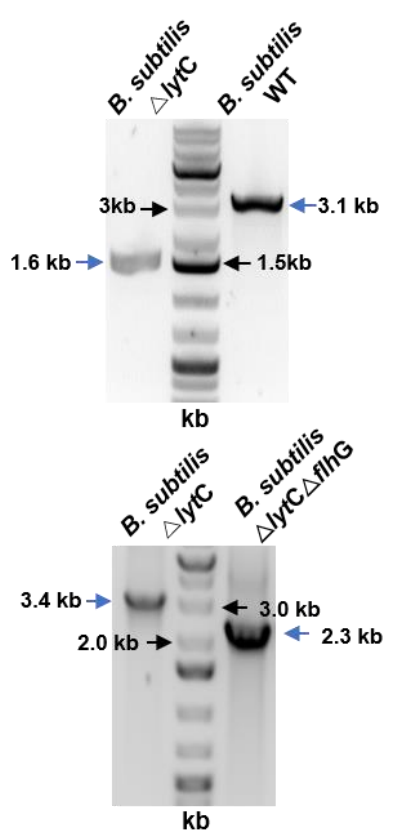

pCT-EutMCotB-

pCT-EutMCotB- EutMSpyCEutMSpyC

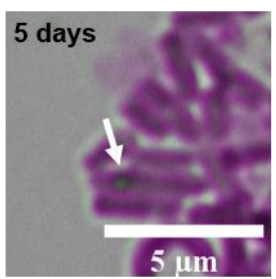

$\operatorname{Hag}^{\top 209 c:: S p y T^{588}}$
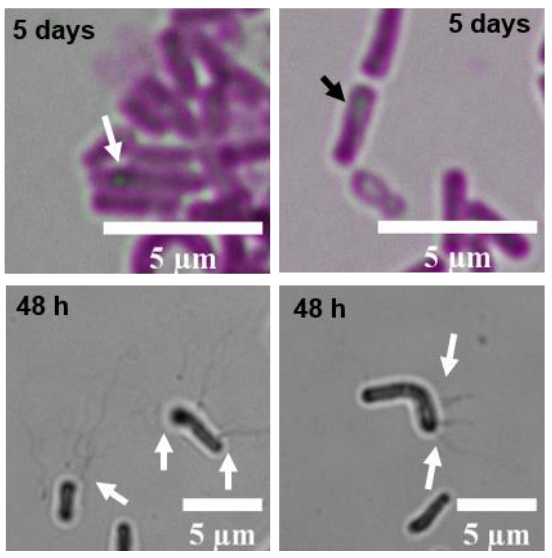

Fig. S6: Construction and phenotypical characterization of the double deletion strain $B$. subtilis $\Delta / y t C \Delta$ flhG as ELM fabrication chassis.

Deletions of genes encoding the autolysin LytC (a) and flagellar basal body protein FlhG (b) were made in B. subtilis 168 (WT strain) by consecutive rounds of homologous recombination with a temperature sensitive plasmid. In frame deletions were confirmed by PCR, yielding amplification products with the expected sizes. Endospore formation (c) and polar flagella clusters (d) were then confirmed for the double deletion strain transformed with different expression plasmids used in this work. B. subtilis $\Delta / y t C \Delta f / h G$ strains were cultured in $S M M$ at $20^{\circ} \mathrm{C}$ under induction condition. Spores were visualized with malachite green and safranin red at days after induction as indicated (when endospores formed). Red was replaced with magenta using FIJI software (c). Flagella of the same cultures RYU stained and imaged after $48 \mathrm{~h}$ of induction. Images were converted to grayscale using GIMP software $(\mathbf{d})$. 


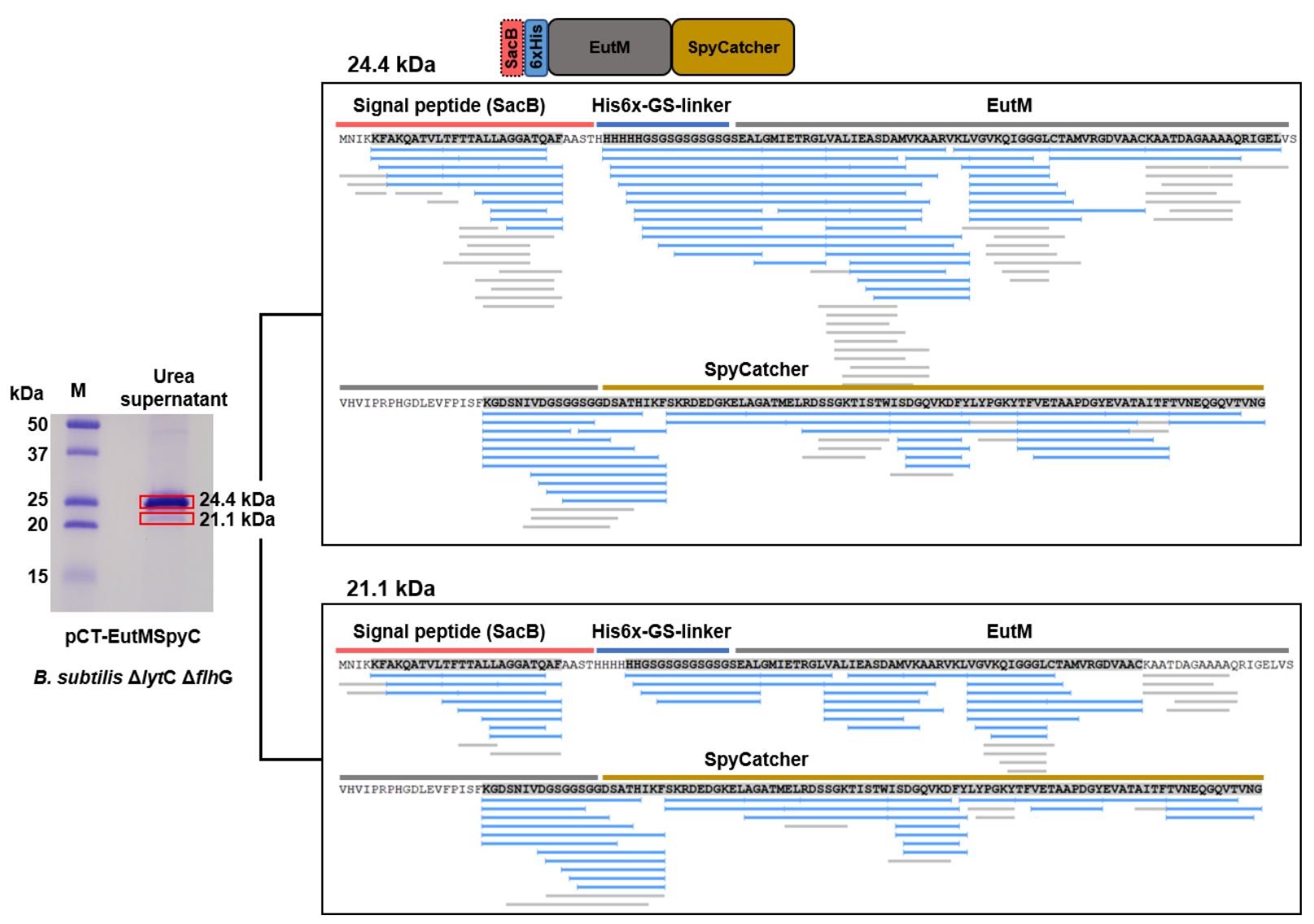

Fig. S7: Analysis of EutM-SpyCatcher secreted by B. subtilis $\Delta / y t C \Delta$ flhG.

SDS-PAGE analysis and LC-MS de novo sequencing of EutM-SpyCatcher proteins produced by B. subtilis $\Delta / y t C \Delta$ flhG pCT-EutMSpyC after $48 \mathrm{~h}$ of induction in SMM at $20^{\circ} \mathrm{C}$. Two bands $(21.1$ and $24.4 \mathrm{kDa}$ ) are visible in the urea supernatant (see Fig. 3d). Boxed bands were cut and subjected to de novo sequencing by LC-MS. Coverages of sequenced peptides were visualized by PEAKS Studio Viewer shown to the right. Under these expression conditions, both protein bands have an uncleaved SacB signal peptide. A segment after the signal peptide cleavage site (AFA) was not sequenced in addition to other sequence regions. To confirm that the LC-MS sequencing results were not due to plasmid mutations that occurred during cultivation in the signal peptidase cleavage site regions, plasmids isolated from 10 colonies obtained from the $B$. subtilis $\Delta / y t C \Delta f / h G$ pCT-EutMSpyC were Sanger sequenced to cover the signal peptide and the EutMSpyCatcher region. No mutations were found. Peptide fragments sequenced corresponding to the $\mathrm{C}$-terminus of both protein bands indicate that the minor, smaller $21.1 \mathrm{kDa}$ band corresponds to an isoform that migrates faster in the SDS-PAGE gel. 

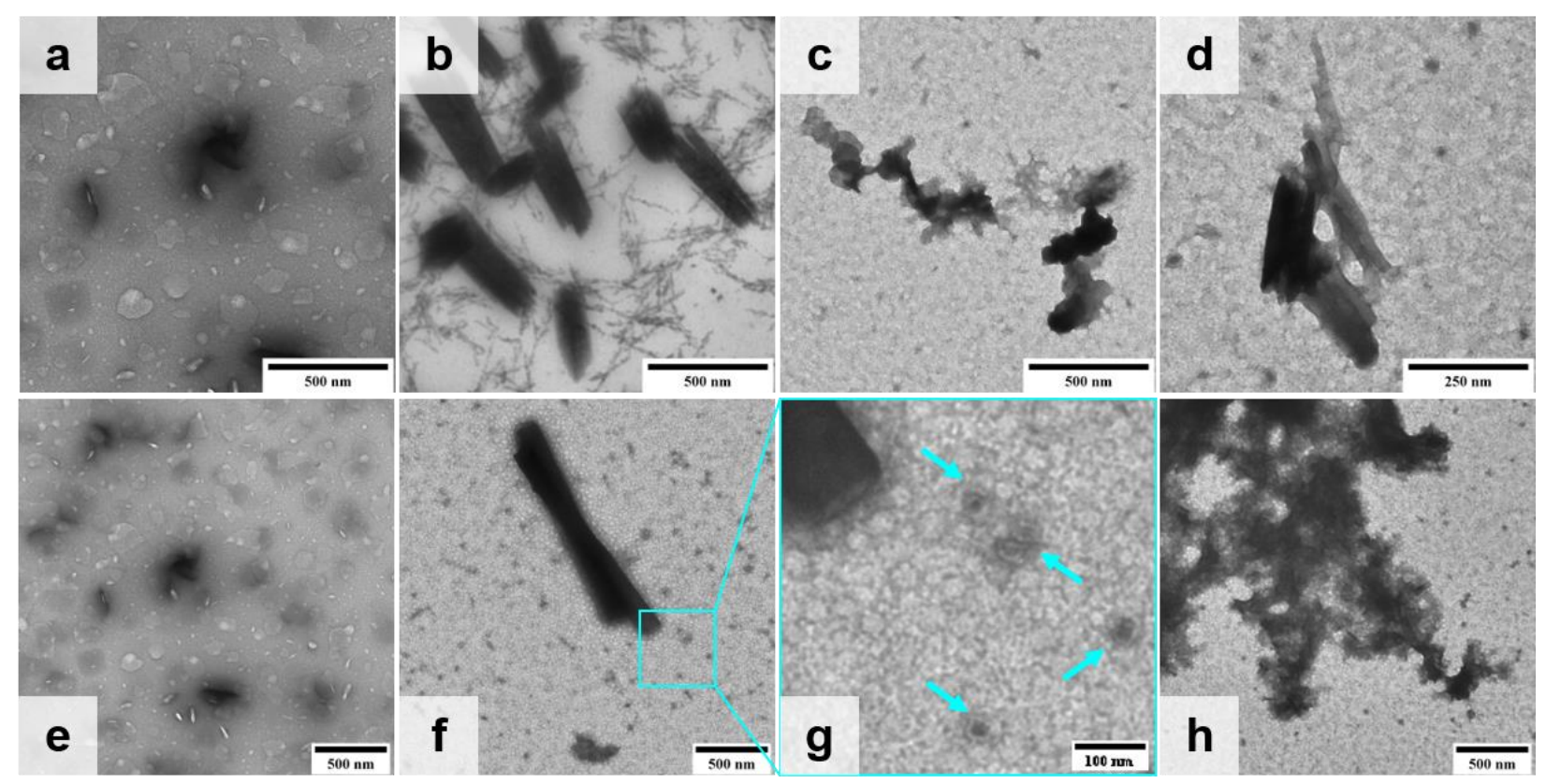

Fig. S8: Comparison of scaffold formation by EutM-SpyCatcher purified from recombinant $E$. coli and $B$. subtilis $\Delta / y t C \Delta f / h G$ cultures.

His-tagged EutM-SpyCatcher from E. coli and urea solubilized scaffolds of $B$. subtilis $\Delta / y t C \Delta$ flhG pCT-EutMSpyC cultures were purified by metal affinity chromatography as described in the methods. The eluted proteins were dialyzed into SMM (a, e media blank used for $E$. coli and $B$. subtilis protein dialysis, respectively) to remove imidazole and urea for analysis of scaffold formation in this medium. The SMM dialyzed EutM-SpyCatcher proteins ( $E$. coli $1.8 \mathrm{mg} / \mathrm{mL}, B$. subtilis $2.3 \mathrm{mg} / \mathrm{mL}$ ) were diluted to $1 \mathrm{mg} / \mathrm{mL}$ with sterile $S M M$ and structures were visualized by TEM. a-c are magnified $10,000 \times \mathbf{~} \mathbf{d}$ is $20,000 \times \mathbf{e}, \mathbf{e}, \mathbf{f}, \mathbf{h}$ are $6,000 \times \mathbf{~} \mathbf{g}$ is enlarged $5 \times$ from $\mathbf{f}$.

b Rolled up tubes were visible in $E$. coli protein samples and have been observed previously for EutM scaffolds ${ }^{1,2}$. c, d, f-h Rolled up tubes, sheets, coral- and small spherical structures (that stain as donut-like structures) were visible in Bacillus samples and have also been previously observed for EutM scaffolds ${ }^{1,2,4-6}$. The enlarged spherical structures from $\mathbf{f}$ are also present in $\mathbf{c}$, $\mathbf{d}$, and $\mathbf{h}$. 


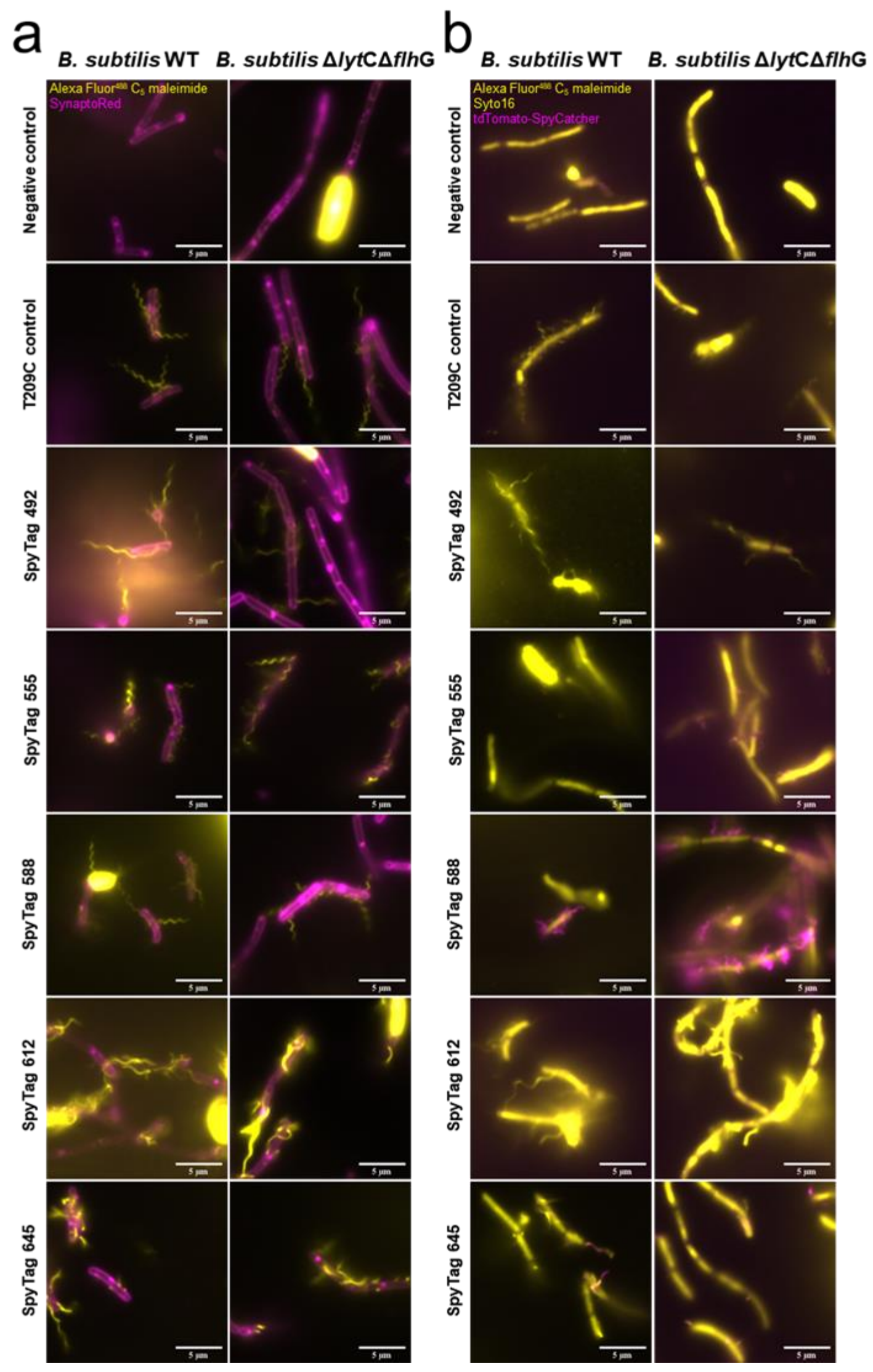


Fig. S9: Characterization of phenotypes and functions of SpyTag-modified flagella.

$B$. subtilis WT and $B$. subtilis $\triangle / y t C \Delta f / h G$ strains were transformed with pRBBm34$\mathrm{Hag}^{\mathrm{T} 209 \mathrm{C}}:$ :SpyT\#\# (dye reactive Cys, SpyTag insertions at indicated nucleotide locations) plasmids and flagella phenotypes and functions compared to strains transformed with pRBBm34$\mathrm{Hag}^{\mathrm{T} 209 \mathrm{C}}$ and $\mathrm{pCT}$-empty plasmid as positive and negative controls, respectively.

a Fluorescence microscopy to evaluate flagella morphologies due to SpyTag insertion. Individual colonies from plates were inoculated in $4 \mathrm{~mL}$ LB medium (supplemented with tetracycline) and grown overnight at $30^{\circ} \mathrm{C}$ and $220 \mathrm{rpm}$. The hag gene is expressed from its native promoter. Flagella were stained with Alexa Fluor ${ }^{\mathrm{TM}} 488 \mathrm{C}_{5}$ maleimide and cell membranes with SynaptoRed ${ }^{\mathrm{TM}}$ C2. Samples were examined using a Nikon Eclipse 90i microscope at 100X. dsRed (excitation 530-560 nm, emission 590-650 nm) and GFP (excitation 450-490 nm, emission 500-550 nm) channels were captured in black and white. Channels were false colored magenta for RFP and yellow for GFP and merged using FIJI.

b Fluorescence microscopy to evaluate SpyTag function when inserted at different locations of the Hag protein. Cultures were grown as above but with the addition of $0.15 \mathrm{mg}$ purified tdTomatoSpyCatcher protein to label functionally displayed SpyTags on flagella. Flagella were stained with Alexa Fluor ${ }^{\mathrm{TM}} 488 \mathrm{C}_{5}$ maleimide and cells visualized with the nucleic acid stain Syto ${ }^{\mathrm{TM}} 16$. Samples were imaged and channels false colored as above. 
a

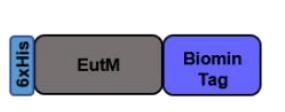

CotB: SGRARAQRSSRGR R5: SSKKGSYSGSKGSKRRIL SB7: RQSSRGR

Synthetic: KFFEAAAKKFFE

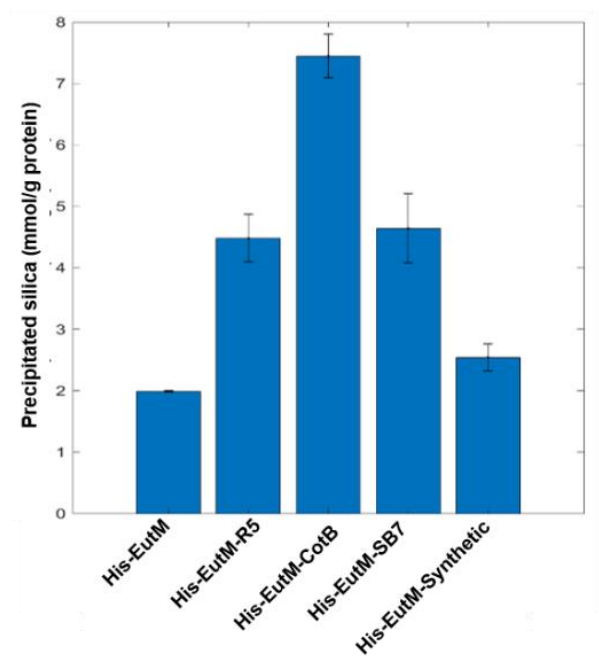

b
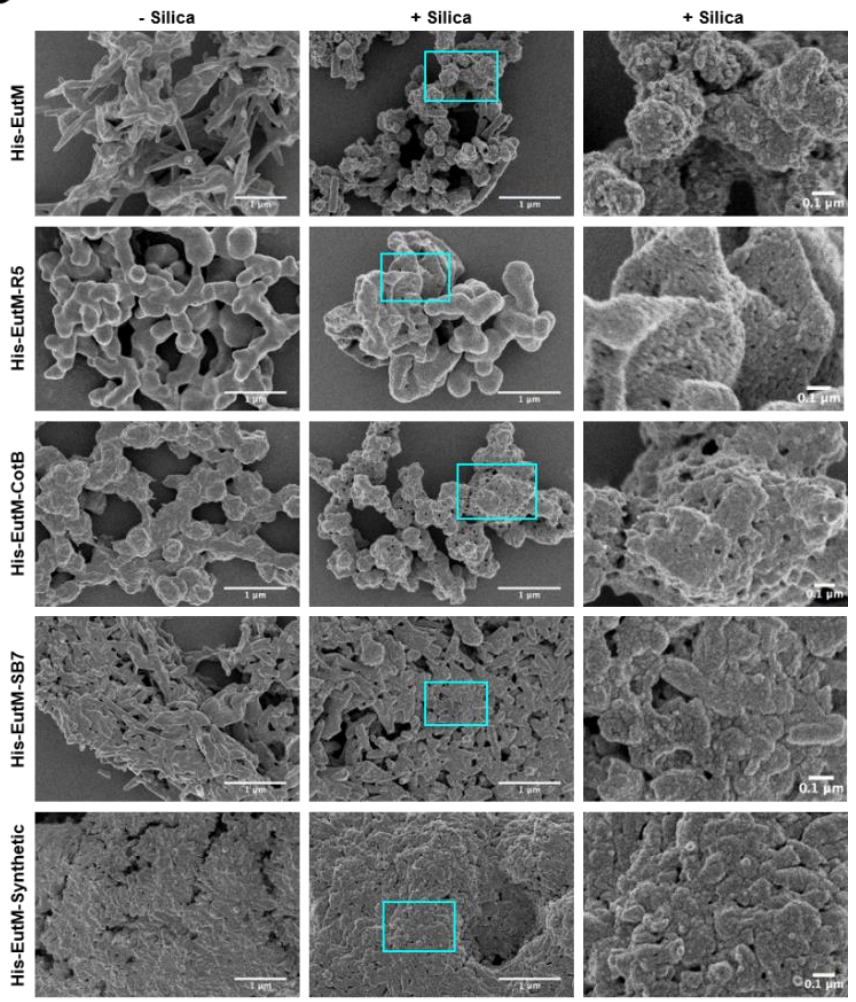

Fig. S10: Characterization of silica binding and precipitation by EutM biomineralization peptide fusion proteins.

a Four silica binding peptides were fused to His-EutM (top panel) and silica binding to the purified proteins $(1 \mathrm{mg} / \mathrm{mL}$ protein incubated with $100 \mathrm{mM}$ silica for $2 \mathrm{~h}$ ) compared to His-EutM. Silica precipitation was quantified spectrophotometrically with the molybdate blue assay. Error bars represent standard deviations calculated for values from three sample replicates.

b The same protein scaffolds $(1 \mathrm{mg} / \mathrm{mL}$ ) incubated without (- silica; left column) and with (+ silica $(100 \mathrm{mM})$; middle, and right) for $2 \mathrm{~h}$ were visualized by SEM. Deposition of silica increases surface roughness and porosity, and also forms silica nanoparticle aggregates on the surface. His-EutMCotB shows the strongest silica precipitation. His-EutM also precipitates silica due to its His-tag and positive charge ${ }^{3-6}$. 

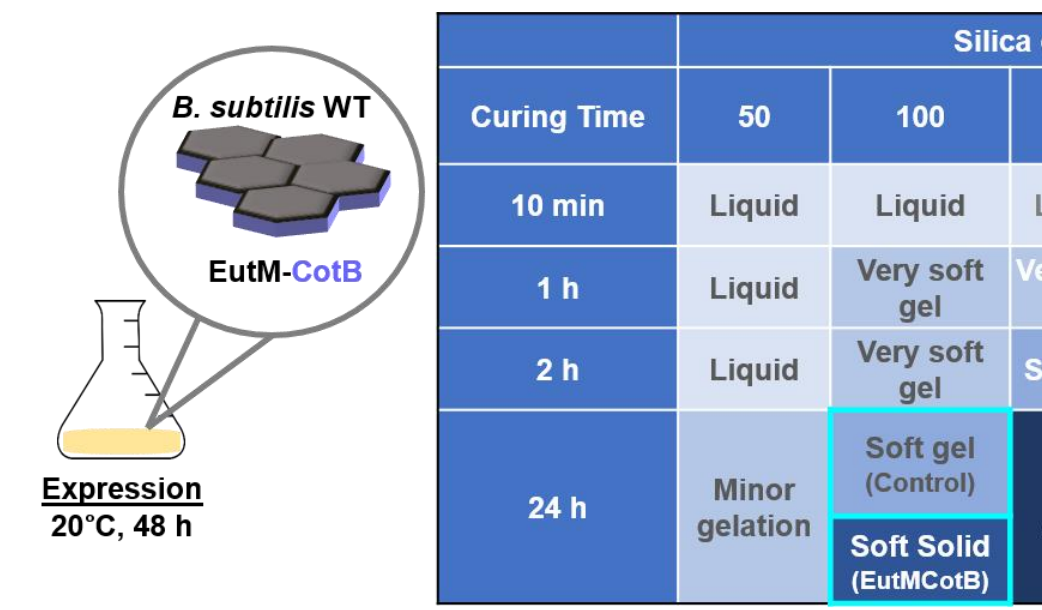

ilica concentration $(\mathrm{mM})$
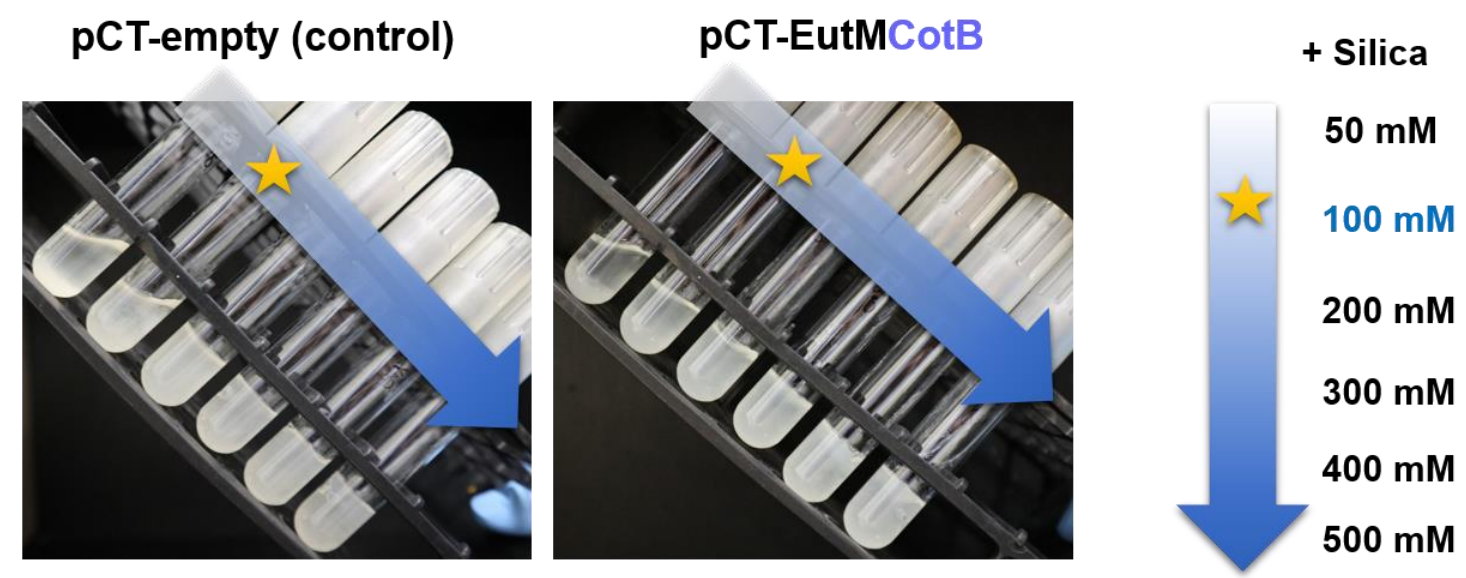

Fig. S11: Comparison and optimization of silica gel formation by B. subtilis WT cultures expressing EutM-CotB scaffolds or no scaffolds.

B. subtilis WT pCT-empty (control) or pCT-EutMCotB cultures were grown in SMM at $20^{\circ} \mathrm{C}$ for 48 $\mathrm{h}$ after induction of protein expression. Different silica concentrations $(50-500 \mathrm{mM})$ were then added to $2.5 \mathrm{~mL}$ culture samples and silica gel formation followed for $24 \mathrm{~h}$ at $20^{\circ} \mathrm{C}$. The addition of $100 \mathrm{mM}$ silica created a soft solid silica gel after $24 \mathrm{~h}$ with EutM-CotB expressing culture samples while control culture samples did not yield a solidified gel. Lower silica concentration did not form a gel and higher concentrations rapidly formed a hard solid, regardless of culture sample (top panel). Bottom panel shows the solidification of these samples with increasing silica concentrations added (left to right). Cultures expressing EutM-CotB solidified with $100 \mathrm{mM}$ silica (labeled with a star) while the control culture sample remained liquid with $100 \mathrm{mM}$ silica. 

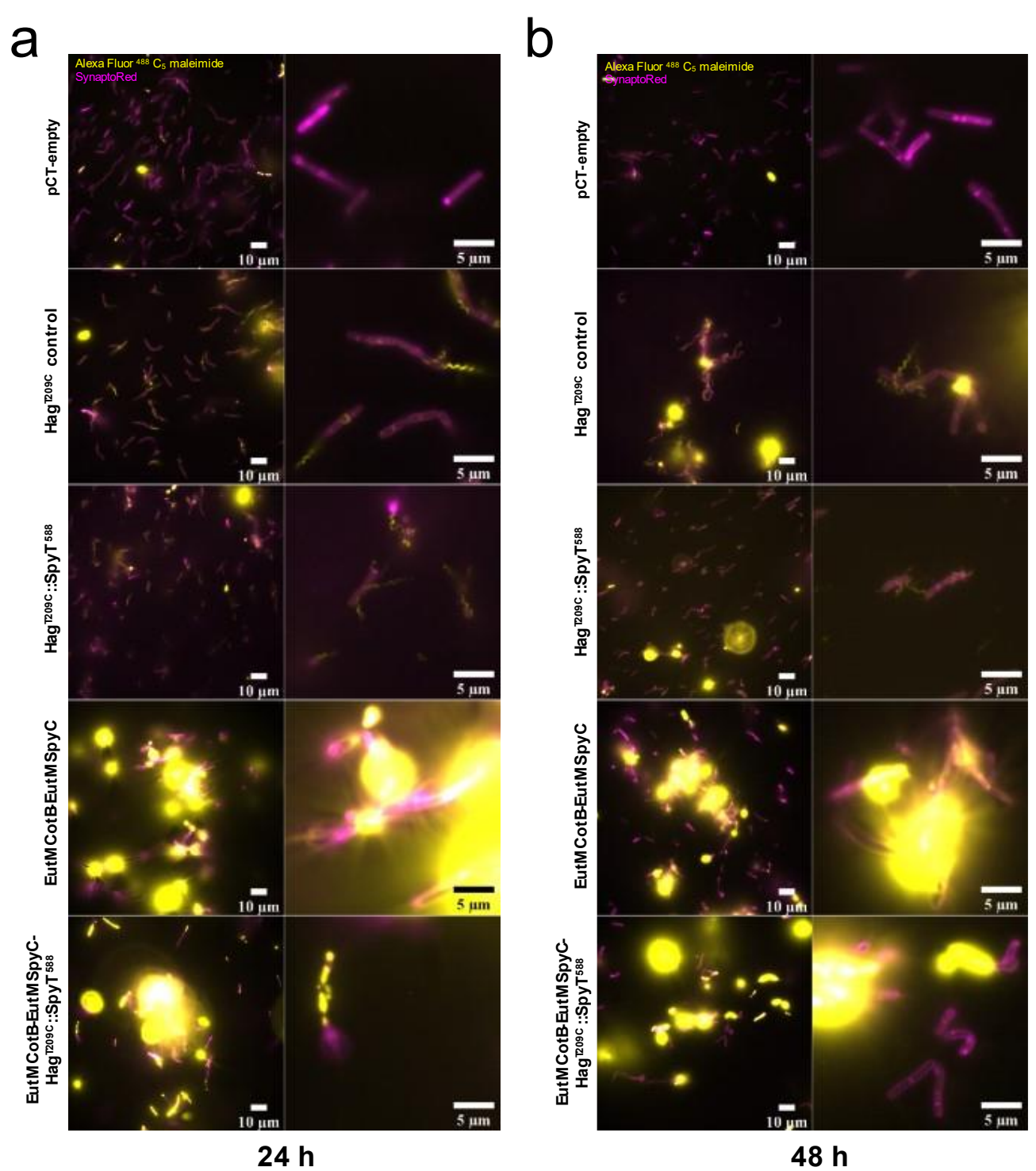

\section{Fig. S12: Visualization of cell phenotypes of $B$. subtilis $\Delta / y t C \Delta$ flhG cultures transformed with different plasmids.}

B. subtilis $\Delta / y t C \Delta f / h G$ was transformed with plasmids for the expression of modified flagella (pRBBm34-Hag ${ }^{\text {T209C }}$ and pRBBm34-Hag ${ }^{T 209 C}: . S p y T^{588}$ ), EutM scaffold building blocks (pCTEutMCotB-EutMSpyC and pCT-empty(control)), and SpyTagged flagella and EutM scaffold building blocks (pCT-EutMCotB-EutMSpyC-Hag ${ }^{T 209 C}$ ::SpyT ${ }^{588}$ ). Strains harboring the pCT plasmids were grown for up to $48 \mathrm{~h}$ in $\mathrm{SMM}$ at $20^{\circ} \mathrm{C}$ under induction condition to express EutM scaffolds. Strains harboring the pRBBm34 plasmids were grown under the same conditions, but without induction. After 24 (a) and $48 \mathrm{~h}$ (b), samples were stained with the cysteine reactive dye Alexa Fluor ${ }^{\mathrm{TM}} 488 \mathrm{C}_{5}$ maleimide and the cell membrane dye SynaptoRed ${ }^{\mathrm{TM}} \mathrm{C} 2$. Samples from EutM scaffold building block expressing cultures contained secreted scaffolds that appeared to be stained with the cysteine reactive Alexa Fluor dye, presumably due to labeling of one or both Cys residues present in the EutM protein. 


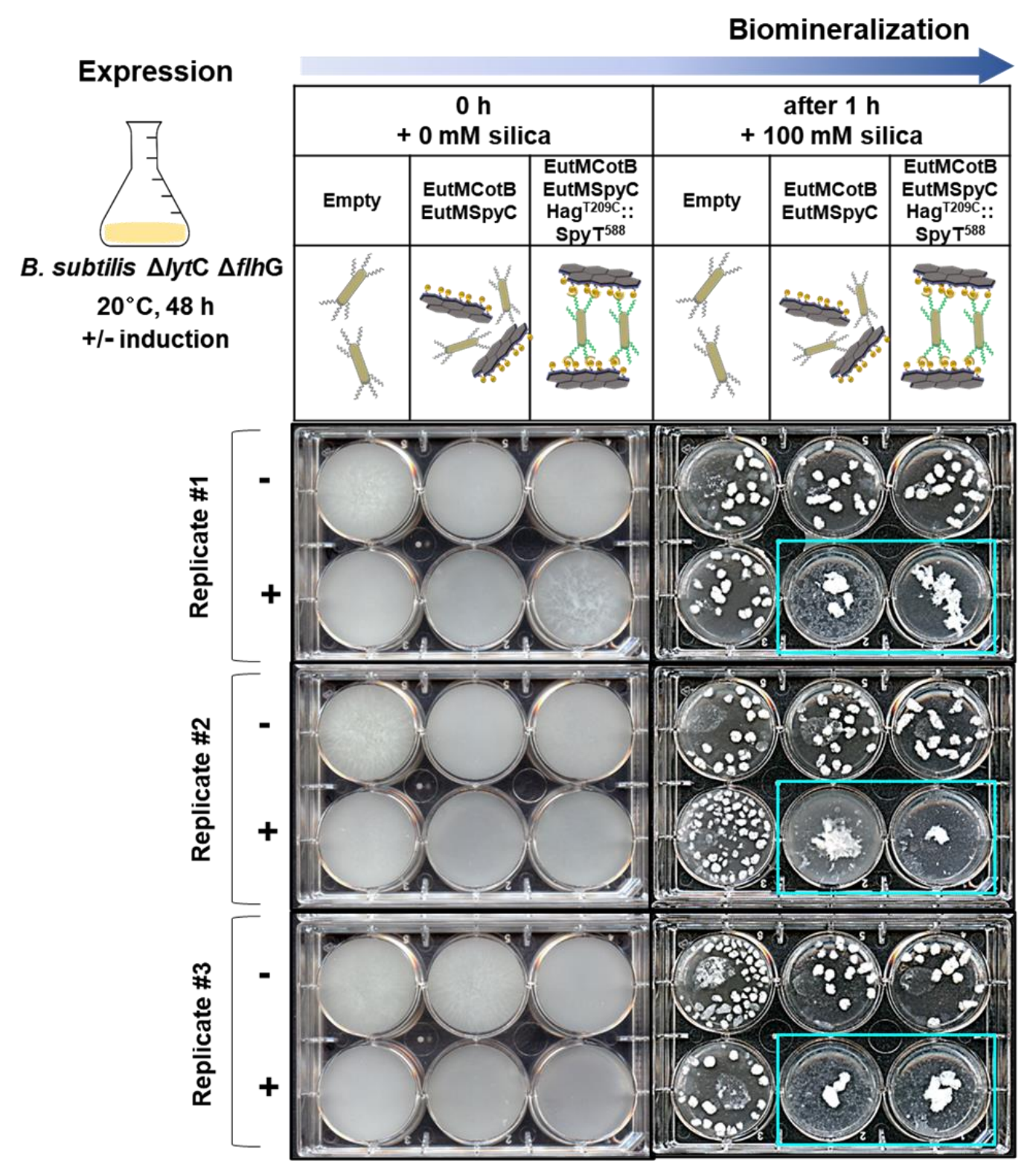

Fig. S13: Silica biomineralization by engineered $B$. subtilis $\Delta / y t C \Delta f / h G$ cultures.

B. subtilis $\triangle l y t C \triangle f l h G$ strains harboring three different pCT plasmids (second row) were grown in $\mathrm{SMM}$ at $20^{\circ} \mathrm{C}$ for $48 \mathrm{~h}$ under uninduced (-) and induced (+) conditions for protein expression. 5 $\mathrm{mL}$ cultures were then transferred to 6-well plates for silica biomineralization with $100 \mathrm{mM}$ silica at $20^{\circ} \mathrm{C}, 100 \mathrm{rpm}$ for $1 \mathrm{~h}$. Experiments were performed with three biological culture replicates that are shown. Only cultures that co-expressed EutM-CotB and EutM-SpyCatcher formed aggregated silica materials upon induction. The aggregation appears to be more pronounced in cultures that also co-expressed SpyTag labeled flagella for cross-linking of cells and EutM scaffolds. 


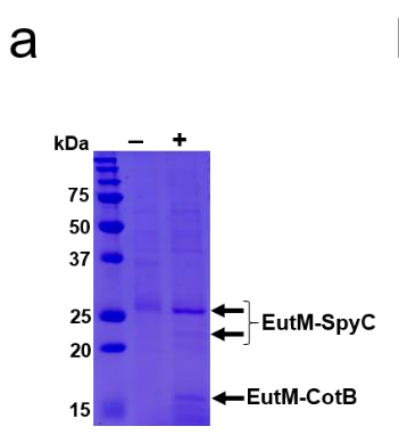

$(-)$ pCT-empty (+) pCT-EutMCotB-EutMSpyC-Hag ${ }^{\text {T209C::Spy }}$ T $^{588}$ b

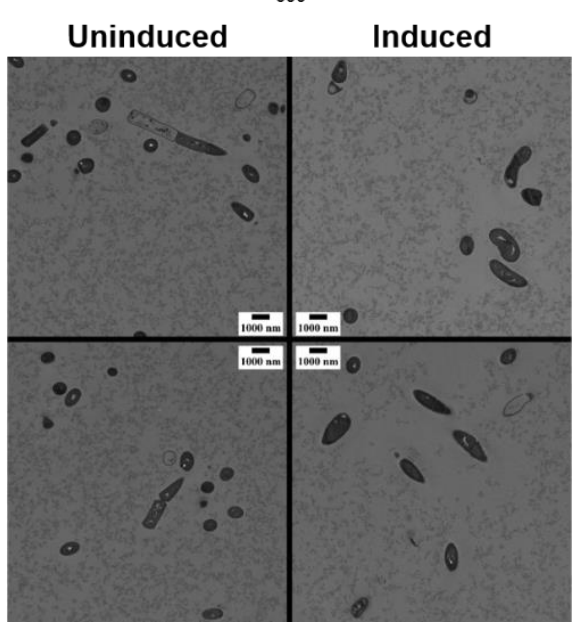

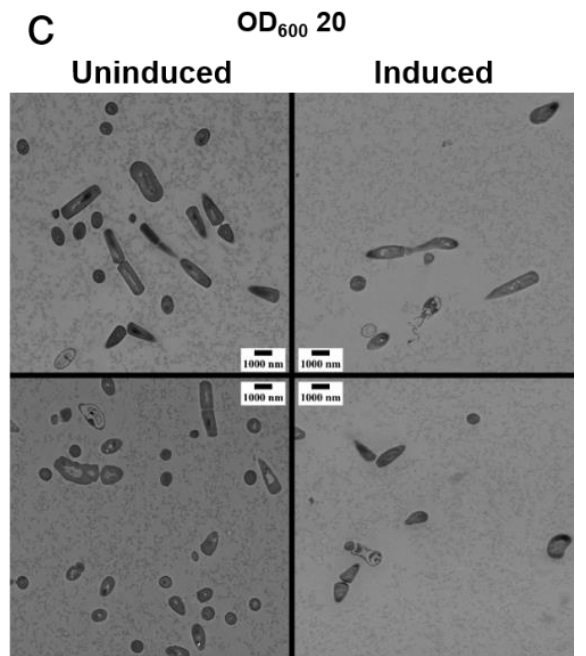

Fig. S14: Additional characterization of Bacillus silica biocomposites.

a Silica gel blocks from $1 \mathrm{~mL}$ cultures (see Fig. $\mathbf{6 b}$ ) were dissolved in SDS-PAGE loading buffer to confirm the presence of EutM scaffold proteins in the silica blocks by SDS-PAGE. Silica blocks made from empty vector control (-) cultures show no scaffold proteins while silica blocks from the EutMCotB-EutMSpyC-Hag ${ }^{\top 209 C}: . S p y T^{588}$ induced $(+)$ cultures have bands with the expected sizes for EutM-SpyCatcher and EutM-CotB.

b, c Silica blocks generated from concentrated cultures with cell densities adjusted to $\mathrm{OD}_{600}$ of 10 or 20 were viewed by TEM for comparison to silica gel blocks directly formed from cultures (Fig. 7). B. subtilis $\Delta / y t C \Delta f / h G$ pCT-EutMCotB-EutMSpyC-Hag ${ }^{T 209 C}:: S p y T^{588}$ was first grown under inducing (induced) or non-inducing (uninduced) conditions for protein expression (SMM, $20^{\circ} \mathrm{C}, 48$ h). Cultures were centrifuged to collect secreted scaffolds and cells and resuspended in collected SMM supernatants to OD 10 (b) or 20 (c). Silica gel blocks were then formed by adding $200 \mathrm{mM}$ silica and $1 \mathrm{~mL}$ aliquots of the cultures were transferred to syringes as molds for curing at $25^{\circ} \mathrm{C}$. After $5 \mathrm{~h}$ of curing at $25^{\circ} \mathrm{C}$, solid gel plugs were cut into $2 \mathrm{~mm}^{3}$ pieces and prepared for thin sectioning. Cells were observed in clusters for both the uninduced and induced cultures, however only the induced culture contained clear zones around cells as was observed with unconcentrated cultures (Fig. 7). 


\section{SUPPORTING TABLES}

Table S1: Plasmids and strains used in this study.

\begin{tabular}{|c|c|c|}
\hline Plasmid & Description & Source \\
\hline \multicolumn{3}{|c|}{ E. coli expression plasmids } \\
\hline pCT5-His-EutM & $\begin{array}{l}\text { eutM (NP_461400) containing a N-terminal } 6 x H i s \text { tag } \\
\text { fused via a GS-linker to generate N-His-GS-EutM, } P_{c t 5} \\
\left.\text { promoter, Amp }{ }^{R} \text { (originally named: } p C T 5 B B-E u t M\right)\end{array}$ & 1,2 \\
\hline pCT5-His-EutMSpyC & $\begin{array}{l}\text { derived from pCT5-His-EutM: EutM shell protein with N- } \\
\text { terminal His tag and C-terminal SpyCatcher }\end{array}$ & 1,2 \\
\hline pCT5-His-EutMCotB & $\begin{array}{l}\text { derived from pCT5-His-EutM: EutM shell protein with N- } \\
\text { terminal His tag and C-terminal CotB silica binding tag }\end{array}$ & This study \\
\hline pCT5-His-EutMR5 & $\begin{array}{l}\text { derived from pCT5-His-EutM: EutM shell protein with } \mathrm{N} \text { - } \\
\text { terminal His tag and C-terminal R5 silica binding tag }\end{array}$ & This study \\
\hline pCT5-His-EutMSB7 & $\begin{array}{l}\text { derived from pCT5-His-EutM: EutM shell protein with N- } \\
\text { terminal His tag and C-terminal SB7 silica binding tag }\end{array}$ & This study \\
\hline pCT5-His-EutMSynthetic & $\begin{array}{l}\text { derived from pCT5-His-EutM: EutM shell protein with } \mathrm{N} \text { - } \\
\text { terminal His tag and C-terminal Synthetic silica binding tag }\end{array}$ & This study \\
\hline tdTomato-pBAD & His-tagged tdTomato cloned into pBAD expression vector & 12 \\
\hline & & $\begin{array}{l}\text { Addgene } \\
\# 54856\end{array}$ \\
\hline pSB1C3-tsPurple & Constitutive expression of purple chromoprotein & $\begin{array}{l}13 \\
\text { Addgene } \\
\# 117848\end{array}$ \\
\hline pET28a-eGFP & $\begin{array}{l}\text { N-terminal } 6 x \text { His tag followed by a thrombin cleavage site } \\
\text { fused to GFP, T7 promoter, } \mathrm{Km}^{\mathrm{R}}\end{array}$ & 2 \\
\hline pET28a-SpyTag-eGFP & $\begin{array}{l}\text { N-terminal } 6 x \text { His tag followed by a thrombin cleavage site } \\
\text { and GS linker fused to SpyTag-eGFP, T7 promoter, } \mathrm{Km}^{\mathrm{R}}\end{array}$ & 2 \\
\hline \multicolumn{3}{|c|}{ B. subtilis expression plasmids } \\
\hline pCT5-bac2.0 & $\begin{array}{l}\text { Designed for cumate inducible gene expression in Bacilli, } \\
\text { PxylR-CymR and } P_{\text {Ст5-CuO-sfGFP, } \text { Amp }^{R}, \text { Tet }^{R}}\end{array}$ & $\begin{array}{l}11 \\
\text { Addgene } \\
\# 119872\end{array}$ \\
\hline pCT-empty & sfGFP deleted from pCT5-bac2.0 & This study \\
\hline pCT-EutMSpyC & $\begin{array}{l}\text { Derived from pCT5-bac2.0 by replacing sfGFP with } \\
\text { levansucrase signal peptide (SacB), N-terminal His tag, } \\
\text { EutM and C-terminal SpyCatcher }\end{array}$ & This study \\
\hline
\end{tabular}




\begin{tabular}{|c|c|c|}
\hline Plasmid & Description & Source \\
\hline pCT-XynAEutMSpyC & $\begin{array}{l}\text { Derived from pCT5-bac2.0 by replacing sfGFP with } \\
\text { xylanase A signal peptide (XynA), N-terminal His tag, } \\
\text { EutM and C-terminal SpyCatcher }\end{array}$ & This study \\
\hline pCT-YngKEutMSpyC & $\begin{array}{l}\text { Derived from pCT5-bac2.0 by replacing sfGFP with fusion } \\
\text { of glycosyl hydrolase signal peptide (YngK), N-terminal His } \\
\text { tag, EutM and C-terminal SpyCatcher }\end{array}$ & This study \\
\hline pCT-CelAEutMSpyC & $\begin{array}{l}\text { Derived from pCT5-bac2.0 by replacing sfGFP with } \\
\text { Cellulase A signal peptide (CelA), N-terminal His tag, EutM } \\
\text { and C-terminal SpyCatcher }\end{array}$ & This study \\
\hline pCT-LipAEutMSpyC & $\begin{array}{l}\text { Derived from pCT5-bac2.0 by replacing sfGFP with lipase } \\
\text { A signal peptide (LipA), N-terminal His tag, EutM and C- } \\
\text { terminal SpyCatcher domain }\end{array}$ & This study \\
\hline pCT-EutMCotB & $\begin{array}{l}\text { Derived from pCT5-bac2.0 by replacing sfGFP with } \\
\text { levansucrase signal peptide (SacB), N-terminal His tag, } \\
\text { EutM and C-terminal CotB tag }\end{array}$ & This study \\
\hline pCT-EutMCotB-EutMSpyC & $\begin{array}{l}\text { Hybrid plasmid of SacB-His-EutM-CotB and SacB-His- } \\
\text { EutM-SpyC modules, each gene expressed from a } \\
\text { pCTbac2.0 cumate inducible promoter }\end{array}$ & This study \\
\hline pCT-EutMCotB-EutMSpyC-Hag ${ }^{\top 209 C:: S p y T^{588}}$ & 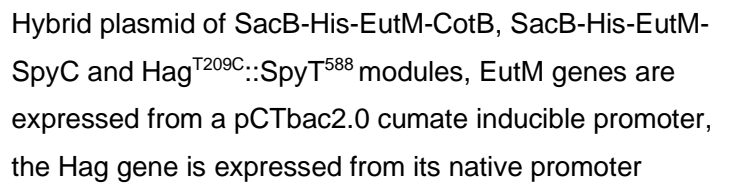 & This study \\
\hline pCT-His-tdTomatoSpyC & $\begin{array}{l}\text { Fluorescent tdTomato with SpyCatcher expressed from } \\
\text { cumated inducible promoter }\end{array}$ & This study \\
\hline pCT-Purple-Hag ${ }^{\text {T209C.::SpyT }}{ }^{588}$ & $\begin{array}{l}\text { Purple chromoprotein expression cassette combined with } \\
\text { Hag expression cassette, chromoprotein expression from } \\
\text { pCTbac2.0 cumate inducible promoter, Hag gene } \\
\text { expression from native promoter }\end{array}$ & This study \\
\hline pRBBm34 & $\begin{array}{l}\text { Bacillus expression vector, GFP expression under control } \\
\text { of native xylose inducible promoter of } B \text {. megaterium, } \\
\text { Amp }^{R}, \text { Tet }^{R}\end{array}$ & $\begin{array}{l}21 \\
\text { Addgene } \\
\# 48114\end{array}$ \\
\hline pRBBm34-Hag ${ }^{\text {T209C }}$ & $\begin{array}{l}\text { hag gene from B. subtilis WT (with T209C mutation in Hag } \\
\text { protein) under control of native hag promoter (NP) }\end{array}$ & This study \\
\hline pRBBm34-Hag ${ }^{\top 209 C .: S p y T^{399}}$ & $\begin{array}{l}\text { hagT209C with SpyTag insertion after } 399 \text { nucleotides } \\
\text { (counting from start codon) under control of native hag } \\
\text { promoter (NP) }\end{array}$ & This study \\
\hline pRBBm34-Hag ${ }^{\top 209 C .: S p y T^{426}}$ & $\begin{array}{l}\text { hagT209C with SpyTag insertion after } 426 \text { nucleotides } \\
\text { under control of native hag promoter (NP) }\end{array}$ & This study \\
\hline
\end{tabular}




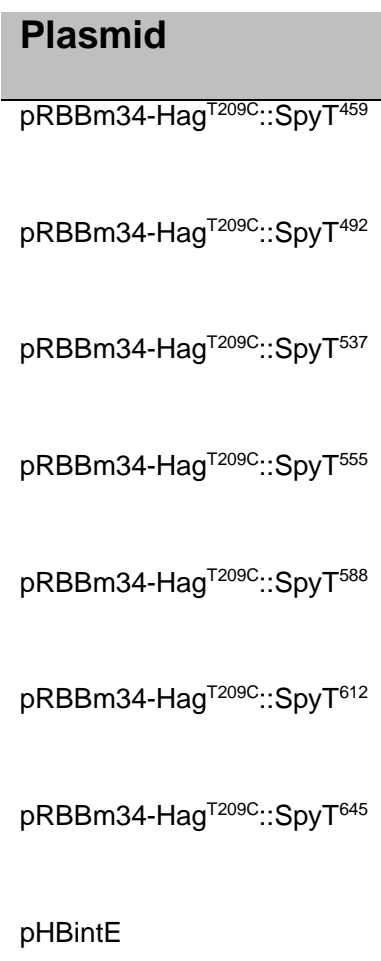

\section{Description}

hagT209C with SpyTag insertion after 459 nucleotides

under control of native hag promoter (NP)

hagT209C with SpyTag insertion after 492 nucleotides

under control of native hag promoter (NP)

hagT209C with SpyTag insertion after 537 nucleotides

under control of native hag promoter (NP)

hagT209C with SpyTag insertion after 555 nucleotides

under control of native hag promoter (NP)

hagT209C with SpyTag insertion after 588 nucleotides

under native promoter

hagT209C with SpyTag insertion after 612 nucleotides

under control of native hag promoter (NP)

hagT209C with SpyTag insertion after 645 nucleotides

under control of native hag promoter (NP)

Cloning, expression and integration vector for Bacillus spp. with xylose-inducible promoter PxylA; Amp ${ }^{R}$, orits

lytC upstream and downstream sequences cloned into pHBintE vector for in frame deletion of lytC

flhG upstream and downstream sequences cloned into pHBintE vector for in frame deletion of flhG
Addgene

\#48136

This study

\section{Source}

This study

This study

This study

This study

This study

This study

This study

22

This study

\begin{tabular}{|c|c|c|}
\hline Strain & Description & Source \\
\hline Escherichia coli Top10 & General cloning host & Invitrogen \\
\hline \multirow[t]{2}{*}{ Escherichia coli C2566 } & Protein expression & New England \\
\hline & & Biolabs \\
\hline Bacillus subtilis WT & Bacillus subtilis wild-type strain 168 & ATCC 23857 \\
\hline Bacillus subtilis $\Delta / y t C$ & B. subtilis WT engineered to retain the endospore & This study \\
\hline Bacillus subtilis $\Delta l y t C \Delta f l h G$ & $\begin{array}{l}\text { B. subtilis WT engineered to have polar flagella and to retain the } \\
\text { endospore }\end{array}$ & This study \\
\hline
\end{tabular}


Table S2: Amino acid sequences of proteins and peptides in this study.

\begin{tabular}{|c|c|}
\hline Name & Amino acid sequences \\
\hline SacB & MNIKKFAKQATVLTFTTALLAGGATQAFAAST \\
\hline EutM & $\begin{array}{l}\text { EALGMIETRGLVALIEASDAMVKAARVKLVGVKQIGGGLCTAMVRGDVAACKAATDAGAAAAQRIGELVSV } \\
\text { HVIPRPHGDLEEVFPISFKGDSNIVDGSGGSGG }\end{array}$ \\
\hline SpyCatcher & $\begin{array}{l}\text { DSATHIKFSKRDEDGKELAGATMELRDSSGKTISTWISDGQVKDFYLYPGKYTFVETAAPDGYEVATAITFT } \\
\text { VNEQGQVTVNG }\end{array}$ \\
\hline SpyTag & AHIVMVDAYKPTK \\
\hline CotB & SGRARAQRQSSRGR \\
\hline R5 & SSKKSGSYSGSKGSKRRIL \\
\hline SB7 & RQSSRGR \\
\hline Synthetic & KFFEAAAKKFFE \\
\hline His-EutM & $\begin{array}{l}\text { MHHHHHHGSGSGSGSGSGSEALGMIETRGLVALIEASDAMVKAARVKLVGVKQIGGGLCTAMVRGDVAA } \\
\text { CKAATDAGAAAAQRIGELVSVHVIPRPHGDLEEVFPISFKGDSNI }\end{array}$ \\
\hline His-EutM-CotB & $\begin{array}{l}\text { MHHHHHHGSGSGSGSGSGSEALGMIETRGLVALIEASDAMVKAARVKLVGVKQIGGGLCTAMVRGDVAA } \\
\text { CKAATDAGAAAAQRIGELVSVHVIPRPHGDLEEVFPISFKGDSNIVDGSGGSGGSGRARAQRQSSRGR }\end{array}$ \\
\hline His-EutM-R5 & $\begin{array}{l}\text { MHHHHHHGSGSGSGSGSGSEALGMIETRGLVALIEASDAMVKAARVKLVGVKQIGGGLCTAMVRGDVAA } \\
\text { CKAATDAGAAAAQRIGELVSVHVIPRPHGDLEEVFPISFKGDSNIVDGSGGSGGSSKKSGSYSGSKGSKR } \\
\text { RIL }\end{array}$ \\
\hline His-EutM-SB7 & $\begin{array}{l}\text { MHHHHHHGSGSGSGSGSGSEALGMIETRGLVALIEASDAMVKAARVKLVGVKQIGGGLCTAMVRGDVAA } \\
\text { CKAATDAGAAAAQRIGELVSVHVIPRPHGDLEEVFPISFKGDSNIVDGSGGSGGRQSSRGR }\end{array}$ \\
\hline $\begin{array}{l}\text { His-EutM- } \\
\text { Synthetic }\end{array}$ & $\begin{array}{l}\text { MHHHHHHGSGSGSGSGSGSEALGMIETRGLVALIEASDAMVKAARVKLVGVKQIGGGLCTAMVRGDVAA } \\
\text { CKAATDAGAAAAQRIGELVSVHVIPRPHGDLEEVFPISFKGDSNIVDGSGGSGGKFFEAAAKKFFE }\end{array}$ \\
\hline $\begin{array}{l}\text { SacB-His-EutM- } \\
\text { SpyCatcher }\end{array}$ & $\begin{array}{l}\text { MNIKKFAKQATVLTFTTALLAGGATQAFAASTHHHHHHGSGSGSGSGSGSEALGMIETRGLVALIEASDAM } \\
\text { VKAARVKLVGVKQIGGGLCTAMVRGDVAACKAATDAGAAAAQRIGELVSVHVIPRPHGDLEEVFPISFKGD } \\
\text { SNIVDGSGGSGGDSATHIKFSKRDEDGKELAGATMELRDSSGKTISTWISDGQVKDFYLYPGKYTFVETAA } \\
\text { PDGYEVATAITFTVNEQGQVTVNG }\end{array}$ \\
\hline $\begin{array}{l}\text { SacB-His-EutM- } \\
\text { CotB }\end{array}$ & $\begin{array}{l}\text { MNIKKFAKQATVLTFTTALLAGGATQAFAASTHHHHHHGSGSGSGSGSGSEALGMIETRGLVALIEASDAM } \\
\text { VKAARVKLVGVKQIGGGLCTAMVRGDVAACKAATDAGAAAAQRIGELVSVHVIPRPHGDLEEVFPISFKGD } \\
\text { SNIVDGSGGSGGSGRARAQRQSSRGR }\end{array}$ \\
\hline $\mathrm{Hag}^{\mathrm{T} 209 \mathrm{C}}$ & $\begin{array}{l}\text { MRINHNIAALNTLNRLSSNNSASQKNMEKLSSGLRINRAGDDAAGLAISEKMRGQIRGLEMASKNSQDGIS } \\
\text { LIQTAEGALTETHAILQRVRELVVQAGNTGTQDKATDLQSIQDEISALTDEIDGISNRTEFNGKKLLDGTYKV } \\
\text { DTATPANQKNLVFQIGANATQQISVNIEDMGADALGIKEADGSIAALHSVNDLDVTKFADNAADCADIGFDA } \\
\text { QLKVVDEAINQVSSQRAKLGAVQNRLEHTINNLSASGENLTAAESRIRDVDMAKEMSEFTKNNILSQASQA } \\
\text { MLAQANQQPQNVLQLLR }\end{array}$ \\
\hline
\end{tabular}




\begin{tabular}{|c|c|}
\hline Name & Imino acid sequences \\
\hline c.:Spy $T^{399}$ & $\begin{array}{l}\text { MRINHNIAALNTLNRLSSNNSASQKNMEKLSSGLRINRAGDDAAGLAISEKMRGQIRGLEMASKNSQDGIS } \\
\text { LIQTAEGALTETHAILQRVRELVVQAGNTGTQDKATDLQSIQDEISALTDEIDGISNRTEFNAHIVMVDAYKP } \\
\text { TKGKKLLDGTYKVDTATPANQKNLVFQIGANATQQISVNIEDMGADALGIKEADGSIAALHSVNDLDVTKFA } \\
\text { DNAADCADIGFDAQLKVVDEAINQVSSQRAKLGAVQNRLEHTINNLSASGENLTAAESRIRDVDMAKEMSE } \\
\text { FTKNNILSQASQAMLAQANQQPQNVLQLLR }\end{array}$ \\
\hline c.:SpyT ${ }^{426}$ & $\begin{array}{l}\text { MRINHNIAALNTLNRLSSNNSASQKNMEKLSSGLRINRAGDDAAGLAISEKMRGQIRGLEMASKNSQDGIS } \\
\text { LIQTAEGALTETHAILQRVRELVVQAGNTGTQDKATDLQSIQDEISALTDEIDGISNRTEFNGKKLLDGTYAHII } \\
\text { VIMVDAYKPTKKVDTATPANQKNLVFQIGANATQQISVNIEDMGADALGIKEADGSIAALHSVNDLDVTKFAD } \\
\text { NAADCADIGFDAQLKVVDEAINQVSSQRAKLGAVQNRLEHTINNLSASGENLTAAESRIRDVDMAKEMSEF } \\
\text { TKNNILSQASQAMLAQANQQPQNVLQLLR }\end{array}$ \\
\hline 9C.:.Spy $T^{459}$ & $\begin{array}{l}\text { MRINHNIAALNTLNRLSSNNSASQKNMEKLSSGLRINRAGDDAAGLAISEKMRGQIRGLEMASKNSQDGIS } \\
\text { LIQTAEGALTETHAILQRVRELVVQAGNTGTQDKATDLQSIQDEISALTDEIDGISNRTEFNGKKLLDGTYKV } \\
\text { DTATPANQKAHIVMVDAYKPTKNLVFQIGANATQQISVNIEDMGADALGIKEADGSIAALHSVNDLDVTKFA } \\
\text { DNAADCADIGFDAQLKVVDEAINQVSSQRAKLGAVQNRLEHTINNLSASGENLTAAESRIRDVDMAKEMSE } \\
\text { FTKNNILSQASQAMLAQANQQPQNVLQLLR }\end{array}$ \\
\hline $\mathrm{Hag}$ & $\begin{array}{l}\text { MRINHNIAALNTLNRLSSNNSASQKNMEKLSSGLRINRAGDDAAGLAISEKMRGQIRGLEMASKNSQDGIS } \\
\text { LIQTAEGALTETHAILQRVRELVVQAGNTGTQDKATDLQSIQDEISALTDEIDGISNRTEFNGKKLLDGTYKV } \\
\text { DTATPANQKNLVFQIGANATAHIVMVDAYKPTKQQISVNIEDMGADALGIKEADGSIAALHSVNDLDVTKFA } \\
\text { DNAADCADIGFDAQLKVVDEAINQVSSQRAKLGAVQNRLEHTINNLSASGENLTAAESRIRDVDMAKEMSE } \\
\text { FTKNNILSQASQAMLAQANQQPQNVLQLLR }\end{array}$ \\
\hline $\mathrm{Hag}^{\mathrm{T} 209 \mathrm{C}}:: \mathrm{Spy} \top^{537}$ & $\begin{array}{l}\text { MRINHNIAALNTLNRLSSNNSASQKNMEKLSSGLRINRAGDDAAGLAISEKMRGQIRGLEMASKNSQDGIS } \\
\text { LIQTAEGALTETHAILQRVRELVVQAGNTGTQDKATDLQSIQDEISALTDEIDGISNRTEFNGKKLLDGTYKV } \\
\text { DTATPANQKNLVFQIGANATQQISVNIEDMGADALAHIVMVDAYKPTKGIKEADGSIAALHSVNDLDVTKFA } \\
\text { DNAADCADIGFDAQLKVVDEAINQVSSQRAKLGAVQNRLEHTINNLSASGENLTAAESRIRDVDMAKEMSE } \\
\text { FTKNNILSQASQAMLAQANQQPQNVLQLLR }\end{array}$ \\
\hline $\mathrm{Hag}^{\mathrm{T} 209 \mathrm{C}}:: \mathrm{Spy} T^{555}$ & $\begin{array}{l}\text { MRINHNIAALNTLNRLSSNNSASQKNMEKLSSGLRINRAGDDAAGLAISEKMRGQIRGLEMASKNSQDGIS } \\
\text { LIQTAEGALTETHAILQRVRELVVQAGNTGTQDKATDLQSIQDEISALTDEIDGISNRTEFNGKKLLDGTYKV } \\
\text { DTATPANQKNLVFQIGANATQQISVNIEDMGADALGIKEADAHIVMVDAYKPTKGSIAALHSVNDLDVTKFA } \\
\text { DNAADCADIGFDAQLKVVDEAINQVSSQRAKLGAVQNRLEHTINNLSASGENLTAAESRIRDVDMAKEMSE } \\
\text { FTKNNILSQASQAMLAQANQQPQNVLQLLR }\end{array}$ \\
\hline $\mathrm{Hag}^{\mathrm{T} 209 \mathrm{C}}:: \mathrm{Spy} T^{588}$ & $\begin{array}{l}\text { MRINHNIAALNTLNRLSSNNSASQKNMEKLSSGLRINRAGDDAAGLAISEKMRGQIRGLEMASKNSQDGIS } \\
\text { LIQTAEGALTETHAILQRVRELVVQAGNTGTQDKATDLQSIQDEISALTDEIDGISNRTEFNGKKLLDGTYKV } \\
\text { DTATPANQKNLVFQIGANATQQISVNIEDMGADALGIKEADGSIAALHSVNDAHIVMVDAYKPTKLDVTKFA } \\
\text { DNAADCADIGFDAQLKVVDEAINQVSSQRAKLGAVQNRLEHTINNLSASGENLTAAESRIRDVDMAKEMSE } \\
\text { FTKNNILSQASQAMLAQANQQPQNVLQLLR }\end{array}$ \\
\hline $\mathrm{Hag}^{\text {T209C}}:: \mathrm{Spy}^{612}$ & $\begin{array}{l}\text { MRINHNIAALNTLNRLSSNNSASQKNMEKLSSGLRINRAGDDAAGLAISEKMRGQIRGLEMASKNSQDGIS } \\
\text { LIQTAEGALTETHAILQRVRELVVQAGNTGTQDKATDLQSIQDEISALTDEIDGISNRTEFNGKKLLDGTYKV } \\
\text { DTATPANQKNLVFQIGANATQQISVNIEDMGADALGIKEADGSIAALHSVNDLDVTKFADAHIVMVDAYKPT } \\
\text { KNAADCADIGFDAQLKVVDEAINQVSSQRAKLGAVQNRLEHTINNLSASGENLTAAESRIRDVDMAKEMSE } \\
\text { FTKNNILSQASQAMLAQANQQPQNVLQLLR }\end{array}$ \\
\hline
\end{tabular}




\begin{tabular}{|c|c|}
\hline Name & Amino acid sequences \\
\hline $\operatorname{Hag}^{\text {T209C.::SpyT }}{ }^{645}$ & $\begin{array}{l}\text { MRINHNIAALNTLNRLSSNNSASQKNMEKLSSGLRINRAGDDAAGLAISEKMRGQIRGLEMASKNSQDGIS } \\
\text { LIQTAEGALTETHAILQRVRELVVQAGNTGTQDKATDLQSIQDEISALTDEIDGISNRTEFNGKKLLDGTYKV } \\
\text { DTATPANQKNLVFQIGANATQQISVNIEDMGADALGIKEADGSIAALHSVNDLDVTKFADNAADCADIGFDA } \\
\text { HIVMVDAYKPTKAQLKVVDEAINQVSSQRAKLGAVQNRLEHTINNLSASGENLTAAESRIRDVDMAKEMSE } \\
\text { FTKNNILSQASQAMLAQANQQPQNVLQLLR }\end{array}$ \\
\hline & $\begin{array}{l}\text { MHHHHHHGSGSGSGSGSGSVDVSKGEEVIKEFMRFKVRMEGSMNGHEFEIEGEGEGRPYEGTQTAKLK } \\
\text { VTKGGPLPFAWDILSPQFMYGSKAYVKHPADIPDYKKLSFPEGFKWERVMNFEDGGLVTVTQDSSLQDGT } \\
\text { LIYKVKMRGTNFPPDGPVMQKKTMGWEASTERLYPRDGVLKGEIHQALKLKDGGHYLVEFKTIYMAKKPV } \\
\text { QLPGYYYVDTKLDITSHNEDYTIVEQYERSEGRHHLFLGHGTGSTGSGSSGTASSEDNNMAVIKEFMRFK } \\
\text { VRMEGSMNGHEFEIEGEGEGRPYEGTQTAKLKVTKGGPLPFAWDILSPQFMYGSKAYVKHPADIPDYKKL } \\
\text { SFPEGFKWERVMNFEDGGLVTVTQDSSLQDGTLIYKVKMRGTNFPPDGPVMQKKTMGWEASTERLYPR } \\
\text { DGVLKGEIHQALKLKDGGHYLVEFKTIYMAKKPVQLPGYYYVDTKLDITSHNEDYTIVEQYERSEGRHHLFL } \\
\text { YGMDELYKGSGGSGGDSATHIKFSKRDEDGKELAGATMELRDSSGKTISTWISDGQVKDFYLYPGKYTFV } \\
\text { ETAAPDGYEVATAITFTVNEQGQVTVNG }\end{array}$ \\
\hline 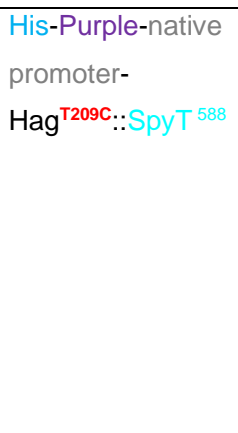 & $\begin{array}{l}\text { MHHHHHHSSGLVPRGSMASLVKKDMCVKMTMEGTVNGYHFKCVGEGEGKPFEGTQNMRIRVTEGGPLP } \\
\text { FAFDILAPCCMYGSKTFIKHVSGIPDYFKESFPEGFTWERTQIFEDGGVLTAHQDTSLEGNCLIYKVKVLGT } \\
\text { NFPANGPVMQKKTAGWEPCVEMLYPRDGVLCGQSLMALKCTDGNHLTSHLRTTYRSRKPSNAVNMPEF } \\
\text { HFGDHRIEILKAEQGKFYEQYESAVARYSDVPEKAT [untranslated region] } \\
\text { MRINHNIAALNTLNRLSSNNSASQKNMEKLSSGLRINRAGDDAAGLAISEKMRGQIRGLEMASKNSQDGIS } \\
\text { LIQTAEGALTETHAILQRVRELVVAGNTGTQDKATDLQSIQDEISALTDEIDGISNRTEFNGKKLLDGTYKV } \\
\text { DTATPANQKNLVFQIGANATQQISVNIEDMGADALGIKEADGSIAALHSVNDAHIVMVDAYKPTKLDVTKFA } \\
\text { DNAADCADIGFDAQLKVVDEAINQVSSQRAKLGAVQNRLEHTINNLSASGENLTAAESRIRDVDMAKEMSE } \\
\text { FTKNNILSQASQAMLAQANQQPQNVLQLLR }\end{array}$ \\
\hline
\end{tabular}


Table S3: Nucleotides sequences of proteins and peptides in this study.

\begin{tabular}{|c|c|}
\hline Name & Nucleotide sequence \\
\hline \multirow[t]{5}{*}{ EutM } & GAAGCATTAGGAATGATTGAAACCCGGGGCCTGGTTGCGCTGATTGAGGCCTCCGATGCGATGGTAA \\
\hline & AAGCCGCGCGCGTGAAGCTGGTCGGCGTGAAGCAGATTGGCGGTGGCCTGTGTACTGCCATGGTGC \\
\hline & GTGGCGATGTGGCGGCGTGCAAAGCCGCAACCGATGCTGGCGCCGCTGCGGCGCAGCGCATTGGC \\
\hline & GAGTTGGTCTCCGTACACGTGATTCCACGCCCGCACGGCGATCTGGAAGAAGTGTTCCCGATCAGCT \\
\hline & TCAAAGGCGACAGCAACATT \\
\hline \multirow[t]{4}{*}{ SpyCatcher } & GATAGTGCTACCCATATTAAATTCTCAAAACGTGATGAGGACGGCAAAGAGTTAGCTGGTGCAACTAT \\
\hline & GGAGTTGCGTGATTCATCTGGTAAAACTATTAGTACATGGATTTCAGATGGACAAGTGAAAGATTTCTA \\
\hline & CCTGTATCCAGGAAAATATACATTTGTCGAAACCGCAGCACCAGACGGTTATGAGGTAGCAACTGCTA \\
\hline & TTACCTTTACAGTTAATGAGCAAGGTCAGGTTACTGTAAATGGC \\
\hline SpyTag & GCCCACATCGTGATGGTGGACGCCTACAAGCCGACGAAG \\
\hline CotB & AGCGGAGGCAGCGGCCGTGCGCGAGCTCAGCGTCAAAGCTCACGTGGGCGC \\
\hline R5 & AGCTCTAAGAAAAGTGGCAGCTATTCAGGCAGCAAAGGTTCGAAGCGACGCATTCTG \\
\hline SB7 & CGTCAAAGCTCACGTGGGCGC \\
\hline Synthetic & AAGTTCTTTGAAGCTGCCGCGAAGAAATTCTTTGAA \\
\hline \multirow[t]{6}{*}{ His-EutM } & ATGCATCATCATCACCACCACGGTTCTGGTTCTGGTTCTGGTTCTGGTTCTGGTTCTGAAGCATTAGGA \\
\hline & ATGATTGAAACCCGGGGCCTGGTTGCGCTGATTGAGGCCTCCGATGCGATGGTAAAAGCCGCGCGC \\
\hline & GTGAAGCTGGTCGGCGTGAAGCAGATTGGCGGTGGCCTGTGTACTGCCATGGTGCGTGGCGATGTG \\
\hline & GCGGCGTGCAAAGCCGCAACCGATGCTGGCGCCGCTGCGGCGCAGCGCATTGGCGAGTTGGTCTCC \\
\hline & GTACACGTGATTCCACGCCCGCACGGCGATCTGGAAGAAGTGTTCCCGATCAGCTTCAAAGGCGACA \\
\hline & GCAACATT \\
\hline \multirow[t]{7}{*}{ His-EutM-CotB } & ATGCATCATCATCACCACCACGGTTCTGGTTCTGGTTCTGGTTCTGGTTCTGGTTCTGAAGCATTAGGA \\
\hline & ATGATTGAAACCCGGGGCCTGGTTGCGCTGATTGAGGCCTCCGATGCGATGGTAAAAGCCGCGCGC \\
\hline & GTGAAGCTGGTCGGCGTGAAGCAGATTGGCGGTGGCCTGTGTACTGCCATGGTGCGTGGCGATGTG \\
\hline & GCGGCGTGCAAAGCCGCAACCGATGCTGGCGCCGCTGCGGCGCAGCGCATTGGCGAGTTGGTCTCC \\
\hline & GTACACGTGATTCCACGCCCGCACGGCGATCTGGAAGAAGTGTTCCCGATCAGCTTCAAAGGCGACA \\
\hline & GCAACATTGTCGACGGGAGTGGTGGCAGCGGAGGCAGCGGAGGCAGCGGCCGTGCGCGAGCTCAG \\
\hline & CGTCAAAGCTCACGTGGGCGC \\
\hline \multirow[t]{7}{*}{ His-EutM-R5 } & ATGCATCATCATCACCACCACGGTTCTGGTTCTGGTTCTGGTTCTGGTTCTGGTTCTGAAGCATTAGGA \\
\hline & ATGATTGAAACCCGGGGCCTGGTTGCGCTGATTGAGGCCTCCGATGCGATGGTAAAAGCCGCGCGC \\
\hline & GTGAAGCTGGTCGGCGTGAAGCAGATTGGCGGTGGCCTGTGTACTGCCATGGTGCGTGGCGATGTG \\
\hline & GCGGCGTGCAAAGCCGCAACCGATGCTGGCGCCGCTGCGGCGCAGCGCATTGGCGAGTTGGTCTCC \\
\hline & GTACACGTGATTCCACGCCCGCACGGCGATCTGGAAGAAGTGTTCCCGATCAGCTTCAAAGGCGACA \\
\hline & GCAACATTGTCGACGGGAGTGGTGGCAGCGGAGGCAGCTCTAAGAAAAGTGGCAGCTATTCAGGCA \\
\hline & GCAAAGGTTCGAAGCGACGCATTCTG \\
\hline \multirow[t]{3}{*}{ His-EutM-SB7 } & ATGCATCATCATCACCACCACGGTTCTGGTTCTGGTTCTGGTTCTGGTTCTGGTTCTGAAGCATTAGGA \\
\hline & ATGATTGAAACCCGGGGCCTGGTTGCGCTGATTGAGGCCTCCGATGCGATGGTAAAAGCCGCGCGC \\
\hline & GTGAAGCTGGTCGGCGTGAAGCAGATTGGCGGTGGCCTGTGTACTGCCATGGTGCGTGGCGATGTG \\
\hline
\end{tabular}




\begin{tabular}{ll} 
Name & Nucleotide sequence \\
& GCGGCGTGCAAAGCCGCAACCATGCTGGCGCCGCTGCGGCGCAGCGCATTGGCGAGTTGGTCTCC \\
& GTACACGTGATTCCACGCCCGCACGGCGATCTGGAAGAATGTTCCCGATCAGCTTCAAAGGCGACA \\
& GCAACATTGTCGACGGGAGTGTGGCAGCGGAGGCCGTCAAAGCTCACGTGGGCGC \\
\hline His-EutM- & ATGCATCATCATCACCACCACGGTTCTGGTTCTGGTTCTGGTTCTGGTTCTGGTTCTGAAGCATTAGGA \\
ATGATTGAAACCCGGGGCCTGGTTGCGCTGATTGAGGCCTCCGATGCGATGGTAAAACCGCGCGC & GTGAAGCTGGTCGGCGTGAAGCAGATTGGCGGTGGCCTGTGTACTGCCATGGTGCGTGGCGATGTG \\
& GCGGCGTGCAAAGCCGCAACCGATGCTGGCGCCGCTGCGGCGCAGCGCATTGGCGAGTTGGTCTCC \\
& GTACACGTGATTCCACGCCCGCACGGCGATCTGGAAGAAGTGTTCCCGATCAGCTTCAAAGGCGACA \\
& GCAACATTGTCGACGGGAGTGGTGGCAGCGGAGGCAAGTTCTTTGAAGCTGCCGCGAAGAAATCTT \\
& TGAA
\end{tabular}

SacB-His-EutM- $\quad$ ATGAACATCAAAAAGTTTGCAAAACAAGCAACAGTATTAACCTTTACTACCGCACTGCTGGCAGGAGG SpyCatcher CGCAACTCAAGCGTTTGCGGCTTCAACTCATCATCATCACCACCACGGTTCTGGTTCTGGTTCTGGTT CTGGTTCTGGTTCTGAAGCATTAGGAATGATTGAAACCCGGGGCCTGGTTGCGCTGATTGAGGCCTC CGATGCGATGGTAAAAGCCGCGCGCGTGAAGCTGGTCGGCGTGAAGCAGATTGGCGGTGGCCTGTG TACTGCCATGGTGCGTGGCGATGTGGCGGCGTGCAAAGCCGCAACCGATGCTGGCGCCGCTGCGGC GCAGCGCATTGGCGAGTTGGTCTCCGTACACGTGATTCCACGCCCGCACGGCGATCTGGAAGAAGTG TTCCCGATCAGCTTCAAAGGCGACAGCAACATTGTCGACGGGAGTGGTGGCAGCGGAGGCGATAGTG CTACCCATATTAAATTCTCAAAACGTGATGAGGACGGCAAAGAGTTAGCTGGTGCAACTATGGAGTTG CGTGATTCATCTGGTAAAACTATTAGTACATGGATTTCAGATGGACAAGTGAAAGATTTCTACCTGTAT CCAGGAAAATATACATTTGTCGAAACCGCAGCACCAGACGGTTATGAGGTAGCAACTGCTATTACCTTT ACAGTTAATGAGCAAGGTCAGGTTACTGTAAATGGCTAA

SacB-His-EutM- $\quad$ ATGAACATCAAAAAGTTTGCAAAACAAGCAACAGTATTAACCTTTACTACCGCACTGCTGGCAGGAGG CotB CGCAACTCAAGCGTTTGCGGCTTCAACTCATCATCATCACCACCACGGTTCTGGTTCTGGTTCTGGTT CTGGTTCTGGTTCTGAAGCATTAGGAATGATTGAAACCCGGGGCCTGGTTGCGCTGATTGAGGCCTC CGATGCGATGGTAAAAGCCGCGCGCGTGAAGCTGGTCGGCGTGAAGCAGATTGGCGGTGGCCTGTG TACTGCCATGGTGCGTGGCGATGTGGCGGCGTGCAAAGCCGCAACCGATGCTGGCGCCGCTGCGGC GCAGCGCATTGGCGAGTTGGTCTCCGTACACGTGATTCCACGCCCGCACGGCGATCTGGAAGAAGTG TTCCCGATCAGCTTCAAAGGCGACAGCAACATTGTCGACGGGAGTGGTGGCAGCGGAGGCAGCGGC CGTGCGCGAGCTCAGCGTCAAAGCTCACGTGGGCGCTGA

native hag promoter $\operatorname{Hag}^{\top 209 C .: S p y \top}$ 588
AACTAGTGTGCAGCAGCGTTATCCAGCGATGTGATCTCCGCATTATCCTCACAAAAAAAGTGAGGATTT TTTTATTTTTGTATTAACAAAATCAGAGACAATCCGATATTAATGATGTAGCCGGGAGGAGGCGCAAAA GACTCAGCCAGTTACAAAATAAGGGCACAAGGACGTGCCTTAACAACATATTCAGGGAGGAACAAAAC AATGAGAATTAACCACAATATTGCAGCGCTTAACACACTGAACCGTTTGTCTTCAAACAACAGTGCGAG CCAAAAGAACATGGAGAAACTTTCTTCAGGTCTTCGCATCAACCGTGCGGGAGATGACGCAGCAGGT CTTGCGATCTCTGAAAAATGAGAGGACAAATCAGAGGTCTTGAAATGGCTTCTAAAAACTCTCAAGAC GGAATCTCTCTTATCCAAACAGCTGAGGGTGCATTAACTGAAACTCATGCGATCCTTCAACGTGTTCGT GAGCTAGTTGTTCAAGCTGGAAACACTGGAACTCAGGACAAAGCAACTGATTTGCAATCTATTCAAGAT GAAATTTCAGCTTTAACAGATGAAATCGATGGTATTTCAAATCGTACAGAATTCAATGGTAAGAAATTGC TCGATGGCACTTACAAAGTTGACACAGCTACTCCTGCAAATCAAAAGAACTTGGTATTCCAAATCGGAG CAAATGCTACACAGCAAATCTCTGTAAATATTGAGGATATGGGTGCTGACGCTCTTGGAATTAAAGAAG CTGATGGTTCAATTGCAGCTCTTCATTCAGTTAATGATGCCCACATCGTGATGGTGGACGCCTACAAG CCGACGAAGCTTGACGTAACAAAATTCGCAGATAATGCAGCAGATTGTGCTGATATCGGTTTCGATGC TCAATTGAAAGTTGTTGATGAAGCGATCAACCAAGTTTCTTCTCAACGTGCTAAGCTTGGTGCGGTACA 


\section{Name Nucleotide sequence \\ AAATCGTCTAGAGCACACAATTAACAACTTAAGCGCTTCTGGTGAAAACTTGACAGCTGCTGAGTCTCG TATCCGTGACGTTGACATGGCTAAAGAGATGAGCGAATTCACAAAGAACAACATTCTTTCTCAGGCTTC TCAAGCTATGCTTGCTCAAGCAAACCAACAGCCGCAAAACGTACTTCAATTATTACGTTAA}

His-tdTomatoSpyCatcher
ATGCATCATCATCACCACCACGGTTCTGGTTCTGGTTCTGGTTCTGGTTCTGGTTCTGTCGACGTGAG CAAGGGCGAGGAGGTCATCAAAGAGTTCATGCGCTTCAAGGTGCGCATGGAGGGCTCCATGAACGG CCACGAGTTCGAGATCGAGGGCGAGGGCGAGGGCCGCCCCTACGAGGGCACCCAGACCGCCAAGC TGAAGGTGACCAAGGGCGGCCCCCTGCCCTTCGCCTGGGACATCCTGTCCCCCCAGTTCATGTACGG CTCCAAGGCGTACGTGAAGCACCCCGCCGACATCCCCGATTACAAGAAGCTGTCCTTCCCCGAGGGC TTCAAGTGGGAGCGCGTGATGAACTTCGAGGACGGCGGTCTGGTGACCGTGACCCAGGACTCCTCC CTGCAGGACGGCACGCTGATCTACAAGGTGAAGATGCGCGGCACCAACTTCCCCCCCGACGGCCCC GTAATGCAGAAGAAGACCATGGGCTGGGAGGCCTCCACCGAGCGCCTGTACCCCCGCGACGGCGTG CTGAAGGGCGAGATCCACCAGGCCCTGAAGCTGAAGGACGGCGGCCACTACCTGGTGGAGTTCAAG ACCATCTACATGGCCAAGAAGCCCGTGCAACTGCCCGGCTACTACTACGTGGACACCAAGCTGGACA TCACCTCCCACAACGAGGACTACACCATCGTGGAACAGTACGAGCGCTCCGAGGGCCGCCACCACCT GTTCCTGGGGCATGGCACCGGCAGCACCGGCAGCGGCAGCTCCGGCACCGCCTCCTCCGAGGACAA CAACATGGCCGTCATCAAAGAGTTCATGCGCTTCAAGGTGCGCATGGAGGGCTCCATGAACGGCCAC GAGTTCGAGATCGAGGGCGAGGGCGAGGGCCGCCCCTACGAGGGCACCCAGACCGCCAAGCTGAA GGTGACCAAGGGCGGCCCCCTGCCCTTCGCCTGGGACATCCTGTCCCCCCAGTTCATGTACGGCTCC AAGGCGTACGTGAAGCACCCCGCCGACATCCCCGATTACAAGAAGCTGTCCTTCCCCGAGGGCTTCA AGTGGGAGCGCGTGATGAACTTCGAGGACGGCGGTCTGGTGACCGTGACCCAGGACTCCTCCCTGC AGGACGGCACGCTGATCTACAAGGTGAAGATGCGCGGCACCAACTTCCCCCCCGACGGCCCCGTAAT GCAGAAGAAGACCATGGGCTGGGAGGCCTCCACCGAGCGCCTGTACCCCCGCGACGGCGTGCTGAA GGGCGAGATCCACCAGGCCCTGAAGCTGAAGGACGGCGGCCACTACCTGGTGGAGTTCAAGACCAT CTACATGGCCAAGAAGCCCGTGCAACTGCCCGGCTACTACTACGTGGACACCAAGCTGGACATCACC TCCCACAACGAGGACTACACCATCGTGGAACAGTACGAGCGCTCCGAGGGCCGCCACCACCTGTTCC TGTACGGCATGGACGAGCTGTACAAGGGGAGTGGTGGCAGCGGAGGCGATAGTGCTACCCATATTAA ATTCTCAAAACGTGATGAGGACGGCAAAGAGTTAGCTGGTGCAACTATGGAGTTGCGTGATTCATCTG GTAAAACTATTAGTACATGGATTTCAGATGGACAAGTGAAAGATTTCTACCTGTATCCAGGAAAATATA CATTTGTCGAAACCGCAGCACCAGACGGTTATGAGGTAGCAACTGCTATTACCTTTACAGTTAATGAG CAAGGTCAGGTTACTGTAAATGGCTGA

His-PurpleATGCATCATCATCATCATCACAGCAGCGGCCTGGTGCCGCGCGGCAGCATGGCGAGCTTGGTTAAGA native promoterAAGATATGTGTGTTAAGATGACGATGGAAGGTACTGTGAACGGTTATCACTTTAAGTGCGTTGGCGAG

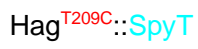
588 GGTGAAGGCAAGCCGTTCGAGGGCACGCAGAACATGCGCATTCGTGTCACCGAGGGCGGTCCGCTG CCTTTTGCATTCGACATCCTGGCCCCGTGCTGTATGTACGGCTCTAAGACCTTCATTAAACACGTGAG CGGTATCCCGGATTACTTTAAAGAGTCCTTTCCAGAGGGCTTCACTTGGGAACGTACCCAGATTTTTGA GGACGGTGGTGTTCTGACCGCGCACCAAGACACCAGCCTGGAAGGTAATTGCCTGATCTATAAAGTG AAGGTTCTGGGTACCAATTTCCCGGCGAATGGTCCGGTGATGCAAAAGAAAACCGCGGGTTGGGAGC CGTGCGTCGAGATGCTGTATCCGCGTGACGGCGTCTTGTGTGGTCAGAGCTTGATGGCGCTGAAGTG CACCGATGGCAATCATCTGACCAGCCACCTGCGCACGACGTATCGTAGCCGTAAACCGAGCAACGCC GTTAACATGCCGGAGTTCCATTTTGGTGACCATCGCATCGAAATCCTGAAAGCTGAGCAGGGCAAATT CTACGAACAATACGAATCGGCTGTCGCACGTTACAGCGATGTGCCGGAAAAAGCGACGTAAGTTAAAA GTCTCGTAAAGCGTTCTATCAATAACCCGTTGGTGCCAGGCATCAAATAAAACGAAAGGCTCAGTCGA 


Name
Nucleotide sequence
AAGACTGGGCCTTTCGTTTTATCTGTTGTTTGTCGGTGAACGCTCTCTACTAGAGAGCTCATGCCGGC
GGCACCTCGCTAACGGATTCACCACTCCAAGAATTGAGCCAGTACAATGGTCCAAACTAGTGTGCAG
CAGCGTTATCCAGCGATGTGATCTCCGCATTATCCTCACAAAAAAATGAGGATTTTTTATTTTGTAT
TAACAAAATCAGAGACAATCCGATATTAATGATGTAGCCGGGAGGAGGCGCAAAAGACTCAGCCAGTT
ACAAAATAAGGGCACAAGGACGTGCCTTAACAACATATTCAGGGAGGAACAAAACAATGAGAATTAAC
CACAATATTGCAGCGCTTAACACACTGAACCGTTTGTCTTCAAACAACAGTGCGAGCCAAAAGAACAT
GGAGAAACTTTCTTCAGGTCTTCGCATCAACCGTGCGGGAGATGACGCAGCAGGTCTTGCGATCTCT
GAAAAAATGAGAGGACAAATCAGAGGTCTTGAAATGGCTTCTAAAAACTCTCAAGACGGAATCTCTCTT
ATCCAAACAGCTGAGGGTGCATTAACTGAAACTCATGCGATCCTTCAACGTGTTCGTGAGCTAGTTGTT
CAAGCTGGAAACACTGGAACTCAGGACAAAGCAACTGATTTGCAATCTATTCAAGATGAAATTTCAGCT
TTAACAGATGAAATCGATGGTATTTCAAATCGTACAGAATTCAATGGTAAGAAATTGCTCGATGGCACT
TACAAAGTTGACACAGCTACTCCTGCAAATCAAAAGAACTTGGTATTCCAAATCGGAGCAAATGCTACA
CAGCAAATCTCTGTAAATATTGAGGATATGGGTGCTGACGCTCTTGGAATTAAAGAAGCTGATGGTTCA
ATTGCAGCTCTTCATTCAGTTAATGATGCCCACATCGTGATGGTGGACGCCTACAAGCCGACGAAGCT
TGACGTAACAAAATTCGCAGATAATGCAGCAGATTGTGCTGATATCGGTTTCGATGCTCAATTGAAAGT
TGTTGATGAAGCGATCAACCAAGTTTCTTCTCAACGTGCTAAGCTTGGTGCGGTACAAAATCGTCTAGA
GCACACAATTAACAACTTAAGCGCTTCTGGTGAAACTTGACAGCTGCTGAGTCTCGTATCCGTGACG
TTGACATGGCTAAAGAGATGAGCGAATTCACAAAGAACAACATTCTTTCTCAGGCTTCTCAAGCTATGC
TTGCTCAAGCAAACCAACAGCCGCAAAACGTACTTCAATTATTACGTTAA


Table S4: Amino acid sequences of signal peptides.

\begin{tabular}{llll}
\hline $\begin{array}{l}\text { Signal } \\
\text { peptides }\end{array}$ & Amino acid sequence & Source & Reference \\
\hline SacB & MNIKKFAKQATVLTFTTALLAGGATQAFAAST & B. subtilis & [Gilbert, 2017] ${ }^{23}$ \\
XynA & MFKFKKNFLVGLSAALMSISLFSATASAAST & B. subtilis & {$\left[\right.$ Gilbert, 2017] ${ }^{23}$} \\
YngK & MYIKKCIGSILFLLLFCSSALPAKAAST & B. megaterium & {$[\text { Stammen, 2010] }]^{24}$} \\
CelA & MKRSISIFITCLLITLLTMGGMIASPASAAST & B. subtilis & {$[\text { [Gilbert, 2017] }]^{23}$} \\
LipA & MKKVLMAFIICLSLILSVLAAPPSGAKAAST & B. megaterium & {$[\text { Stammen, 2010] }]^{24}$} \\
\hline
\end{tabular}




\section{Table S5: List of primers.}

\begin{tabular}{|c|c|}
\hline Name & Sequence \\
\hline PB_Back_fwd & gttaaaagtctcgtaaagcg \\
\hline PB_Back_rev & gtttgtcctccttattagttaatc \\
\hline PB_Ins_fwd & aactaataaggaggacaaacatgcatcatcatcatcatcac \\
\hline PB_Ins_rev & cgctttacgagacttttaacttacgtcgctttttccgg \\
\hline pHBintE seq fwd & tcggaattctcatgtttgacagc \\
\hline pHBintE seq rev & caagggttggtttgcgcatt \\
\hline dlytC_upstream_fwd & tccaaactagaactgaacaatttgtatgattg \\
\hline d/ytC_upstream_rev & aggaaatcaatcgaaagagacaaatctaatc \\
\hline dlytC_downstream_fwd & tctctttcgattgatttcctccttaaatgg \\
\hline dlytC_downstream_rev & tcgaactagtttctacttcaaacagaattagtc \\
\hline dlytC_fwd & tgaagtagaaactagttcgaagatctcc \\
\hline dlytC_rev & ttgttcagttctagtttggaccatttgtc \\
\hline d/ytC_confirmation_fwd & tatcttgcaccatcacccgtc \\
\hline dlytC_confirmation_rev & caagtttatggtggctacagttg \\
\hline dflhG_upstream_fwd & tccaaactagtcgaaaaaaattaaaaagcgaaaatg \\
\hline dflhG_upstream_rev & acgaatcaaaagcatcctcacaaaaccaag \\
\hline dflhG_downstream_fwd & tgaggatgcttttgattcgtgtgcttgtagttg \\
\hline dflhG_downstream_rev & tcgaactagtacccgctttaacagcagc \\
\hline$d f / h G \_f w d$ & taaagcgggtactagttcgaagatctcc \\
\hline dflhG_rev & ttttttcgactagtttggaccatttgtc \\
\hline dflhG_confirmation_fwd & aaagcattggagtggtggat \\
\hline dflhG_confirmation_rev & gaagatcagtcgggtctttctc \\
\hline NP-hag_fwd & caatggtccaaactagtgtgcagcagcg \\
\hline NP-hag_rev & ccggcatgcattaacgtaataattgaagtacgttttgc \\
\hline NP-hag-T209C_fwd & tgcagcagattgtgctgatatcg \\
\hline NP-hag-T209C_rev & ttatctgcgaattttgttac \\
\hline NP-hag-T209C-SPT399_fwd & cgcctacaagccgacgaagggtaagaaattgctcgatg \\
\hline NP-hag-T209C-SPT399_rev & tccaccatcacgatgtgggcattgaattctgtacgatttgaaatac \\
\hline NP-hag-T209C-SPT426_fwd & cgcctacaagccgacgaagaaagttgacacagctactc \\
\hline
\end{tabular}


bioRxiv preprint doi: https://doi.org/10.1101/2021.08.13.456252; this version posted August 13, 2021. The copyright holder for this preprint (which was not certified by peer review) is the author/funder. All rights reserved. No reuse allowed without permission.

\begin{tabular}{|c|c|}
\hline Name & Sequence \\
\hline NP-hag-T209C-SPT426_rev & tccaccatcacgatgtgggcgtaagtgccatcgagcaatttc \\
\hline NP-hag-T209C-SPT459_fwd & cgcctacaagccgacgaagaacttggtattccaaatcg \\
\hline NP-hag-T209C-SPT459_rev & tccaccatcacgatgtgggcctttgatttgcaggagtag \\
\hline NP-hag-T209C-SPT492_fwd & cgcctacaagccgacgaagcagcaaatctctgtaaatattgag \\
\hline NP-hag-T209C-SPT492_rev & tccaccatcacgatgtgggctgtagcatttgctccgatttg \\
\hline NP-hag-T209C-SPT537_fwd & cgcctacaagccgacgaagggaattaaagaagctgatggttcaattg \\
\hline NP-hag-T209C-SPT537_rev & tccaccatcacgatgtgggcaagagcgtcagcacccata \\
\hline NP-hag-T209C-SPT555_fwd & cgcctacaagccgacgaagggttcaattgcagctcttc \\
\hline NP-hag-T209C-SPT555_rev & tccaccatcacgatgtgggcatcagcttctttaattccaag \\
\hline NP-hag-T209C-SPT588_fwd & cgcctacaagccgacgaagcttgacgtaacaaaattcgc \\
\hline NP-hag-T209C-SPT588_rev & tccaccatcacgatgtgggcatcattaactgaatgaagagc \\
\hline NP-hag-T209C-SPT612_fwd & cgcctacaagccgacgaagaatgcagcagattgtgctg \\
\hline NP-hag-T209C-SPT612_rev & tccaccatcacgatgtgggcatctgcgaattttgttacg \\
\hline NP-hag-T209C-SPT645_fwd & cgcctacaagccgacgaaggctcaattgaaagttgttgatgaag \\
\hline NP-hag-T209C-SPT645_rev & tccaccatcacgatgtgggcatcgaaaccgatatcagcac \\
\hline
\end{tabular}


Table S6: Variation of storage modulus (G') and loss modulus (G') with frequency of silica gels.

\begin{tabular}{|c|c|c|c|}
\hline & Storage modulus (G') & & \\
\hline $\begin{array}{l}\text { Angular } \\
\text { frequency } \\
\text { (rad/S) }\end{array}$ & pCT-empty & pCT-EutMCotB-EutMSpyC & 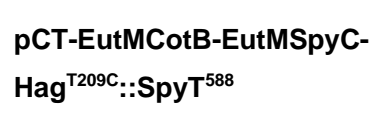 \\
\hline 100 & 1.05.E-03 \pm 2.49.E-04 & 1.29.E-03 \pm 7.29.E-05 & 1.36.E-03 $\pm 2.45 . E-04$ \\
\hline 63.0957 & 1.01.E-03 \pm 2.63.E-04 & 1.21.E-03 \pm 7.88.E-05 & 1.36.E-03 $\pm 2.90 . E-04$ \\
\hline 39.8107 & 9.60.E-04 土 2.52.E-04 & 1.18.E-03 \pm 5.22.E-05 & 1.31.E-03 $\pm 2.45 . E-04$ \\
\hline 25.1189 & 9.38.E-04 — 2.40.E-04 & 1.14.E-03 \pm 4.60.E-05 & 1.27.E-03 $\pm 2.16 . \mathrm{E}-04$ \\
\hline 15.8489 & 9.06.E-04 土 2.29.E-04 & 1.10.E-03 \pm 4.87.E-05 & 1.24.E-03 $\pm 2.04 . E-04$ \\
\hline 10 & 8.84.E-04 土 2.19.E-04 & 1.07.E-03 \pm 4.28.E-05 & 1.20.E-03 \pm 1.77.E-04 \\
\hline 6.30957 & 8.59.E-04 × 2.07.E-04 & 1.02.E-03 \pm 5.88.E-05 & 1.16.E-03 \pm 1.52.E-04 \\
\hline 3.98107 & 8.34.E-04 — 1.59.E-04 & 1.01.E-03 \pm 5.39.E-05 & 1.13.E-03 \pm 1.19.E-04 \\
\hline 2.51189 & 8.32.E-04 — 1.82.E-04 & 9.70.E-04 \pm 5.13.E-05 & 1.11.E-03 \pm 1.31.E-04 \\
\hline 1.58489 & 8.13.E-04 土 1.57.E-04 & 9.11.E-04 \pm 4.23.E-05 & 1.06.E-03 $\pm 1.10 . \mathrm{E}-04$ \\
\hline \multirow[t]{2}{*}{1} & 7.82.E-04 — 1.36.E-04 & 8.92.E-04 \pm 5.50.E-05 & 1.06.E-03 \pm 1.13.E-04 \\
\hline & Loss modulus (G") & & \\
\hline $\begin{array}{l}\text { Angular } \\
\text { frequency } \\
(\mathrm{rad} / \mathrm{S})\end{array}$ & pCT-empty & pCT-EutMCotB-EutMSpyC & $\begin{array}{l}\text { pCT-EutMCotB-EutMSpyC- } \\
\text { Hag }^{\top 209 C:: S p y T}{ }^{588}\end{array}$ \\
\hline 100 & 1.07.E-04 $\pm 2.81 . E-05$ & 9.03.E-05 \pm 5.69.E-05 & 4.37.E-05 \pm 1.23.E-04 \\
\hline 63.0957 & 1.04.E-04 × 6.01.E-05 & 1.12.E-04 \pm 2.32.E-05 & 7.73.E-05 \pm 3.30.E-05 \\
\hline 39.8107 & 1.02.E-04 × 4.18.E-05 & 9.05.E-05 \pm 7.49.E-06 & 7.16.E-05 \pm 1.50.E-05 \\
\hline 25.1189 & 8.00.E-05 \pm 2.92.E-05 & 9.48.E-05 $\pm 2.08 . E-05$ & 7.30.E-05 \pm 1.52.E-05 \\
\hline 15.8489 & 8.52.E-05 — 3.16.E-05 & 9.18.E-05 $\pm 2.48 . E-05$ & 6.45.E-05 $\pm 2.34 . \mathrm{E}-05$ \\
\hline 10 & 8.24.E-05 \pm 2.74.E-05 & 9.03.E-05 \pm 1.17.E-05 & 7.18.E-05 \pm 3.58.E-05 \\
\hline 6.30957 & 9.71.E-05 \pm 3.07.E-05 & 9.46.E-05 $\pm 2.48 . E-05$ & 8.12.E-05 $\pm 2.96 . E-05$ \\
\hline 3.98107 & 9.86.E-05 \pm 2.54.E-05 & 7.06.E-05 \pm 3.59.E-05 & 7.15.E-05 $\pm 2.97 . E-05$ \\
\hline 2.51189 & 9.03.E-05 \pm 1.29.E-05 & 9.40.E-05 \pm 5.46.E-05 & 9.47.E-05 $\pm 2.95 . E-05$ \\
\hline 1.58489 & 8.31.E-05 — 2.09.E-05 & 1.40.E-04 \pm 3.93.E-05 & 9.31.E-05 \pm 1.07.E-05 \\
\hline 1 & 1.27.E-04 — 2.82.E-05 & 1.34.E-04 \pm 3.71.E-05 & 1.09.E-04 $\pm 2.25 . \mathrm{E}-05$ \\
\hline
\end{tabular}




\section{REFERENCES}

1 Schmidt-Dannert, S., Zhang, G., Johnston, T., Quin, M. B. \& Schmidt-Dannert, C. Building a toolbox of protein scaffolds for future immobilization of biocatalysts. Appl Microbiol Biotechnol 102, 8373-8388, doi:10.1007/s00253-018-9252-6 (2018).

2 Zhang, G., Schmidt-Dannert, S., Quin, M. B. \& Schmidt-Dannert, C. Protein-based scaffolds for enzyme immobilization. Methods Enzymol 617, 323-362, doi:10.1016/bs.mie.2018.12.016 (2019).

3 Mieszawska, A. J., Nadkarni, L. D., Perry, C. C. \& Kaplan, D. L. Nanoscale control of silica particle formation via silk-silica fusion proteins for bone regeneration. Chem Mater 22, 5780-5785, doi:10.1021/cm101940u (2010).

4 Groger, C., Lutz, K. \& Brunner, E. Biomolecular self-assembly and its relevance in silica biomineralization. Cell Biochem Biophys 50, 23-39, doi:10.1007/s12013-007-9003-2 (2008).

5 Rodriguez, F., Glawe, D. D., Naik, R. R., Hallinan, K. P. \& Stone, M. O. Study of the chemical and physical influences upon in vitro peptide-mediated silica formation. Biomacromolecules 5, 261-265, doi:10.1021/bm034232c (2004).

6 Zerfass, C., Braukmann, S., Nietzsche, S., Hobe, S. \& Paulsen, H. High yield recombinant production of a self-assembling polycationic peptide for silica biomineralization. Protein Expr Purif 108, 1-8, doi:10.1016/j.pep.2014.12.012 (2015).

7 Anagnostopoulos, C. \& Spizizen, J. Requirements for transformation in Bacillus Subtilis. J Bacteriol 81, 741-746, doi:10.1128/jb.81.5.741-746.1961 (1961).

8 Hauser, P. M. \& Karamata, D. A rapid and simple method for Bacillus subtilis transformation on solid media. Microbiology (Reading) 140 ( Pt 7), 1613-1617, doi:10.1099/13500872-140-7-1613 (1994).

9 Bennallack, P. R., Burt, S. R., Heder, M. J., Robison, R. A. \& Griffitts, J. S. Characterization of a novel plasmid-borne thiopeptide gene cluster in Staphylococcus epidermidis strain 115. J Bacteriol 196, 4344-4350, doi:10.1128/JB.02243-14 (2014).

10 Jamal, M. et al. Ultra-high efficient colony PCR for high throughput screening of bacterial genes. Indian J Microbiol 57, 365-369, doi:10.1007/s12088-017-0665-1 (2017).

11 Seo, S. O. \& Schmidt-Dannert, C. Development of a synthetic cumate-inducible gene expression system for Bacillus. Appl Microbiol Biotechnol 103, 303-313, doi:10.1007/s00253-018-9485-4 (2019).

12 Shaner, N. C. et al. Improved monomeric red, orange and yellow fluorescent proteins derived from Discosoma sp. red fluorescent protein. Nat Biotechnol 22, 1567-1572, doi:10.1038/nbt1037 (2004).

13 Liljeruhm, J. et al. Engineering a palette of eukaryotic chromoproteins for bacterial synthetic biology. J Biol Eng 12, 8, doi:10.1186/s13036-018-0100-0 (2018).

14 Zhang, G. Q., Quin, M. B. \& Schmidt-Dannert, C. Self-assembling protein scaffold system for easy in vitro coimmobilization of biocatalytic cascade enzymes. Acs Catal 8 , 5611-5620, doi:10.1021/acscatal.8b00986 (2018).

15 Zhang, G., Johnston, T., Quin, M. B. \& Schmidt-Dannert, C. Developing a protein scaffolding system for rapid enzyme immobilization and optimization of enzyme functions for biocatalysis. ACS Synth Bio/ 8, 1867-1876, doi:10.1021/acssynbio.9b00187 (2019).

16 Benson, J. J., Sakkos, J. K., Radian, A., Wackett, L. P. \& Aksan, A. Enhanced biodegradation of atrazine by bacteria encapsulated in organically modified silica gels. $J$ Colloid Interf Sci 510, 57-68, doi:10.1016/j.jcis.2017.09.044 (2018).

17 Cha, J. N. et al. Silicatein filaments and subunits from a marine sponge direct the polymerization of silica and silicones in vitro. Proc Natl Acad Sci U S A 96, 361-365, doi:10.1073/pnas.96.2.361 (1999). 
18 Coradin, T., Eglin, D. \& Livage, J. The silicomolybdic acid spectrophotometric method and its application to silicate/biopolymer interaction studies. Spectroscopy-an International Journal 18, 567-576, doi:10.1155/2004/356207 (2004).

19 Sato, T. A modified method for lead staining of thin sections. J Electron Microsc (Tokyo) 17, 158-159, doi:10.1093/oxfordjournals.jmicro.a049610 (1968).

20 Schindelin, J. et al. Fiji: an open-source platform for biological-image analysis. Nat Methods 9, 676-682, doi:10.1038/nmeth.2019 (2012).

21 Biedendieck, R. et al. A sucrose-inducible promoter system for the intra- and extracellular protein production in Bacillus megaterium. J Biotechnol 132, 426-430, doi:10.1016/j.jbiotec.2007.07.494 (2007).

22 Biedendieck, R. et al. Metabolic engineering of cobalamin (vitamin B12) production in Bacillus megaterium. Microb Biotechno/ 3, 24-37, doi:10.1111/j.1751-7915.2009.00125.x (2010).

23 Gilbert, C., Howarth, M., Harwood, C. R. \& Ellis, T. Extracellular self-assembly of functional and tunable protein conjugates from Bacillus subtilis. ACS Synth Bio/6, 957967, doi:10.1021/acssynbio.6b00292 (2017).

24 Stammen, S. et al. High-yield intra- and extracellular protein production using Bacillus megaterium. Appl Environ Microbiol 76, 4037-4046, doi:10.1128/AEM.00431-10 (2010). 